ChipCflow - em hardware dinamicamente reconfigurável

Vitor Fiorotto Astolfi 



\section{ChipCflow - em hardware dinamicamente reconfigurável}

\section{Vitor Fiorotto Astolfi}

Orientador: Prof. Dr. Jorge Luiz e Silva

Dissertação apresentada ao Instituto de Ciências Matemáticas e de Computação - ICMC-USP, como parte dos requisitos para obtenção do título de Mestre em Ciências - Ciências de Computação e Matemática Computacional.

USP - São Carlos

Setembro de 2009 



\section{Dedicatória}

À minha família 


\section{Agradecimentos}

Ao Criador, fonte de inspiração e conforto para a humanidade.

Aos meus pais, Márcia e Márcio, pelo apoio incondicional, pela compreensão, pelo carinho, por possibilitarem a concretização de meus sonhos.

À minha irmã, Érica, e meus irmãos, Fabio e Mauro, pelo companheirismo nos bons e maus momentos, pela constante troca de experiências, pela amizade.

Ao meu orientador, prof. Jorge Luiz e Silva, pelo apoio, dedicação, sobretudo pela paciência com que me conduziu durante todo o tempo, pela confiança e amizade sem as quais não seria possível a conclusão deste trabalho.

Aos professores Ney Laert Vilar Calazans e Marcio Merino Fernandes pelas críticas à monografia do exame de qualificação do mestrado, que facilitou a correção de algumas falhas em meu trabalho.

Aos amigos, colegas e professores da Universidade de São Paulo, em São Carlos, e da Universidade Federal do ABC, pelo companheirismo e pelos bons momentos de convivência, tanto fora quanto dentro da universidade.

Às funcionarias da Seção de Pós-Graduação do ICMC-USP, Ana Paula, Laura, Lívia, e Beth, recentemente aposentada, pela atenção e boa vontade ao atender os alunos e pelos excelentes serviços prestados à comunidade acadêmica.

À Coordenação de Aperfeiçoamento de Pessoal de Nível Superior (CAPES) pelo auxílio financeiro.

E a todos que de maneira direta ou indireta contribuíram para a realização deste trabalho. 


\section{Resumo}

Nos últimos anos, houve um grande avanço na computação reconfigurável, em particular em hardware que emprega Field-Programmable Gate Arrays. Porém, esse aumento de capacidade e desempenho aumentou a distância entre a capacidade de projeto e a disponibilidade de tecnologia para o desenvolvimento do projeto. As linguagens de programação imperativas de alto nível, como C, são mais apropriadas para o desenvolvimento de aplicativos complexos que as linguagens de descrição de hardware. Por isso, surgiram diversas ferramentas para o desenvolvimento de hardware a partir de código em C. A ferramenta ChipCflow, da qual faz parte este projeto, é uma delas. A execução dos programas por meio dessa ferramenta será completamente baseada em seu fluxo de dados, seguindo o modelo dinâmico encontrado nas arquiteturas de computadores a fluxo de dados, aproveitando ao máximo o paralelismo considerado natural desse modelo e as características do hardware parcialmente reconfigurável. Neste projeto em particular, o objetivo é a prova de conceito (proof of concept) para a criação de instâncias, em forma de operadores, de um algoritmo ChipCflow em hardware parcialmente reconfigurável, tendo como base a plataforma Virtex da Xilinx. 


\section{Abstract}

In recent years, reconfigurable computing has become increasingly more advanced, especially in hardware that uses Field-Programmable Gate Arrays. However, the increase of performance in FPGAs accumulated the gap between design capacity and technology for the development of the design. Imperative high-level programming languages such as $\mathrm{C}$ are more appropriate for the development of complex algorithms than hardware description languages (HDL). For this reason, many ANSI C-like programming tools for the development of hardware came to existence. The ChipCflow project, of which this project is part, is one of these tools. The execution of algorithms through this tool will be completely directed by data flow, according to the dynamic model found on Dataflow Architectures, taking advantage of its natural high levels of parallelism and the characteristics of the partially reconfigurable hardware. In this project, the objective is a proof of concept for the creation of instances, in the form of operators, of a ChipCflow algorithm on a partially reconfigurable hardware, taking as reference the Xilinx Virtex boards. 


\section{Sumário}

1 INTRODUÇÃO

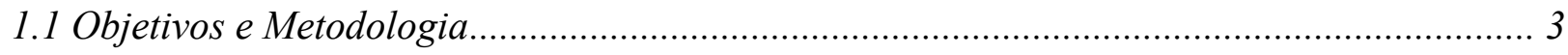

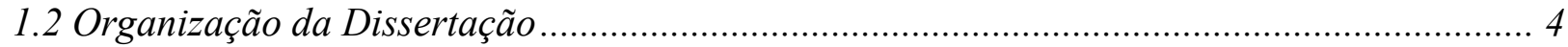

2 COMPUTAÇÃO DIRIGIDA POR FLUXO DE DADOS ....................................................................... 5

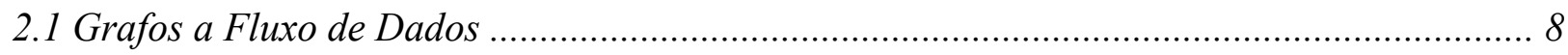

2.2 Laços Iterativos e Reentrância em Grafos .................................................................... 12

2.3 Arquiteturas Clássicas de Processadores a Fluxo de Dados................................................ 14

2.4 Arquiteturas Atuais Baseadas em Elementos de Processamento a Fluxo de Dados ............ 18

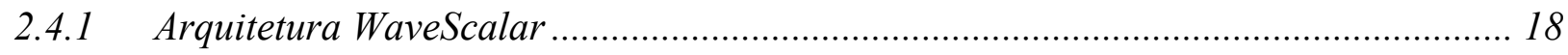

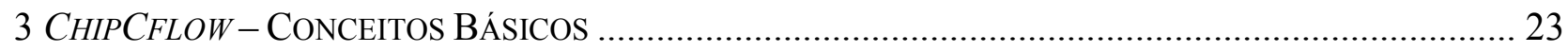

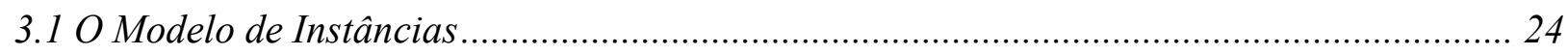

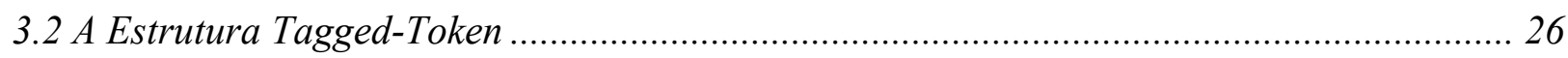

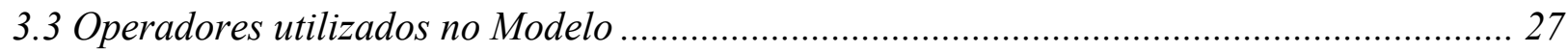

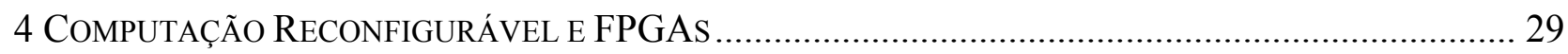

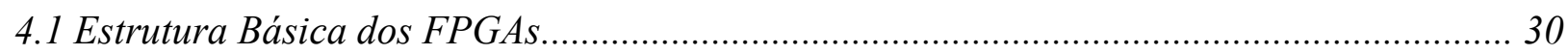

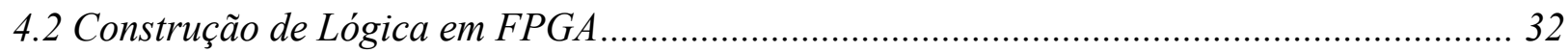

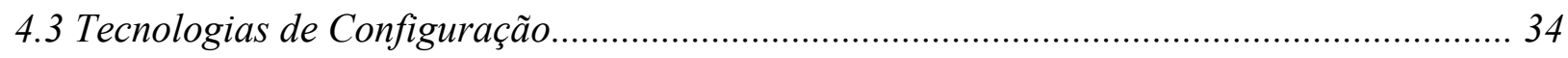

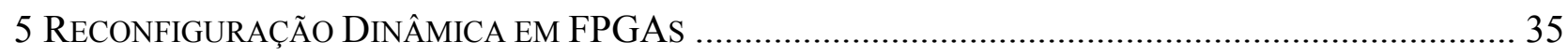

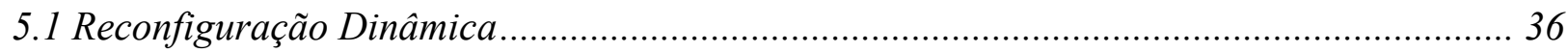

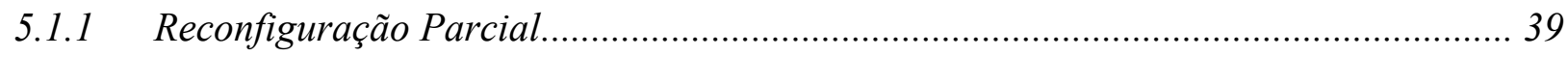

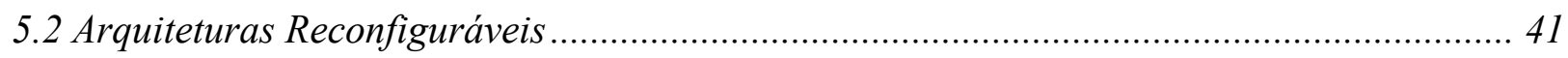

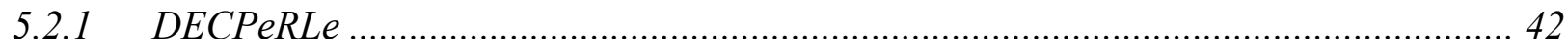

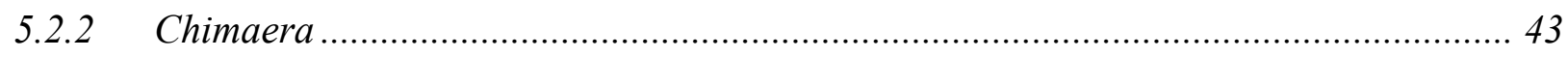

5.2.3 Garp

5.3 Reconfiguração Parcial Dinâmica nas Arquiteturas Virtex da Xilinx.................................. 46

5.3.1 Reconfiguração Parcial Baseada em Módulos ........................................................ 49

5.3.2 Reconfiguração Parcial Baseada em Diferenças....................................................... 52

6 Modelo de Reconfiguração Parcial para o ChipCflow ………………………................. 55 


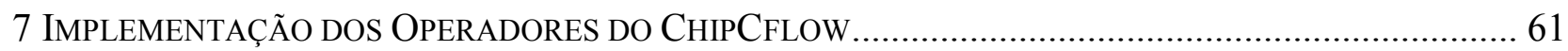

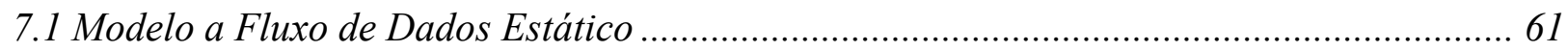

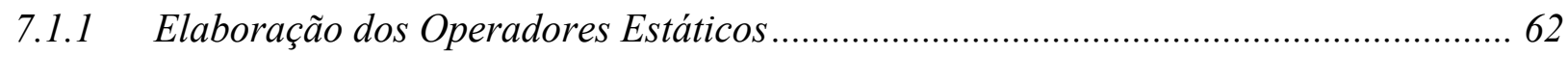

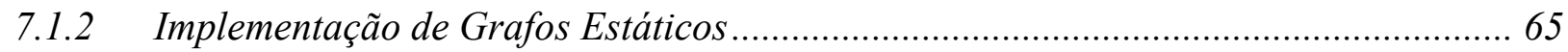

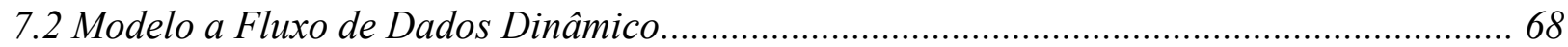

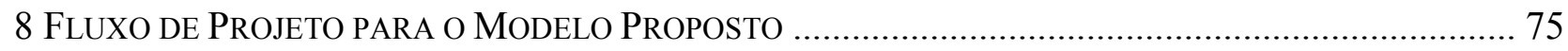

8.1 Primeiro passo: descrição do projeto em HDL e síntese .................................................... 77

8.2 Segundo passo: definição das regiões de confinamento dos elementos do projeto ............... 82

8.3 Terceiro passo: implementação do projeto estático e dos módulos reconfiguráveis............ 85

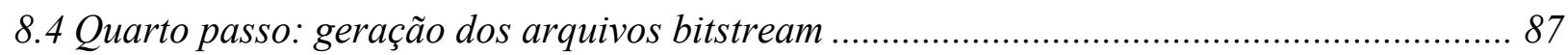

8.5 Avaliação do overhead da reconfiguração parcial............................................................. 88

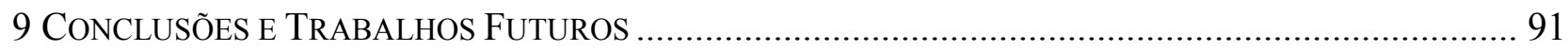

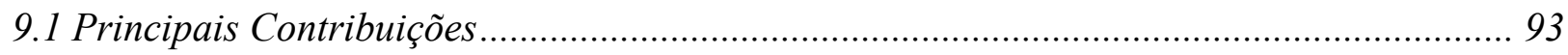

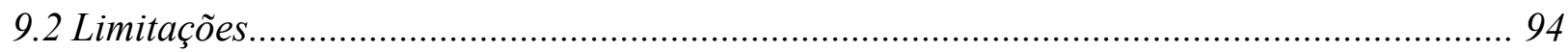

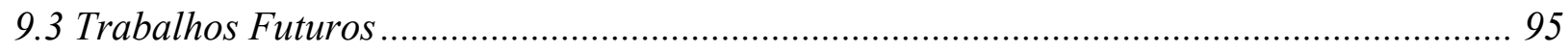

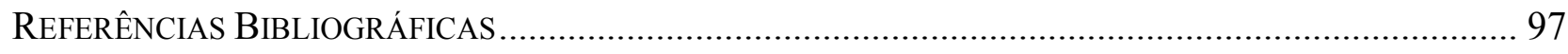

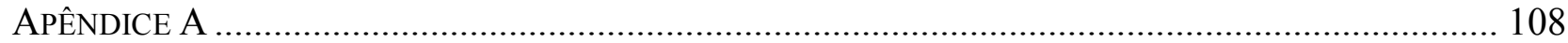

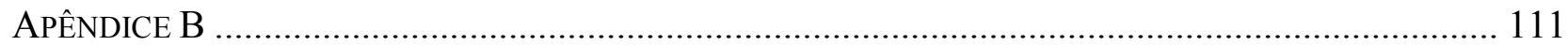

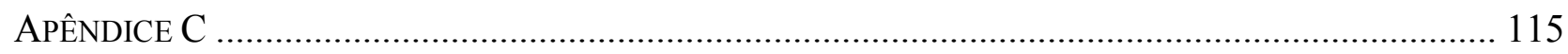




\section{Índice de Figuras e Tabelas}

Figura 2.1 - Representação em grafo Dataflow do comando de atribuição $U=f(x, y)=(x *(x+y)$ $(\mathrm{x}+\mathrm{y}) / \mathrm{y}) /(\mathrm{x} *(\mathrm{x}+\mathrm{y}) *(\mathrm{x}+\mathrm{y}))$, adaptado de [Hwang \& Briggs 1984] ..................................... 9

Figura 2.2 - Tipos de enlace dos grafos Dataflow. ............................................................ 10

Figura 2.3 - Primitivas decider (a) e branch (b) dos grafos Dataflow..................................... 10

Figura 2.4 - Primitivas value deterministic merge (a) e nondeterministic merge (b)................. 11

Figura 2.5 - Primitivas copy (a) e operator (b) ...................................................................... 11

Figura 2.6 - Grafos cíclicos problemáticos. No grafo à esquerda, haverá estado de deadlock; no grafo à direita, os operadores nunca cessarão de disparar. Adaptado de [Veen 1986]........ 12

Figura 2.7 - Grafo iterativo com reentrância utilizando o método da fechadura, adaptado de Veen

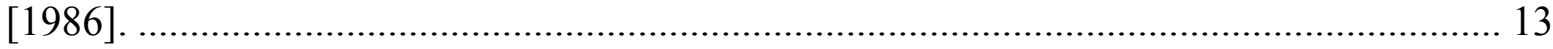

Figura 2.8 - Célula de instrução no modelo estático. Adaptado de [Dennis \& Misunas 1975].... 14

Figura 2.9 - Formato de uma instrução. Adaptado de [Dennis \& Misunas 1975]...................... 15

Figura 2.10 - Organização básica do modelo a fluxo de dados estático [Dennis 1980].............. 15

Figura 2.11 - Organização básica do modelo a fluxo de dados dinâmico [Lee \& Hurson 1993]. 16

Figura 2.12 - WaveScalar: Diagrama de blocos de um elemento de processamento [Swanson et

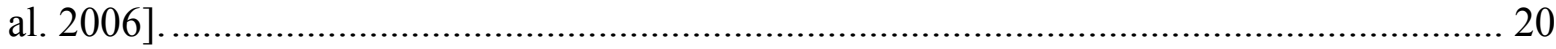

Figura 2.13 - Exemplo de mapeamento de código fonte no processador WaveScalar [Swanson et al. 2006]. 21

Figura 2.14 - Organização hierárquica da arquitetura WaveScalar: Processadores agrupados em

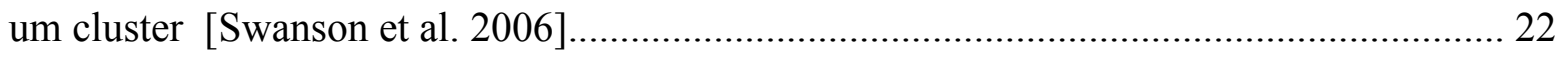

Figura 3.1 - Diagrama de fluxo da ferramenta ChipCflow.................................................. 23

Figura 3.2 - Sub-grafo F a ser incluído no grafo T [Silva 2006] ........................................... 25

Figura 3.3 - Resultado da concatenação do sub-grafo F ao grafo T [Silva 2006]...................... 25

Figura 3.4 - Processo de criação de diferentes instâncias do operador "“*”. .............................. 27

Figura 3.5 - Operadores utilizados no modelo ChipCflow [Silva \& Marques 2006]................. 28

Figura 3.6 - Em (a) está representado um grafo que representa um comando de atribuição; em (b) está representado o comando IF da linguagem C [Silva \& Marques 2006] ....................... 28

Figura 4.1 - Estrutura básica de um FPGA, adaptado de Brown \& Vranesic [2005]................. 31 
Figura 4.2 - Um bloco lógico básico de um FPGA [Compton \& Hauck 2002]. ........................ 32

Figura 4.3 - Fluxo de projeto para construção de aplicações FPGA [Nascimento et al. 2006].... 33

Figura 5.1 - Classes de configurabilidade dos FPGAs [Ribeiro 2002] .................................... 36

Figura 5.2 - Divisão de uma aplicação em duas configurações menores que podem ocupar o

FPGA em tempos diferentes [Compton \& Hauck 2002] ............................................... 37

Figura 5.3 - Os diferentes tipos de dispositivos reconfiguráveis [Skliarova \& Ferrari 2003]...... 38

Figura 5.4 - Exemplo de reconfiguração dinâmica [Ribeiro 2002] .......................................... 40

Figura 5.5 - Execução das tarefas com execução total [Ribeiro 2002] ...................................... 40

Figura 5.6 - Execução das tarefas com execução parcial [Ribeiro 2002] ................................... 40

Figura 5.7 - Arquitetura do DECPeRLe [Vuillemin et al. 1996] ............................................ 43

Figura 5.8 - Arquitetura do Chimaera [Hauck et al. 2004] .............................................. 44

Figura 5.9 - Organização básica da arquitetura Garp em diagrama de blocos [Costa 2007]........ 45

Figura 5.10 - Arquitetura dos FPGAs Virtex-II [Adaptado de Xilinx 2007a] .......................... 47

Figura 5.11 - Arquitetura dos FPGAs Virtex-4 [Xilinx 2007b] ........................................... 48

Figura 5.12 - Projeto com módulos reconfiguráveis [Xilinx 2005] ........................................ 50

Figura 5.13 - FPGA Editor mostrando o esquemático de um slice [Xilinx 2007e]................... 54

Figura 6.1 - Exemplo que ilustra o modelo de reconfiguração parcial dinâmica para o projeto. . 57

Figura 6.2 - Modelo de um módulo parcialmente reconfigurável (PRM)............................... 60

Figura 7.1 - Diagrama de máquina de estados, em notação SysML, dos operadores estáticos.... 63

Figura 7.2 - Bloco esquemático OPADD - operador estático de adição.................................. 64

Figura 7.3 - Operador OPADD: Diagrama de estados e parte arquitetural do código VHDL. .... 64

Figura 7.4 - Grafo a fluxo de dados extraído de um algoritmo que usa declarações if-else......... 66

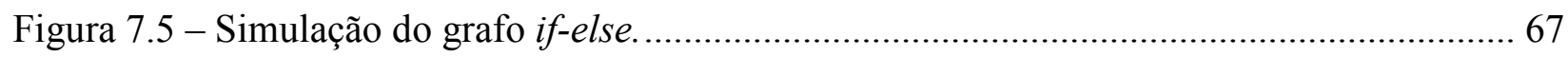

Figura 7.6 - Implementação, em blocos esquemáticos, do grafo "if-else". .............................. 67

Figura 7.7 - Diagrama de máquina de estados, em notação SysML, representando a versão

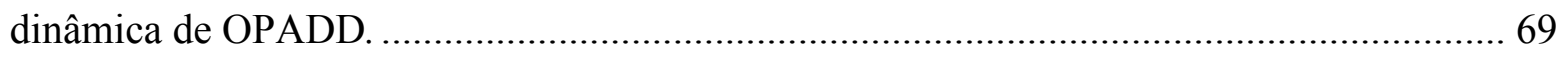

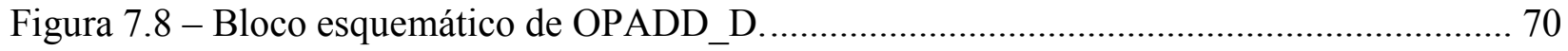

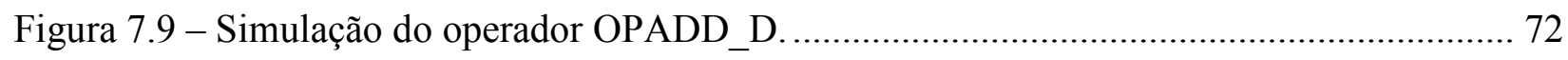

Figura 7.10 - Bloco esquemático de OPADD_R............................................................... 72

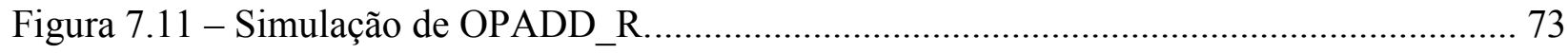

Tabela 7.1 - Utilização lógica dos módulos OPADD_D e OPADD_R ..................................... 74 
Figura 8.1 - Grafo implementado como prova de conceito inicial para o modelo proposto. ....... 76

Figura 8.2 - Passos básicos do fluxo de projeto parcialmente reconfigurável........................... 77

Figura 8.3 - Instanciação do clock, em nível de topo............................................................ 78

Figura 8.4 - Instanciação do bus macro 1 (BM1)................................................................ 79

Figura 8.5 - Declaração dos sinais de entrada e saída do arquivo de topo do projeto................. 79

Figura 8.6 - Instanciação do módulo parcialmente reconfigurável (PRM). ............................. 80

Figura 8.7 - Simulação do projeto descrito no nível de topo................................................ 81

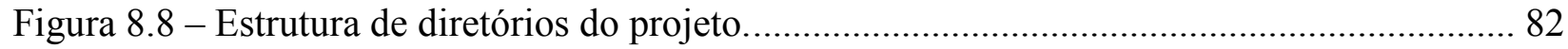

Tabela 8.1 - Sumário da utilização lógica de um dispositivo Virtex-II Pro pelo projeto plano. .. 82

Figura 8.9 - Importação de netlists durante criação de projeto no PlanAhead. ........................... 83

Figura 8.10 - Criação de um Pblock para os módulos estáticos. ............................................. 84

Figura 8.11 - Pblock no qual a PRR é confinada e seus bus macros correspondentes................ 85

Figura 8.12 - Porcentagem de uso de cada elemento lógico por OPMULT_R no PRR.............. 86

Figura 8.13 - Porcentagem de uso de cada elemento lógico por OPADD_R no PRR. ............... 86

Figura 8.14 - O painel ExploreAhead Runs, utilizado durante o processo de MAP, PAR e

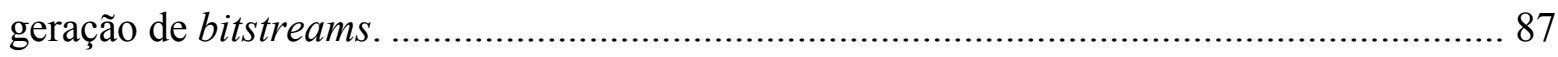

Figura 8.15 - Arquivos bitstream gerados, incluindo os parciais de cada PRM...................... 88

Tabela 8.2 - Tamanho dos bitstreams gerados. ............................................................... 88 
xviii 


\section{Lista de Siglas}

ANSI

ASIC

CAM

CDFG

CLB

CPU

DCM

DFG

DSP

EDA

EEPROM

EPROM

E/S

FPGA

GPP

GR

GRM

HDL

I/O

ICMC

ILP

IOB

ISE

JTAG

LUT

MIPS

MIT

OPB
American National Standards Institute

Application-Specific Integrated Circuit

Content-Addressable Memory

Control/Data Flow Graph

Configurable Logic Block

Central Processing Unit

Digital Clock Manager

Dataflow Graph

Digital Signal Processing

Electronic Design Automation

Electrically Erasable Programmable Read-Only Memory

Erasable Programmable Read-Only Memory

Entrada / Saída

Field-Programmable Gate Array

General Purpose Processor

Gerenciador de Reconfigurações

General Routing Matrix

Hardware Description Language

Input / Output

Instituto de Ciências Matemáticas e de Computação

Instruction-Level Parallelism

Input / Output Block

Integrated Software Environment

Joint Test Action Group

Look-up Table

Microprocessor without Interlocked Pipeline Stages

Massachusetts Institute of Technology

On-chip Peripheral Bus 


\begin{tabular}{|c|c|}
\hline OTF & On-the-fly \\
\hline PAM & Programmable Active Memories \\
\hline $\mathbf{P E}$ & Processing Element \\
\hline PRISC & PRogrammable Instruction Set Computers \\
\hline PRM & Partial Reconfiguration Module \\
\hline PROM & Programmable Read-Only Memory \\
\hline $\mathbf{P R}$ & Partially Reconfigurable \\
\hline PRR & Partially Reconfigurable Region \\
\hline PRTR & Partial Run-Time Reconfiguration \\
\hline $\mathbf{R A M}$ & Random Access Memory \\
\hline RTL & Register Transfer Level \\
\hline RTR & Run-Time Reconfiguration \\
\hline SoPC & System on Programmable Chip \\
\hline SPEC & Standard Performance Evaluation Corporation \\
\hline SysML & Systems Modeling Language \\
\hline SRAM & Static Random Access Memory \\
\hline SRL & Shift Register Look Up Table \\
\hline TBUF & Tristate Buffer \\
\hline TLP & Thread-Level Parallelism \\
\hline ULA & Unidade Lógica e Aritmética \\
\hline URL & Uniform Resource Locator \\
\hline USP & Universidade de São Paulo \\
\hline VHDL & VHSIC Hardware Description Language \\
\hline VHSIC & Very-High-Speed Integrated Circuits \\
\hline VLIW & Very Long Instruction Word \\
\hline
\end{tabular}




\section{Introdução}

Apesar de ser aceitável que a lei de Moore, que prevê a duplicação da quantidade de transistores em um chip aproximadamente a cada 18 meses, continuará sendo válida no futuro próximo [Intel 2005], limitações físicas impossibilitam que essa escalabilidade seja obtida mantendo-se as tecnologias e técnicas atuais, uma vez que o simples aumento da quantidade de transistores não necessariamente se converte em aumento de desempenho. Essencialmente, há três barreiras a serem transpostas: escalabilidade, potência, e memória [Ronen 2001] [Gelsinger 2001] [Collins 2003] [Spracklen \& Abraham 2005] [Parkhurst et al. 2006] [Borkar 2007]. A primeira barreira, de escalabilidade, se refere: (1) à crescente complexidade dos circuitos, o que aumenta o time-to-market e a possibilidade de inserção de bugs de projeto no circuito; (2) à crescente disparidade entre o desempenho dos processadores e da comunicação dentro do chip; (3) e à decrescente confiabilidade da tecnologia dos circuitos, devido ao processo de miniaturização de seus componentes. Já a segunda barreira, de potência, é resultado do aumento da quantidade de energia gasta pelo chip por unidade de área, que leva a um decréscimo da eficiência devido ao aumento da temperatura. Finalmente, a barreira de memória ilustra as limitações ocasionadas pelo compartilhamento de barramentos e da largura de banda de memória, que constituem gargalos da arquitetura de von Neumann.

A arquitetura baseada em fluxo de dados, ou dataflow, é uma alternativa radical à arquitetura tradicional de von Neumann [Dennis \& Misunas 1975] [Dennis 1980] [Veen 1986] [Arvind 2005]. Na arquitetura a fluxo de dados, que é naturalmente paralela, a execução é 
dirigida apenas pela disponibilidade do conjunto de dados, eliminando a necessidade da existência de um program counter e de memória comum centralizada. Como resultado, a execução de um programa tem um alto grau de paralelismo, se for possível paralelizar o programa. Em todas as arquiteturas a fluxo de dados, um programa é convertido em um grafo dataflow (DFG), onde as dependências de controle são convertidas em dependência de dados. Entretanto, várias limitações do modelo a fluxo de dados puro dificultaram a implementação de máquinas com utilidade prática [Gajski et al. 1982] [Lee \& Hurson 1993].

Aplicativos científicos e de engenharia necessitam de capacidade de processamento cada vez maior, requerendo, muitas vezes, a execução diretamente em hardware. FPGA (Fieldprogrammable Gate Array) é um tipo de hardware que permite reconfiguração, possibilitando a alteração de suas funcionalidades conforme a necessidade de seus usuários, combinando, assim, o desempenho do hardware dedicado com a flexibilidade alcançada pelo software. Embora FPGAs sejam uma boa alternativa para a implementação de algoritmos em hardware, linguagens de programação de alto-nível são mais apropriadas que as linguagens de descrição de hardware (HDL) atuais para descrever aplicativos complexos. A utilização de HDLs requer etapas de desenvolvimento demoradas e complexas, se comparadas com linguagens imperativas de altonível como C ou Java, além de exigirem conhecimentos específicos de hardware. Por isso, é interessante estudar ferramentas automáticas para geração de hardware a partir de linguagens de alto-nível [Cardoso \& Neto 2003] [Pellerin \& Thibault 2005] [Lopes et al. 2006] [Lopes 2007]. Outra vantagem obtida com isso é a possibilidade de reuso de algoritmos já implementados em linguagens com grande utilização.

Alguns FPGAs permitem a reconfiguração parcial em tempo de execução, possibilitando a criação de ferramentas de compilação que geram hardware, dinamicamente, a partir de algoritmos implementados em uma linguagem de alto nível, que podem ser convertidos para grafos DFG [Silva \& Marques 2006]. Muitas arquiteturas em hardware reconfigurável foram propostas nos últimos anos, tendo como finalidade a aceleração de algoritmos por meio da execução em FPGA das partes custosas computacionalmente. Como exemplo, pode-se citar a arquitetura Garp [Callahan et al. 2000], a Chimaera [Hauck et al. 2004], entre muitas outras. 
A ferramenta ChipCflow, em desenvolvimento desde o início de 2006, é um projeto de arquitetura a fluxo de dados dinâmica em hardware reconfigurável, tendo como objetivo a aceleração de programas escritos na linguagem $\mathrm{C}$, em particular as partes que demandam maior capacidade de processamento, por meio da execução direta em hardware, tendo como benefício o paralelismo natural do modelo a fluxo de dados e a versatilidade e desempenho do hardware parcialmente reconfigurável [Silva 2006] [Silva \& Marques 2006] [Lopes et al. 2006] [Astolfi \& Silva 2007] [Souza 2008] [Costa 2009] [Silva et al. 2009].

\subsection{Objetivos e Metodologia}

O objetivo geral deste trabalho é a implementação do processo de reconfiguração parcial dinâmica em FPGAs aplicado ao modelo de instâncias da ferramenta ChipCflow. Será oferecido um modelo que contempla a criação e execução de instâncias, em forma de operadores, de programas a fluxo de dados dinâmico específico do ChipCflow, além de uma prova de conceito (proof of concept) para esse modelo, utilizando os recursos oferecidos pela reconfiguração parcial dinâmica, tendo como resultado os bitstreams necessários à programação parcial do FPGA.

Como objetivos específicos para o desenvolvimento do projeto, inicialmente, baseando-se no estudo da reconfiguração parcial, foi proposto um modelo de reconfiguração para o ChipCflow. Foram criados também os operadores para o modelo a fluxo de dados estático, sendo implementados para fins de teste, a partir desses operadores, vários grafos estáticos, além de módulos auxiliares, utilizados para que a execução dos grafos ocorresse da maneira correta. Em seguida, os operadores estáticos foram adaptados para o modelo dinâmico já proposto, e foram desenvolvidos módulos auxiliares específicos desse modelo. Finalmente foi desenvolvido, como prova de conceito, um algoritmo na forma de grafo a fluxo de dados tendo como base o modelo proposto.

O método utilizado para o desenvolvimento do projeto requereu o estudo de ferramentas e técnicas disponíveis para a plataforma Virtex da Xilinx, que suporta a reconfiguração parcial dinâmica. Utilizou-se uma linguagem de descrição de hardware (hardware description language - HDL) para o desenvolvimento dos operadores na ferramenta Xilinx ISE, cujos testes foram 
feitos utilizando-se o software ISE Simulator, já incorporado ao ISE. Utilizou-se o software Xilinx PlanAhead durante o fluxo de projeto parcialmente reconfigurável devido às características particulares desse fluxo.

\subsection{Organização da Dissertação}

Até o quinto, nos Capítulos desta dissertação são descritas as informações necessárias para o entendimento do projeto que foi desenvolvido. No Capítulo 1 é descrito, de maneira breve, informações introdutórias, como a contextualização e motivação do projeto. No Capítulo 2 é descrita a computação baseada em fluxo de dados, sendo apresentadas arquiteturas pioneiras e modernas. A seguir, no Capítulo 3 são apresentados os conceitos básicos da ferramenta ChipCflow, da qual este trabalho faz parte. O Capítulo 4 traz informações introdutórias a respeito de computação reconfigurável e FPGAs. Finalmente, o Capítulo 5 trata especificamente da reconfiguração dinâmica em FPGAs, dando ênfase à plataforma Virtex, da Xilinx, e às maneiras pelas quais a reconfiguração parcial dinâmica é feita nesses dispositivos.

No Capítulo 6 é descrito um modelo de reconfiguração parcial dinâmica para a criação de instâncias de programas compatível com o modelo a fluxo de dados dinâmico característico do ChipCflow. Os operadores do modelo proposto foram implementados e testados, estando descritos no Capítulo 7, e será apresentado no Capítulo 8, como prova de conceito, um grafo contemplando o modelo proposto, tendo como resultado os bitstreams parciais necessários à programação do FPGA. Por fim, no Capítulo 9 serão apresentados as conclusões e trabalhos futuros. 


\section{2}

\section{Computação dirigida por Fluxo de Dados}

Em 1965, Gordon E. Moore observou empiricamente que o número de dispositivos em um chip dobrava a cada doze meses. Além disso, previu que essa tendência continuaria nos anos 70, havendo um decréscimo nesse aumento, devido a limites de fabricação, a partir da década de 80, quando o número de dispositivos dobraria aproximadamente entre 18 a 24 meses [Moore 1965] [Moore 1975]. Essa observação, conhecida como "Lei de Moore”, descreve uma tendência importante na história dos microprocessadores e da capacidade dos dispositivos eletrônicos em geral, como memórias RAM e discos rígidos, cujo aumento também possui escala exponencial.

Com o aumento do número de transistores em um chip, a densidade de potência é a maior barreira a ser transposta pela indústria de processadores [Ronen 2001] [Parkhurst et al. 2006] [Borkar 2007]. A densidade de potência é definida como a potência dissipada pelo chip por uma unidade de área, em geral Watts $/ \mathrm{cm}^{2}$. Quando uma nova geração de microprocessadores é introduzida, ela consome duas vezes mais energia que a geração anterior. A extrapolação da dissipação de potência sugere, por exemplo, que os microprocessadores poderiam atingir a temperatura de um reator nuclear em 2010 [Collins 2003]. Em um chip, é essencial controlar a temperatura para que haja maior desempenho e menor desperdício de energia. 
Evidentemente, novas técnicas de fabricação e materiais estão sendo pesquisadas, e transistores com nodos menores estão se tornando economicamente viáveis [Boyd et al. 2007]. Porém, como é cada vez mais difícil extrair mais desempenho dessa maneira, a indústria, para suprir a demanda por mais capacidade de processamento, recorreu aos processadores multi-core [Parkhurst et al. 2006] [Flynn \& Hung 2005], capazes de oferecer paralelismo em nível de thread (Thread-Level Parallelism - TLP). O paralelismo é uma das melhores maneiras de lidar com a densidade de potência porque permite maior capacidade de processamento com menor demanda de voltagem e freqüência. Assim, o foco de atenção passa a ser o número de núcleos de processamento e a operação multitarefa, e não a freqüência de clock.

Entretanto, o poder de processamento de um chip não depende apenas do desempenho do processador, havendo limitações causadas pelo compartilhamento de barramentos e da largura de banda de memória, que são gargalos típicos da arquitetura de von Neumann [Gelsinger 2001] [Spracklen \& Abraham 2005] [Borkar 2007], uma vez que o aumento da freqüência dos barramentos externos não acompanhou o aumento da freqüência dos processadores. Por isso, a latência de acesso à memória aumentou com o tempo, prejudicando cada vez mais o desempenho do sistema.

Alguns conceitos de processamento orientado a fluxo de dados (data flow) já são aplicados nos processadores atuais denominados superescalares, pois o que importa durante o processamento não é a ordem das instruções, mas as dependências de dados entre elas. Assim, várias técnicas para obtenção de paralelismo em nível de instrução (instruction-level parallelism ILP) foram desenvolvidas, como pipeline, execução fora-de-seqüência, e execução especulativa. Durante a última década, as técnicas de ILP sozinhas forneceram um aumento de desempenho de aproximadamente $600 \%$ [Gelsinger 2001], porém com o aumento tanto da complexidade do chip quanto da potência consumida. Contudo, como demonstrou Ronen [2001], o aumento do número de estágios de pipeline indefinidamente não é uma boa solução, por causa do crescente aumento da potência consumida, em contraste com o baixo speedup. Como foi visto, densidade de potência é um conceito-chave no que se refere ao projeto dos processadores atuais.

Um objetivo dos projetistas de microprocessadores é obter o máximo de paralelismo em nível de instrução a ponto de tentar quebrar, de maneira eficiente, a barreira da dependência de 
dados, por meio de execuções especulativas [Pollack 1999]. Entretanto, como mostra Spracklen \& Abraham [2005], há um aumento exponencial da comunicação fora do chip ao se utilizar técnicas especulativas avançadas para previsão de desvio e de valor. Além disso, a execução de instruções com dados erroneamente especulados diminui a eficiência energética do processador.

Devido a esses problemas e desafios, é consenso que a indústria de microprocessadores não mais poderia operar da maneira tradicional, e pesquisadores, tanto o meio acadêmico quanto industrial, estão procurando por novas alternativas no âmbito arquitetural, de sistema, de algoritmos, e de materiais. A introdução dos processadores multi-core é uma delas [Parkhurst et al. 2006] [O'Reilly 2007] [Wolfe 2008], que no futuro atingirão o ponto de ter muitos núcleos, sendo assim chamados many-cores [Borkar 2007].

Embora o conceito de computação dirigida pelo fluxo de dados, ou Dataflow, seja tão antigo quanto a computação eletrônica [Veen 1986], somente nas décadas de 1970 e 1980 as arquiteturas de processadores a fluxo de dados tiveram grande destaque no meio acadêmico. Jack Dennis, do MIT, foi pioneiro no desenvolvimento de esquemas para representação de programas Dataflow, que mais tarde foram chamados grafos Dataflow (DFG). Dennis e seu grupo de pesquisa também foram pioneiros no desenvolvimento das máquinas a fluxo de dados estáticas [Dennis \& Misunas 1975] [Dennis 1980], enquanto a Arquitetura Dataflow por Tagged-Token do MIT [Gurd et al. 1985] e a Máquina Dataflow de Manchester [Arvind \& Nikhil 1990] foram os primeiros trabalhos no que se refere ao modelo dinâmico, cuja diferença em relação ao estático será explicada na Seção 2.2. Apesar de nenhum computador com arquitetura Dataflow ter tido viabilidade comercial, esse modelo computacional influenciou muitas áreas, como linguagens de programação, compilação paralela, processamento de sinais digitais, projeto de processadores, arquiteturas com multitarefa, e na alocação de objetos na linguagem Java [Laskowski et al. 2004], entre outras aplicações. Nos processadores superescalares modernos, a execução fora-deseqüência, que é uma forma restrita de Dataflow, tornou-se dominante a partir da década de 1990.

A arquitetura a fluxo de dados explora, de modo simples e natural, paralelismo de granularidade fina, uma vez que execução de cada instrução é dirigida somente pela disponibilidade de seus valores de entrada. Por este motivo, máquinas a fluxo de dados têm execução assíncrona, que é uma característica desejável para o processamento paralelo. Nesta 
arquitetura, alguns gargalos característicos da arquitetura de von Neumann são eliminados, uma vez que não há necessidade de existir um program counter ou armazenamento de dados compartilhado. Ao explorar o paralelismo em nível de instrução, o modelo a fluxo de dados também é capaz de explorar o paralelismo em nível de linhas de execução, conhecidas normalmente como processos, mediante conjuntos de instruções separadas que estão prontas para executar quando seus respectivos dados de entrada estão disponíveis. Assim, computadores Dataflow têm a capacidade de explorar todo o paralelismo disponível em um programa [Hwang 1993], se não considerarmos execuções especulativas.

\subsection{Grafos a Fluxo de Dados}

No modelo Dataflow, um programa é representado por um grafo dirigido, onde os vértices representam as instruções (operadores) e os arcos representam as dependências de dados entre os vértices. Os dados são transportados nos arcos por pacotes chamados tokens. Conceitos comuns em linguagens de programação, como variáveis e atualização de memória, não existem no modelo Dataflow. Ao invés disso, objetos, que podem ser tanto estruturas de dados quanto valores escalares, são consumidos por um operador que, por sua vez, produzirá um objeto resultante que será passado aos operadores subseqüentes. Nas arquiteturas Dataflow, um mesmo objeto pode ser enviado, mediante cópia, para diferentes operadores ao mesmo tempo.

Quando um operador é executado, diz-se que ocorreu um “disparo” (firing). Isso só ocorre se o operador está habilitado (enabled), o que é determinado por uma regra de habilitação. Normalmente, é definida uma regra de habilitação estrita, o que significa que um operador é habilitado somente quando todas as portas de entrada contêm um token.

O seguinte código em $\mathrm{C}$ ilustra a execução de comando de atribuição em grafos Dataflow:

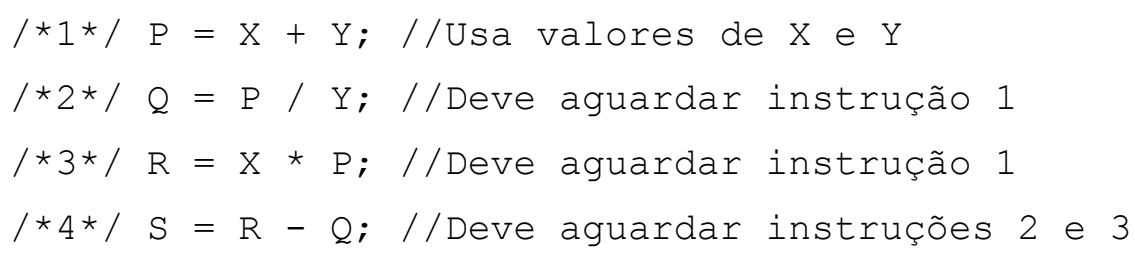




$$
\begin{aligned}
& / * 5 * / \mathrm{T}=\mathrm{R} * \mathrm{P} ; / / \text { Deve aguardar instruções } 1 \text { e } 3 \\
& / * 6 * / \mathrm{U}=\mathrm{S} / \mathrm{T} ; / / \text { Deve aguardar instruções } 4 \text { e } 5
\end{aligned}
$$

Em um computador escalar com processamento serial, seria possível as seguintes seqüências de execução, todas com seis passos:

$$
\begin{aligned}
& (1,2,3,4,5,6) \quad(1,3,2,5,4,6) \\
& (1,3,5,2,4,6) \quad(1,2,3,5,4,6) \quad(1,3,2,4,5,6)
\end{aligned}
$$

Como já mencionado, no modelo Dataflow a linguagem de máquina é representada por grafos Dataflow (DFG). A Figura 2.1 mostra o DFG para esse comando de atribuição.

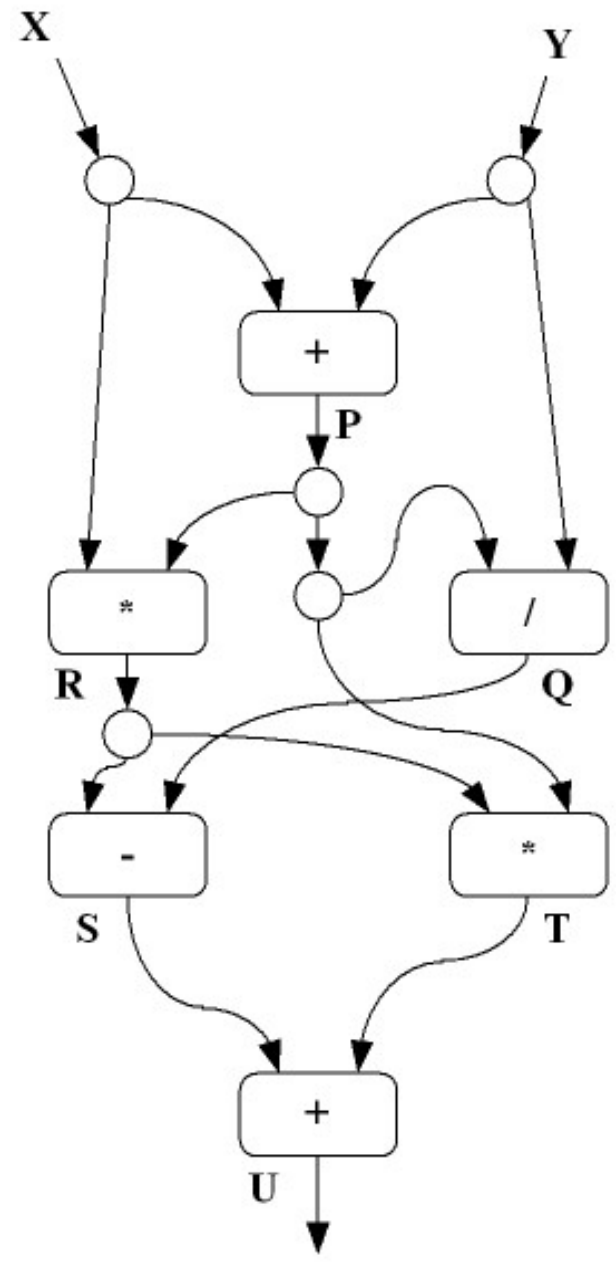

Figura 2.1 - Representação em grafo Dataflow do comando de atribuição $U=f(x, y)=(x *(x+y)-(x+y) / y) /(x *(x+y) *(x+y))$, adaptado de [Hwang \& Briggs 1984]. 
Pode-se notar que, no grafo, os nós responsáveis pelas operações são representados por retângulos com bordas arredondadas, e os responsáveis pela cópia do dado por círculos. A representação dos operadores Dataflow é, em geral, arbitrária, uma vez que encontramos diversas delas na literatura. No projeto ChipCflow, assim como neste trabalho, as representações definidas por Silva [1992] serão utilizadas. Como mostra a Figura 2.2, há dois tipos de enlace sobre um grafo Dataflow, um representado por linhas contínuas, que transporta dados ou estruturas de dados (data link), e outro que transporta valores booleanos, que são utilizados com finalidade de controle (logical link), representado por linhas tracejadas.

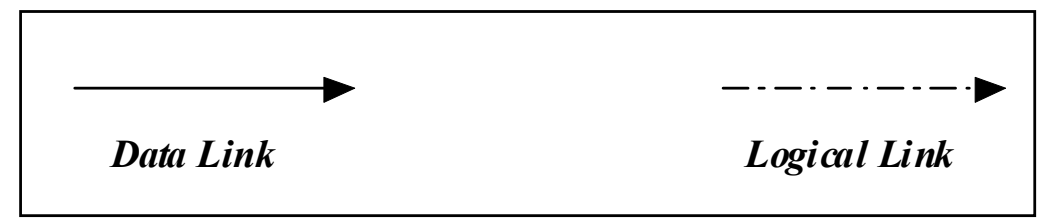

Figura 2.2 - Tipos de enlace dos grafos Dataflow.

Os operadores de ramificação (branch), junção (merge) e decisão (decider), são usados para representar computações iterativas ou condicionais no grafo. $\mathrm{O}$ nó decider, como mostra a Figura 2.3a, gera um pacote (ou token) de controle, verdadeiro ou falso, dependendo de seus valores de entrada e da função de teste T. Em um nó de ramificação (branch), ilustrado pela Figura 2.3b, uma cópia do token absorvido pela porta de entrada é colocada no arco de saída verdadeiro $(\mathrm{T})$ ou falso $(\mathrm{F})$, dependendo do valor booleano do token de controle.

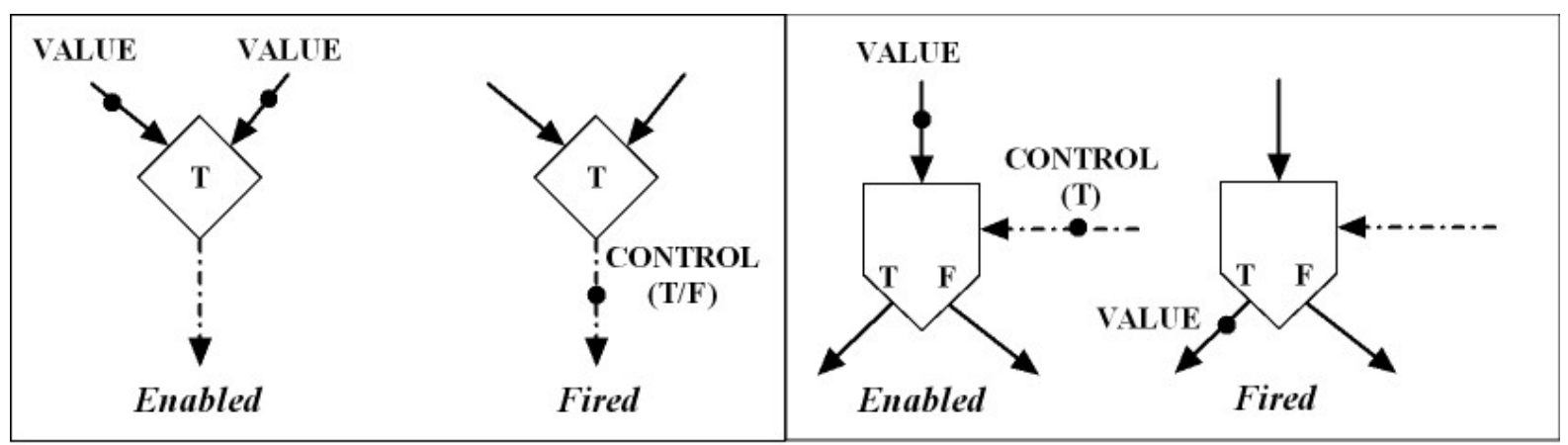

(a) Decider (Decisor)

(b) Branch (Ramificação)

Figura 2.3 - Primitivas decider (a) e branch (b) dos grafos Dataflow. 
Nos DFGs, pacotes de dados podem ser dirigidos por meio dos nós de junção (merge). Em uma junção determinística por valor (value deterministic merge), um token de controle, que pode assumir valor verdadeiro ou falso, determina de qual porta de entrada um token é absorvido (ambos devem estar presentes). Uma cópia do token absorvido é enviado para o arco de saída. O token não escolhido é descartado. Na junção não-determinística (nondeterministic merge), não existe uma regra de habilitação estrita, isto é, o nó é habilitado tão logo uma de suas portas de entrada contenha um token. Quando ele dispara, uma cópia de seu token de entrada é enviada para seu nó subseqüente. Os operadores de junção são mostrados na Figura 2.4.

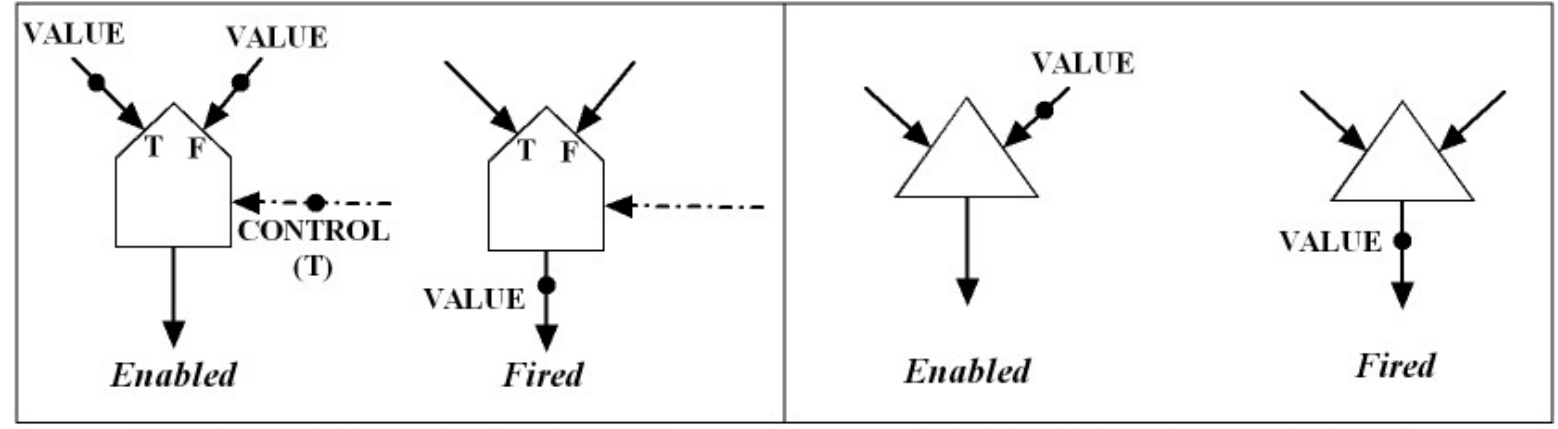

(a) Value Deterministic Merge

(b) Nondeterministic Merge

Figura 2.4 - Primitivas value deterministic merge (a) e nondeterministic merge (b).

Como já visto, um token de dado é produzido por um operador (operator) como resultado de alguma operação aritmética ou lógica, $f$. Finalmente, copiador (copy) é um operador que duplica tokens de entrada. A Figura 2.5 mostra estes operadores.

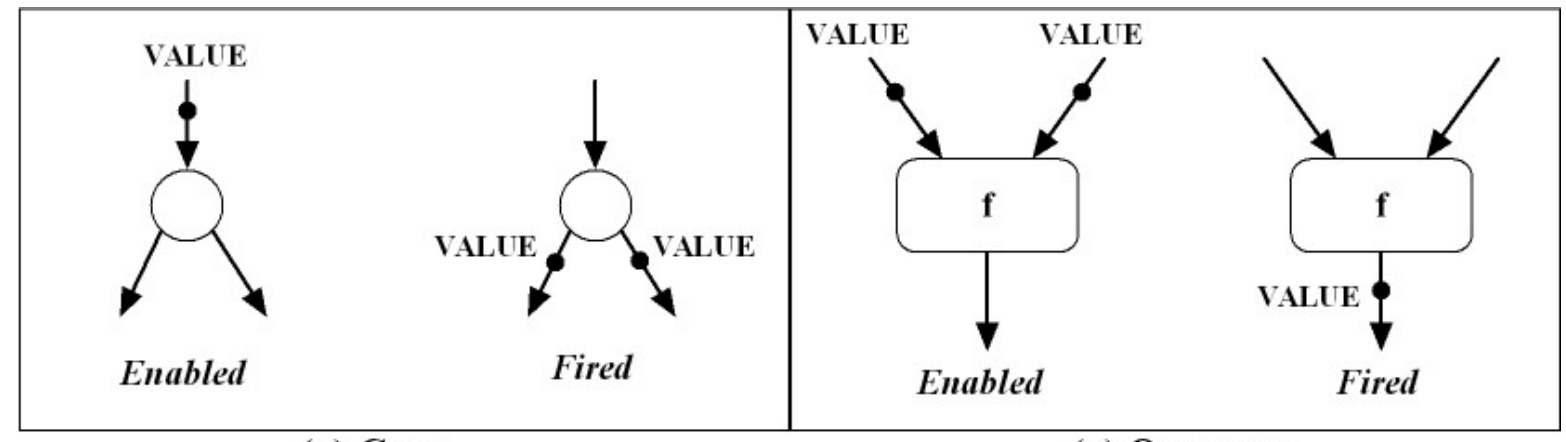

(a) Copy

(a) Operator

Figura 2.5 - Primitivas copy (a) e operator (b). 


\subsection{Laços Iterativos e Reentrância em Grafos}

Em grafos a fluxo de dados, problemas podem ocorrer quando há ciclos [Veen 1986]. A Figura 2.6 ilustra exemplos desses problemas. No grafo à esquerda, ocorrerá estado de deadlock, a não ser que se forneça algum valor inicial à porta da direita do operador de adição. Os operadores do grafo à direita nunca cessarão de disparar, uma vez disparado o merge nãodeterminístico. Embora não sejam realísticos, esses grafos ilustram os problemas que podem surgir em qualquer grafo cíclico quando as devidas precauções não são tomadas.
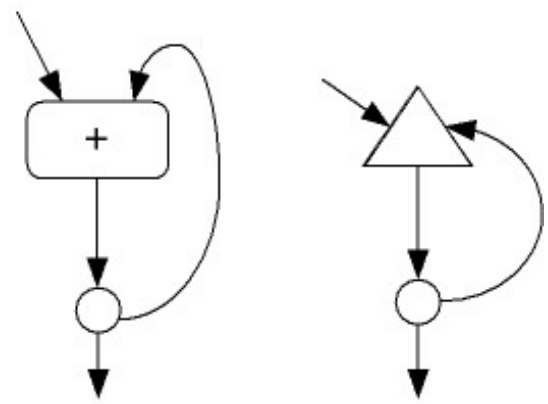

Figura 2.6 - Grafos cíclicos problemáticos. No grafo à esquerda, haverá estado de deadlock; no grafo à direita, os operadores nunca cessarão de disparar. Adaptado de [Veen 1986].

A Figura 2.7 [Veen 1986] mostra uma maneira correta de se implementar uma construção iterativa, com laços. Nesse exemplo, os operadores lidam simultaneamente com um conjunto de dois dados, x e y, apesar de terem funcionamento igual aos descritos anteriormente. Este grafo, que implementa while $\mathrm{f}(\mathrm{x})$ do begin $(\mathrm{x}, \mathrm{y}):=\mathrm{g}(\mathrm{x}, \mathrm{y})$ end, usa o método chamado da fechadura (lock method) para proteger os sub-grafos reentrantes $f$ e $g$. Neste grafo, utiliza-se merge e branch compostos. Isso garante que, graças à sua regra de habilitação estrita, cada um desses nós compostos não dispare antes que o sub-grafo $g$ tenha liberado ambos seus tokens de saída.

O sub-grafo $g$ é um exemplo de grafo reentrante, pois seus nós podem disparar repetidamente. Se assumirmos que o sub-grafo $g$ é tal que nenhum token corre o risco de não ser utilizado em um disparo quando todos seus tokens de saída são produzidos, então os tokens que serão utilizados pela próxima iteração podem ser enviados com segurança para o mesmo subgrafo. Nesse caso, o método da fechadura não seria necessário. Esse método não é muito atraente 
em máquinas paralelas, uma vez que o nó branch atua como um obstáculo que evita a inicialização de uma nova iteração antes que a anterior é concluída.

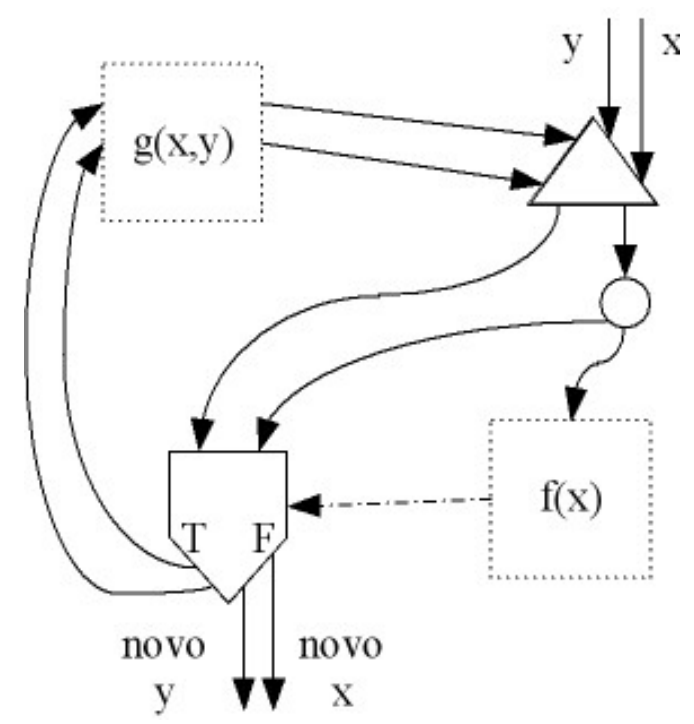

Figura 2.7 - Grafo iterativo com reentrância utilizando o método da fechadura, adaptado de Veen [1986].

Um maior nível de concorrência é obtido quando cada iteração é executada em uma instância separada, ou cópia, do sub-grafo com reentrância. Esse método com cópia de código requer uma máquina capaz de criar novas instâncias do sub-grafo e direcionar cada token para sua instância apropriada. Uma maneira eficiente de se implementar essa cópia de código é compartilhar as descrições de nó entre as diferentes instâncias do grafo sem confundir os tokens que pertencem a instâncias separadas. Isso é feito colocando-se, em cada token, uma etiqueta (tag) que identifica a que instância pertence o nó ao qual ele é direcionado. Os nós desta arquitetura, chamada tagged-token, têm uma regra de habilitação que especifica que o nó está habilitado se cada arco de entrada contém um token com tags idênticas.

A maneira como se lida com reentrância é um conceito-chave em arquiteturas Dataflow. As máquinas que lidam com a reentrância pelo método da fechadura ou outro equivalente são chamadas estáticas; as que utilizam cópia de código ou tagged-tokens são chamadas dinâmicas [Veen 1986]. Enquanto as máquinas estáticas são muito mais simples que as dinâmicas, em muitos algoritmos o nível efetivo de concorrência é menor no modelo estático. 


\subsection{Arquiteturas Clássicas de Processadores a Fluxo de Dados}

Apesar de várias arquiteturas Dataflow terem sido propostas nas últimas três décadas [Sirini 1986] [Papadopoulos \& Culler 1990] [Kavi et al. 2001] [Arul et al. 2005], pode-se destacar como os modelos clássicos, que serviram como base para os subseqüentes, a máquina estática de Jack Dennis [Dennis \& Misunas 1975] [Dennis 1980], e as máquinas dinâmicas de Manchester [Gurd et al. 1985] e do MIT [Arvind \& Nikhil 1990].

Nesta Seção será apresentada uma máquina a fluxo de dados estática que foi proposta por Dennis e seu grupo de pesquisa no MIT. No modelo, o programa que será executado é armazenado na memória do processador, que é organizada em células de instrução, sendo que cada uma delas representa um nó em um grafo Dataflow. Como mostra a Figura 2.8, cada célula de instrução é composta por três registradores. O primeiro deles armazena uma instrução, ilustrada pela Figura 2.9, que especifica a operação a ser desempenhada e os endereços dos registradores para os quais os resultados da operação são direcionados. Os outros dois registradores armazenam os dados necessários para a execução da instrução. Nesses registradores há bits de presença que indicam se o dado já está disponível.

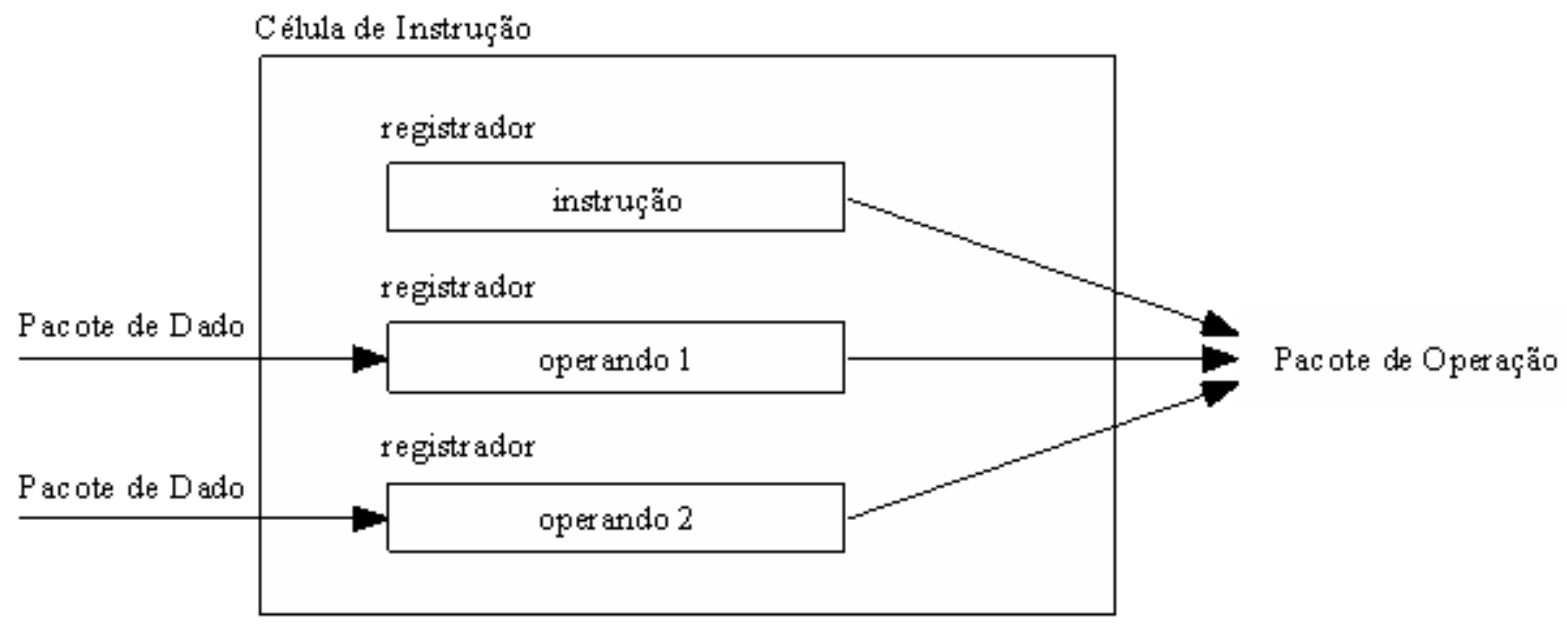

Figura 2.8 - Célula de instrução no modelo estático. Adaptado de [Dennis \& Misunas 1975]. 


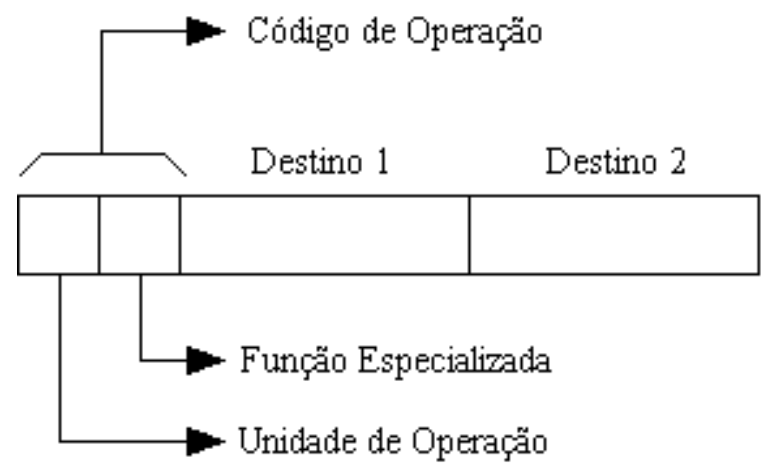

Figura 2.9 - Formato de uma instrução. Adaptado de [Dennis \& Misunas 1975].

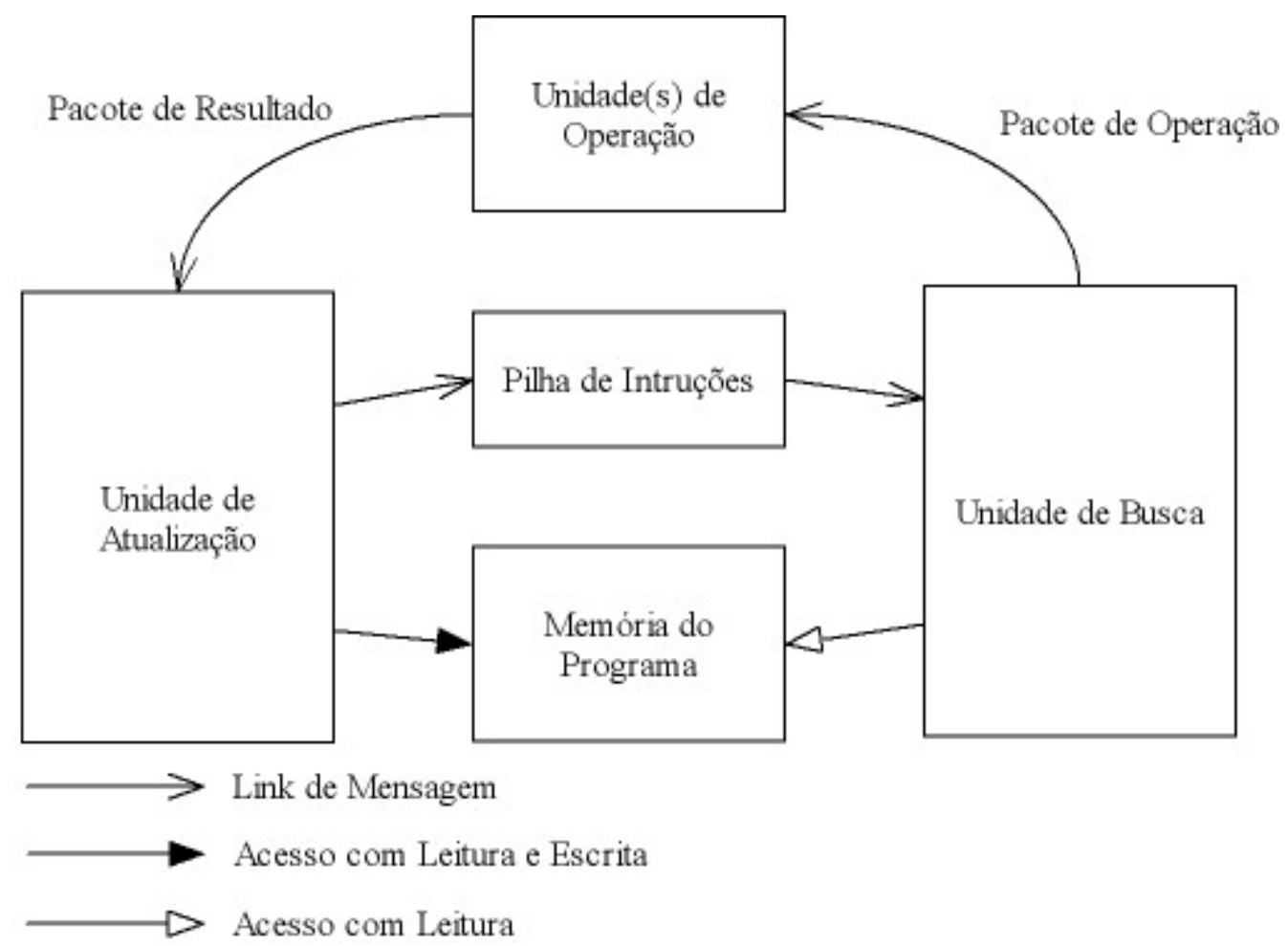

Figura 2.10 - Organização básica do modelo a fluxo de dados estático [Dennis 1980].

A organização do modelo estático, que ilustra o mecanismo básico de execução de instruções, é mostrada pela Figura 2.10. A unidade de atualização é responsável por detectar se uma instrução está habilitada para ser executada. Quando essa condição é verificada, a unidade de atualização envia o endereço da instrução habilitada para a unidade de busca, que por sua vez 
busca e envia um pacote de operação completo para a unidade de processamento, limpando no processo os bits que indicam presença de dados. A unidade de operação processa os dados, formando pacotes com resultados, e os envia à unidade de atualização, que, por fim, armazena cada resultado nos campos apropriados dos operandos, verificando os bits de presença para determinar se alguma operação foi habilitada.

Além do estático, há o modelo a fluxo de dados dinâmico, que foi proposto por Gurd, da Universidade de Manchester [Gurd et al. 1985], e por Arvind, do MIT [Arvind \& Nikhil 1990]. Nessas máquinas, vários dados podem estar em um arco ao mesmo tempo, mediante a utilização de tagged-tokens. Nessa técnica, cada token possui uma identificação, que é utilizada para deteç̧ão de tokens parceiros. Essa detecção é feita por uma unidade de matching, composta por uma memória contendo um conjunto de tokens que aguardam seus parceiros. A organização básica do modelo a fluxo de dados dinâmico é mostrada pela Figura 2.11.

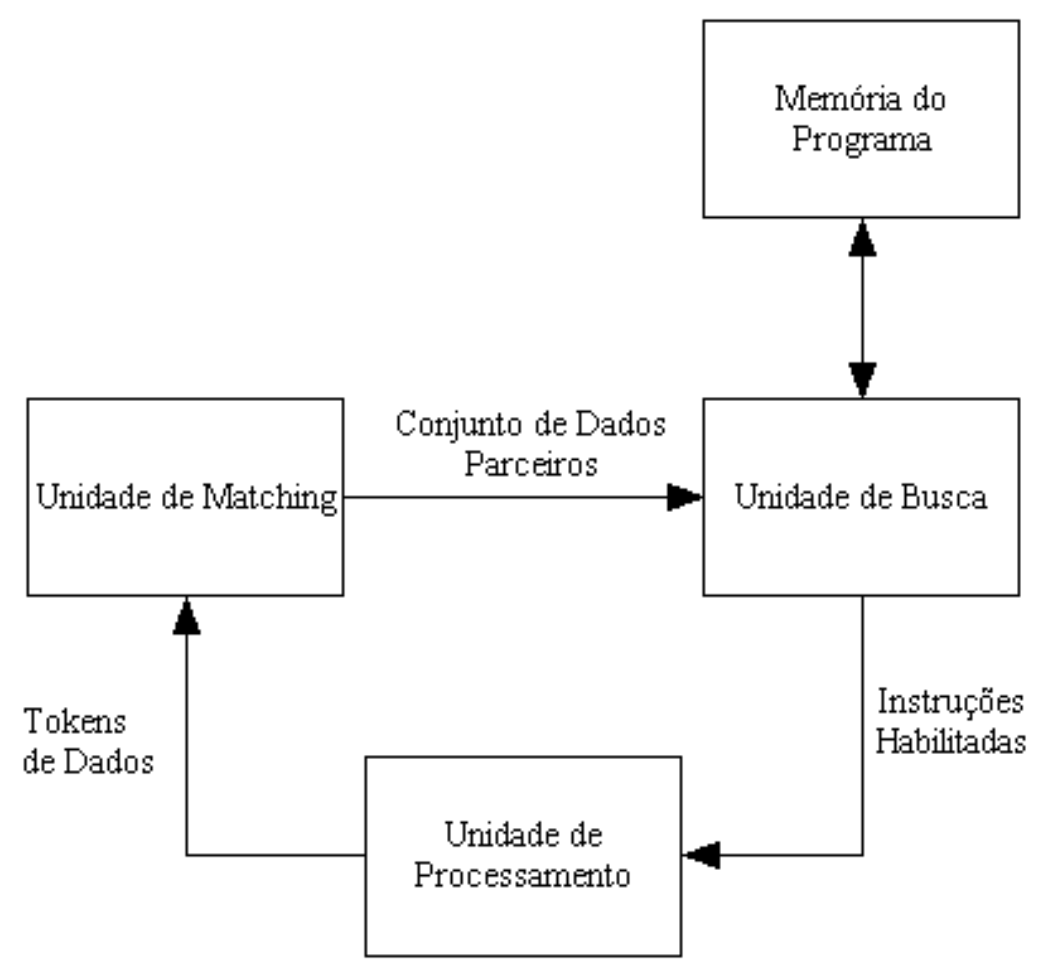

Figura 2.11 - Organização básica do modelo a fluxo de dados dinâmico [Lee \& Hurson 1993]. 
Como já explicado, no modelo dinâmico tokens são recebidos pela unidade de matching, que agrupa tokens com identificações idênticas. Se há detecção de tokens parceiros, os tokens são enviados à unidade de busca. Caso contrário, o token é armazenado na unidade de matching e espera por seu parceiro. Na unidade de busca, as tags dos tokens são utilizadas para identificar a instrução a ser buscada da memória. Finalmente, a unidade de processamento executa a instrução habilitada e produz os tokens resultantes, que serão enviados à unidade de matching.

A maior vantagem do modelo dinâmico sobre o estático é que o primeiro, ao permitir que múltiplos tokens existam em um arco, possibilita um maior nível de concorrência e, portanto, maior desempenho. Apesar de prover um maior nível de paralelismo, o overhead causado pelo processo de identificação de tokens parceiros sempre foi um problema grave [Lee \& Hurson 1993]. Para que o tempo de combinação dos tokens fosse reduzido, convencionou-se o uso de memórias associativas ou pseudo-associativas nas máquinas a fluxo de dados dinâmico [Gurd et al. 1985].

Infelizmente, várias limitações do modelo Dataflow puro impossibilitaram a criação de máquinas com utilidade prática [Gajski et al. 1982] [Lee \& Hurson 1993]. Entre as limitações mais severas, pode-se citar:

- Uso de linguagens de programação próprias, que não permitiam efeitos colaterais, entre outras características úteis das linguagens de programação imperativas [Veen 1980].

- Dificuldade de utilização de hierarquias de memórias e registradores [Thoreson \& Long 1987].

- Desperdício de capacidade de processamento, devido ao overhead ocasionado pela granularidade fina, ou causado pela subutilização dos elementos funcionais do processador [Veen 1986].

- Desperdício de espaço de armazenamento, devido ao espaço ocupado pelas tags e por endereços de destino. Devido à existência dessas estruturas, dois terços de uma célula de memória é considerado overhead [Veen 1986]. 


\subsection{Arquiteturas Atuais Baseadas em Elementos de Processamento a Fluxo de Dados}

Devido aos já descritos problemas de fabricação e projeto encontrados atualmente pela indústria de processadores, muitos projetistas estão abandonando o conceito de processadores monolíticos, complexos e de alto desempenho em favor de elementos de processamento (PE), mais simples, replicados dentro do chip. Estas arquiteturas são denominadas tiled architectures. Como exemplos, pode-se citar a WaveScalar [Swanson et al. 2003] [Swanson et al. 2006], TRIPS [Sankaralingam et al. 2003] [Burger et al. 2004], Smart Memories [Mai et al. 2000], e Raw [Lee et al. 1998].

Em todas as arquiteturas mencionadas, uma vantagem em comum é a excelente escalabilidade. Tanto a TRIPS quanto a Raw têm um bom desempenho em uma ampla variedade de tarefas com diferentes tipos de paralelismo. Uma característica da Raw é não utilizar reconfiguração de hardware; já as arquiteturas TRIPS e Smart Memories precisam de hardware reconfigurável, e, por isso, podem se adaptar a uma ampla variedade de tarefas para o aumento do desempenho.

Outra vantagem dessas arquiteturas é a incorporação de conceitos de processamento a fluxo de dados. No entanto, as arquiteturas Raw, Smart Memories e TRIPS, apesar de expandirem de maneira notável o paradigma da arquitetura de von Neumann, ainda dependem de um program counter para o seqüenciamento da execução do programa e acesso à memória, o que limita o paralelismo. Uma vantagem da arquitetura WaveScalar é o completo abandono do conceito de program counter, sendo uma arquitetura de fato a fluxo de dados. Por esse motivo, esta arquitetura será descrita com mais detalhes a seguir.

\subsubsection{Arquitetura WaveScalar}

A arquitetura WaveScalar é baseada no modelo a fluxo de dados dinâmico, incorporando o conceito de tagged-tokens. Assim como nas arquiteturas tradicionais, seus programas estão na forma de um grafo. Esta arquitetura difere das tradicionais por suportar, de maneira eficiente, a 
semântica de memória encontrada no modelo clássico de von Neumann. Por isso, a arquitetura WaveScalar não necessita de uma linguagem de programação especial e limitada, podendo executar programas escritos usando linguagens imperativas como $\mathrm{C}$ e $\mathrm{C}++$. Sendo uma arquitetura a fluxo de dados, ela completamente abandona o conceito de program counters e de execução linear característicos da arquitetura de von Neumann.

$\mathrm{Na}$ arquitetura WaveScalar, o núcleo de execução é formado por elementos de processamento (PE), cujo diagrama de blocos é ilustrado pela Figura 2.12. Do ponto de vista do programador, o modelo de execução provê um PE para cada instrução estática em uma aplicação binária. Como isso não é eficiente ou prático, na realidade o processador contém uma pequena quantidade de elementos de processamento e liga as instruções a eles de forma dinâmica. Um PE contém toda a lógica necessária para a execução a fluxo de dados, tendo uma interface de entrada que recebe tokens e informações que os associam a suas respectivas instruções. Os tokens são armazenados em uma tabela de matching, que é implementada em uma cache pequena nãoassociativa. Uma vez que todos os tokens para uma instrução foram recebidos, a instrução pode ser agendada para execução, que será feita por meio da ULA, que enviará os resultados para a interface externa da rede de comunicação, que os enviam a PEs consumidores ou para um buffer, próprio da arquitetura do WaveScalar, e que possibilita a execução de linguagens imperativas.

Como mostra a Figura 2.12, os elementos de processamento na arquitetura WaveScalar foram implementados com cinco estágios de pipeline, que são descritos a seguir:

- Entrada (input): Mensagens de operandos chegam ao PE por meio de outro PE ou do próprio.

- Combinação (match): Os operandos entram na tabela de matching, que determina quais instruções estão prontas para disparar.

- Envio (dispatch): O PE escolhe uma instrução da fila de execução, lê seus operandos pela tabela de matching, e os envio para o estágio "execução".

- Execução (execute): Executa a instrução e envia os resultados para a fila de saída e/ou para a rede de comunicação local. 
- Saída (output): Uma saída de instrução é enviada para as instruções consumidoras por meio da rede de comunicação interna. Consumidores podem ser outros PEs ou o próprio.

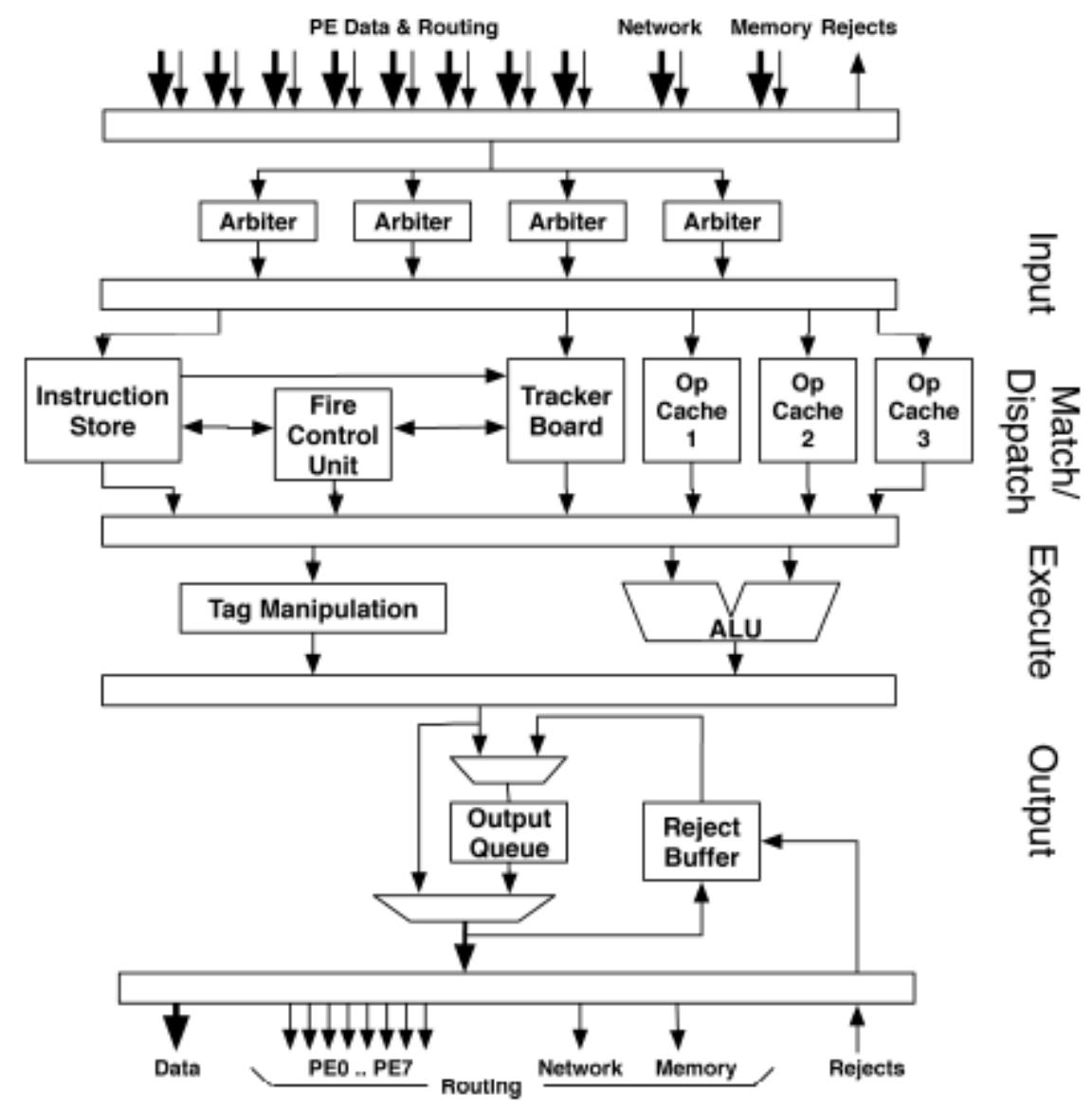

\section{Figura 2.12 - WaveScalar: Diagrama de blocos de um elemento de processamento} [Swanson et al. 2006].

Nos PEs, o processo de inserção de instruções é critico para um bom desempenho. Em Mercaldi [2005], o algoritmo é descrito em detalhes. A Figura 2.13 mostra como um programa pode ser mapeado em um processador WaveScalar para a execução. Na figura à esquerda, tem-se um código simples em C. O grafo a fluxo de dados do WaveScalar é mostrado na figura central. Já a figura à direita ilustra o mapeamento, no processador WaveScalar, do grafo em dois domínios com oito PEs em cada um. 

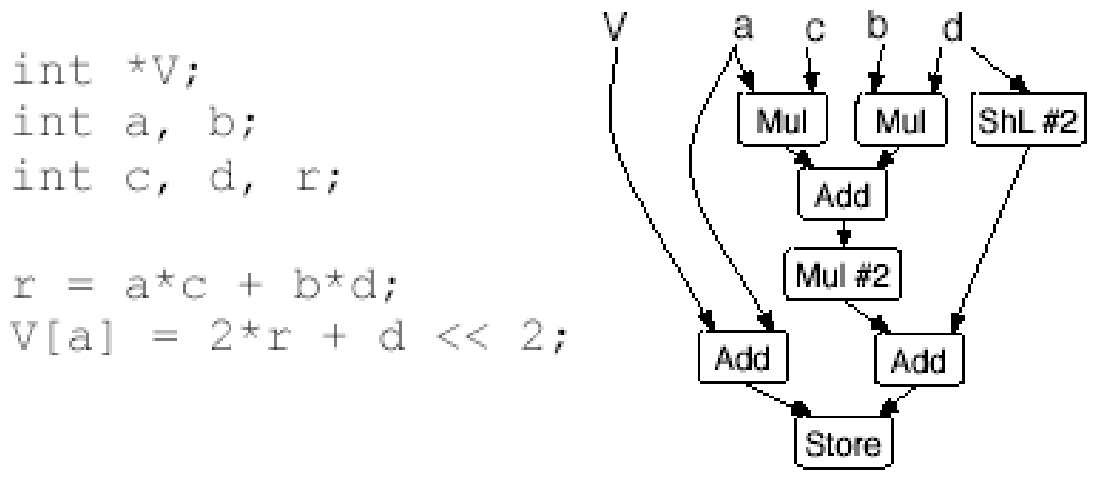

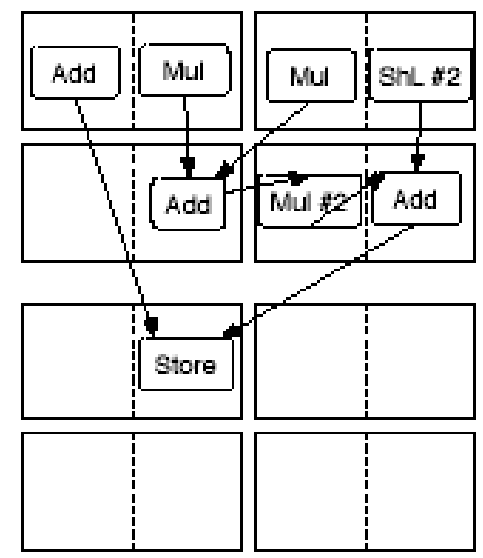

Figura 2.13 - Exemplo de mapeamento de código fonte no processador WaveScalar [Swanson et al. 2006].

Para que haja redução dos custos de comunicação, todos os componentes, isto é, PEs, buffers de armazenamento (store-buffers), e caches de dados (data-caches), são conectados através de uma estrutura hierárquica de interconexão, mostrada pela Figura 2.14. Conjuntos de PEs primeiramente são agrupados em pods que compartilham resultados das ULAs por meio de uma rede de desvio comum. Os pods são agrupados em domínios (domains), nos quais os PEs se comunicam por meio de barramentos em pipeline. Quatro domínios formam um cluster, que contém o hardware da memória, no buffer de armazenamento, um switch de comunicação, e uma cache L1. Um programa WaveScalar pode ser executado se há um cluster combinado com uma cache L2 e uma memória principal tradicional, mas, para a construção de máquinas com alto desempenho, é necessário agrupar vários clusters em uma rede intra-chip.

Apesar de ser uma arquitetura promissora, conseguindo, em benchmarks da SPEC, desempenho duas a sete vezes maior, quando comparada com arquiteturas superescalares de alto desempenho [Swanson et al. 2003], a arquitetura WaveScalar ainda se encontra em seu estágio inicial de desenvolvimento, tendo ainda vários desafios aguardando solução, como, por exemplo, gerenciamento de interrupções, I/O, entre outros problemas relacionados a sistemas operacionais. 


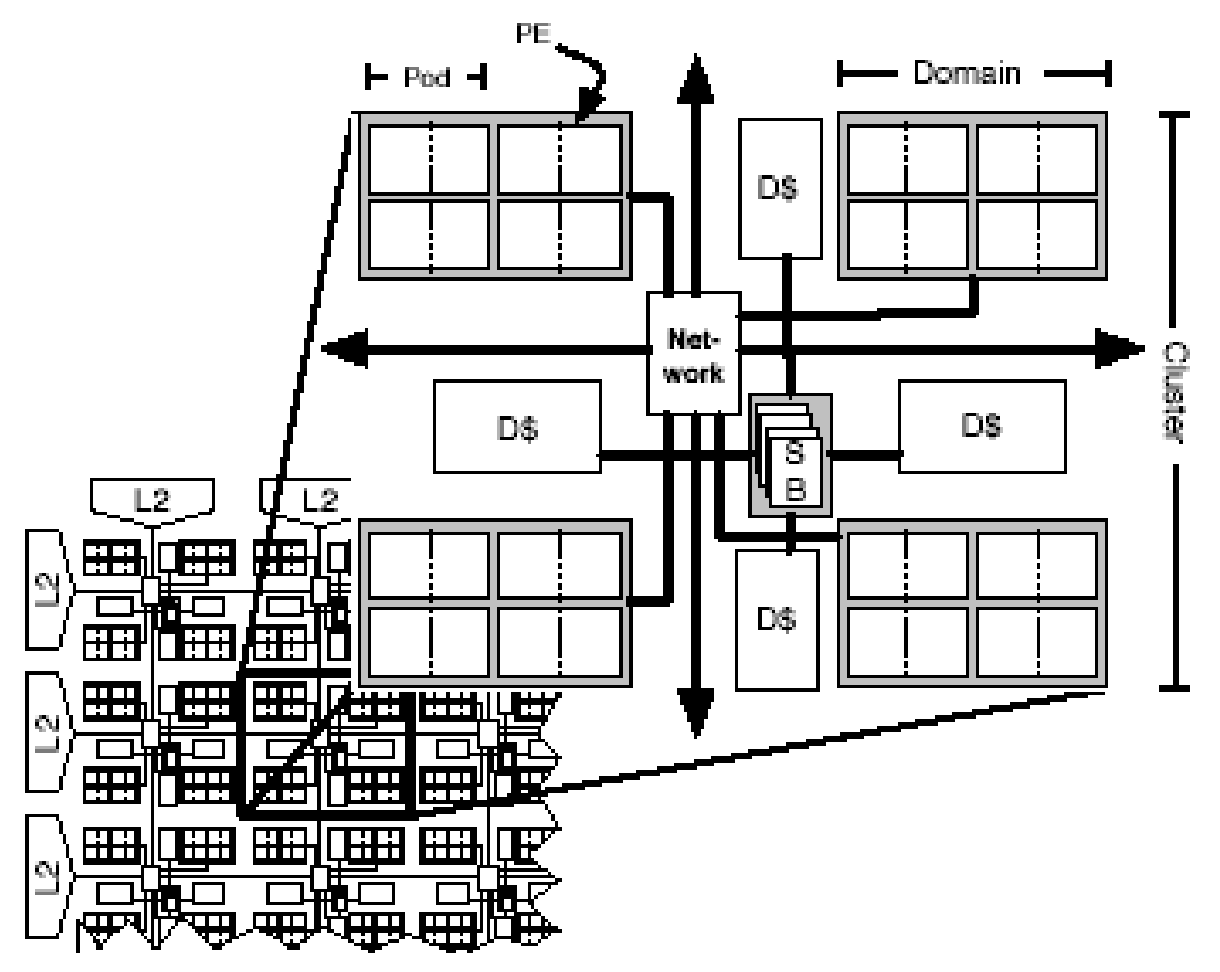

Figura 2.14 - Organização hierárquica da arquitetura WaveScalar: Processadores agrupados em um cluster [Swanson et al. 2006]. 


\section{ChipCflow - Conceitos Básicos}

Este trabalho é parte do projeto para o desenvolvimento da ferramenta ChipCflow, coordenada desde 2006 pelo Prof. Dr. Jorge Luiz e Silva, do ICMC - USP, sendo um projeto de arquitetura a fluxo de dados dinâmico em hardware reconfigurável. A ferramenta ChipCflow tem como objetivo a execução, em hardware, de algoritmos escritos em linguagens imperativas de alto-nível, como $\mathrm{C}$, aproveitando os benefícios do modelo a fluxo de dados dinâmico e as características do hardware reconfigurável [Silva 2006] [Silva \& Marques 2006] [Lopes et al. 2006] [Astolfi \& Silva 2007] [Costa 2009] [Silva et al. 2009]. A Figura 3.1 descreve a seqüência de atividades para que uma aplicação seja executada na ferramenta ChipCflow.

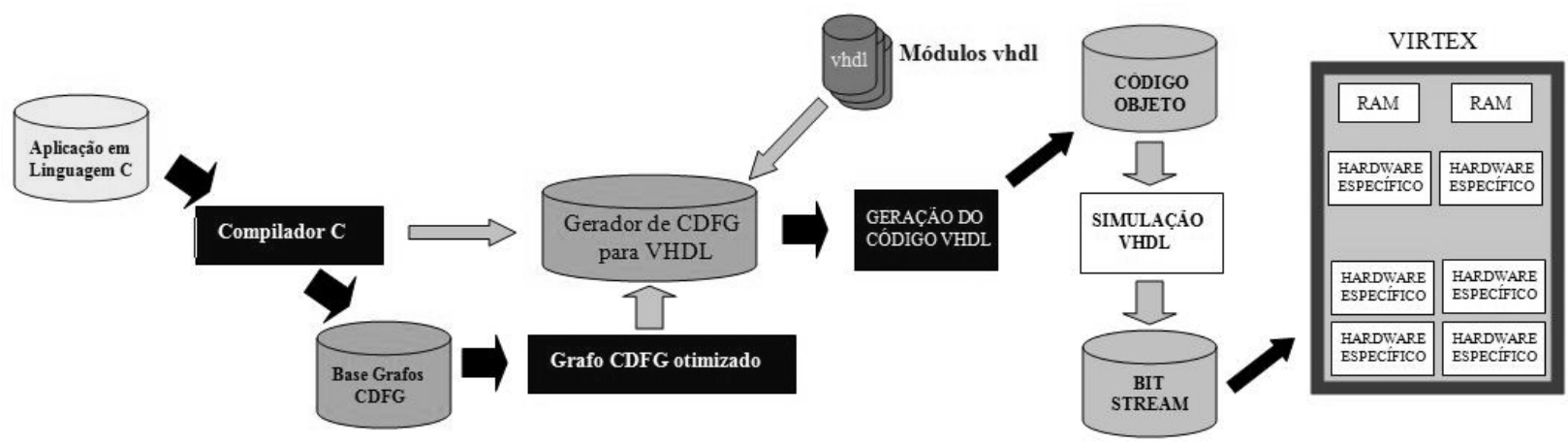

Figura 3.1 - Diagrama de fluxo da ferramenta ChipCflow. 
Inicialmente, extrai-se grafos CDFG a partir de programas de aplicação escritos em C, por meio de modelos utilizados em compiladores como Streams-C [Gokhale et al. 2000], Spark [Gupta et al. 2003], Impulse C [Pellerin \& Thibault 2005], ou o DK Design Suite [Celoxica URL]. Um grafo CDFG representa os operadores da linguagem $\mathrm{C}$ e chamadas de funções, além do fluxo de dados entre esses operadores. O grafo CDFG, que já estará otimizado, é convertido para a linguagem de descrição de hardware VHDL, tendo como base o conjunto de operadores proposto para o projeto, já implementados em VHDL [Astolfi \& Silva 2007]. O código VHDL é, então, sintetizado e simulado em uma ferramenta EDA comercial, sendo o bitstream gerado para ser executado direto no hardware.

O modelo a fluxo de dados proposto para a ferramenta ChipCflow segue a linha apresentada por Veen [1986], que é baseada em grafos contendo operadores interligados por arcos. Como mostrado no Capítulo 2, há dois tipos de modelos a fluxo de dados, o estático e o dinâmico. No primeiro, somente um token pode estar em um arco de cada vez; no segundo, mais que um pode estar no arco ao mesmo tempo. Como no projeto ChipCflow o modelo escolhido foi o dinâmico, pelas vantagens inerentes a esse modelo, foi necessário estabelecer um mecanismo para gerenciar a presença de vários tokens em um mesmo arco, que foi denominado modelo de instâncias, descrito a seguir.

\subsection{O Modelo de Instâncias}

No modelo de instâncias, quando um token chega a um operador, uma nova cópia desse operador é criada, para que esse token seja absorvido. Para a identificação dos tokens parceiros em um operador é necessário incorporar tags a eles. Dessa maneira, várias instâncias dos operadores podem ser criadas, cada uma esperando por seus dados parceiros, para posterior execução. Isso significa inserir um operador ou sub-grafo novo ao já existente, que representa uma nova instância.

Para a implementação do modelo em um grafo a fluxo de dados, foi necessária a criação de um padrão para inserção e remoção de sub-grafos. A Figura 3.2 ilustra um grafo T e o subgrafo F que será inserido no grafo original T. O modelo de inserção e remoção de grafos se baseia 
em uma estrutura de concatenação. Na Figura 3.2 (a), o grafo original $\mathrm{T}$ tem dois pontos de concatenação, que estão entre dois operadores, entre os quais o grafo F da Figura 3.2 (b) será concatenado. O resultado da concatenação é mostrado na Figura 3.3.

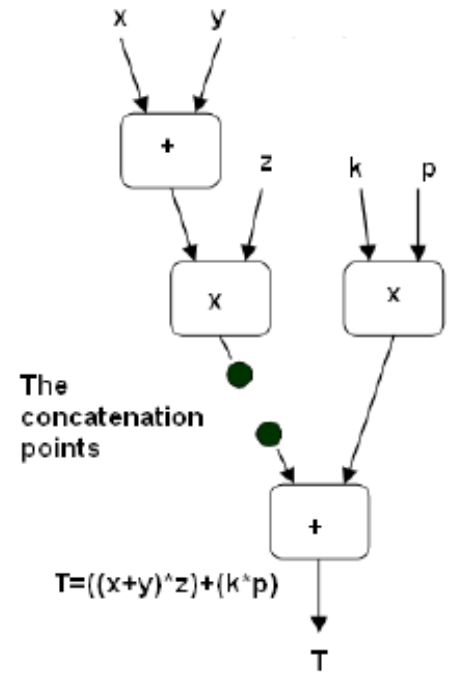

a) Graph $T$

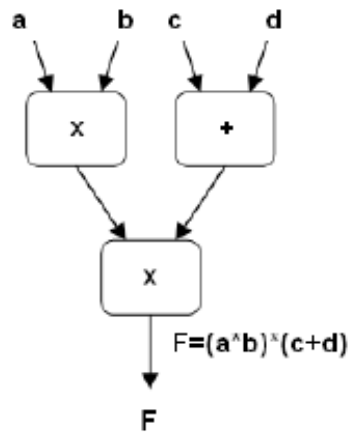

b) Sub-Graph F

Figura 3.2 - Sub-grafo F a ser incluído no grafo T [Silva 2006].

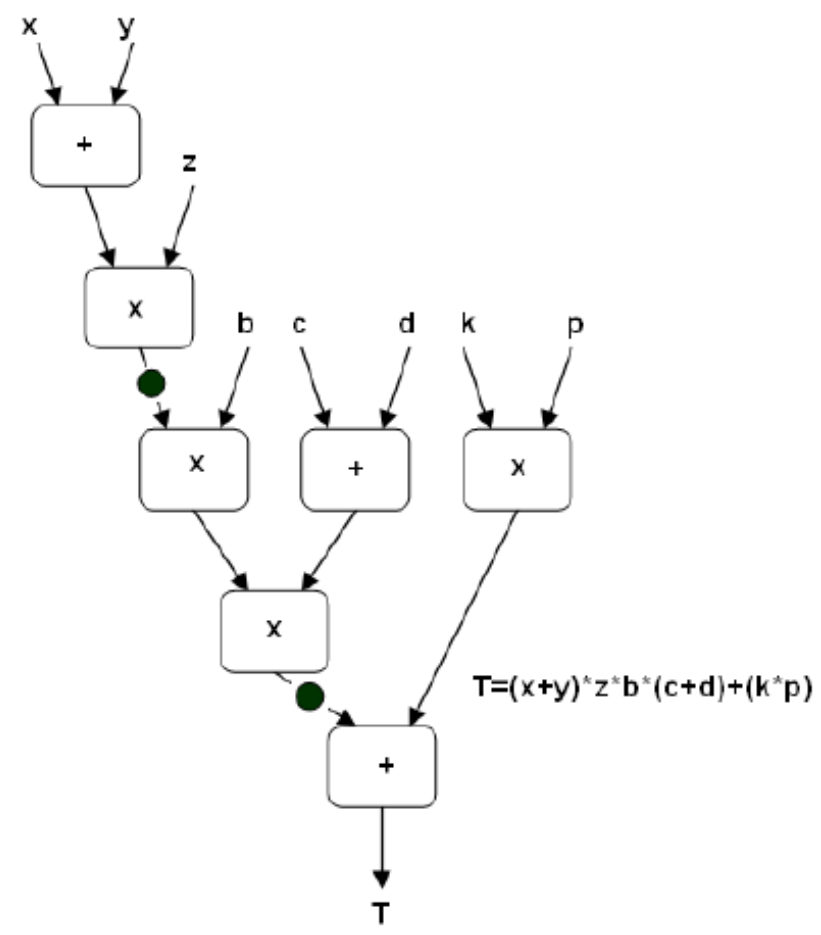

Figura 3.3 - Resultado da concatenação do sub-grafo F ao grafo T [Silva 2006]. 
Enquanto, para o funcionamento do modelo, em termos de grafo, basta apenas ligar os pontos de concatenação, no modelo prático é necessária a implementação de um protocolo de comunicação que informa "se" e "de onde" um token está vindo, e "se" e "para onde" um token está indo.

O processo de remoção de sub-grafos é semelhante ao processo de inserção já descrito, requerendo apenas a definição dos pontos onde a remoção deve ocorrer. Após a concatenação desses pontos, o sub-grafo entre eles é removido.

\subsection{A Estrutura Tagged-Token}

Como indicado no Capítulo 2, o conceito de tagged-tokens está associado ao modelo a fluxo de dados dinâmico. Tagged-tokens são utilizados para identificar tokens que pertencem a uma mesma instância. Isso é feito colocando-se, em cada token, uma etiqueta (tag) que identifica a que instância pertence o nó ao qual ele é direcionado. Os nós dessa estrutura têm uma regra de habilitação que especifica que o nó está habilitado se cada arco de entrada contém um token com tags idênticas. A identificação dos tokens, a partir das tags de cada token, requer uma estrutura de acoplamento de dados (matching), que será inserida em cada instância dos operadores para que a busca seja feita simultaneamente em cada uma delas [Silva 2006] [Silva \& Marques 2006].

Um exemplo de aplicação que caracteriza o uso de tagged-tokens é o laço expresso pela seguinte instrução for:

$$
\begin{aligned}
& z=0 ; \\
& \text { for }(i=0 ; i<N ; i++) \\
& \qquad z=z+(x \star y) ;
\end{aligned}
$$

O operador de multiplicação (“*”), utilizado na expressão $z=z+(x * y)$, fará parte de um grafo a fluxo de dados, podendo receber $\mathrm{N}$ dados diferentes e em tempos diferentes pelos arcos representando " $x$ " e "y". Cada vez que existir um novo dado " $x$ ", e seu correspondente " $y$ " ainda 
não estiver presente, ou vice-versa, uma nova instância do operador “*” deverá ser utilizada, esperando a chegada do seu par correspondente.

A utilização das instâncias no grafo a fluxo de dados se assemelha ao modelo de concatenação. Um sub-grafo de um operador é concatenado tendo como pontos de concatenação os mesmos pontos, ou arcos, de entrada e saída do operador. No caso do exemplo anterior, novas instâncias do operador "*” são criadas, tendo como pontos de concatenação os arcos "z" e o resultado do operador "x $+y$ ". Quando uma instância do operador é executada, um resultado é gerado e enviado para ser utilizado por outras instâncias, e tão logo esse resultado seja confirmado como recebido no operador destino, aquela instância é eliminada, permitindo que outras instâncias sejam geradas. A Figura 3.4 ilustra várias instâncias de um operador "*” em um grafo a fluxo de dados dinâmico.

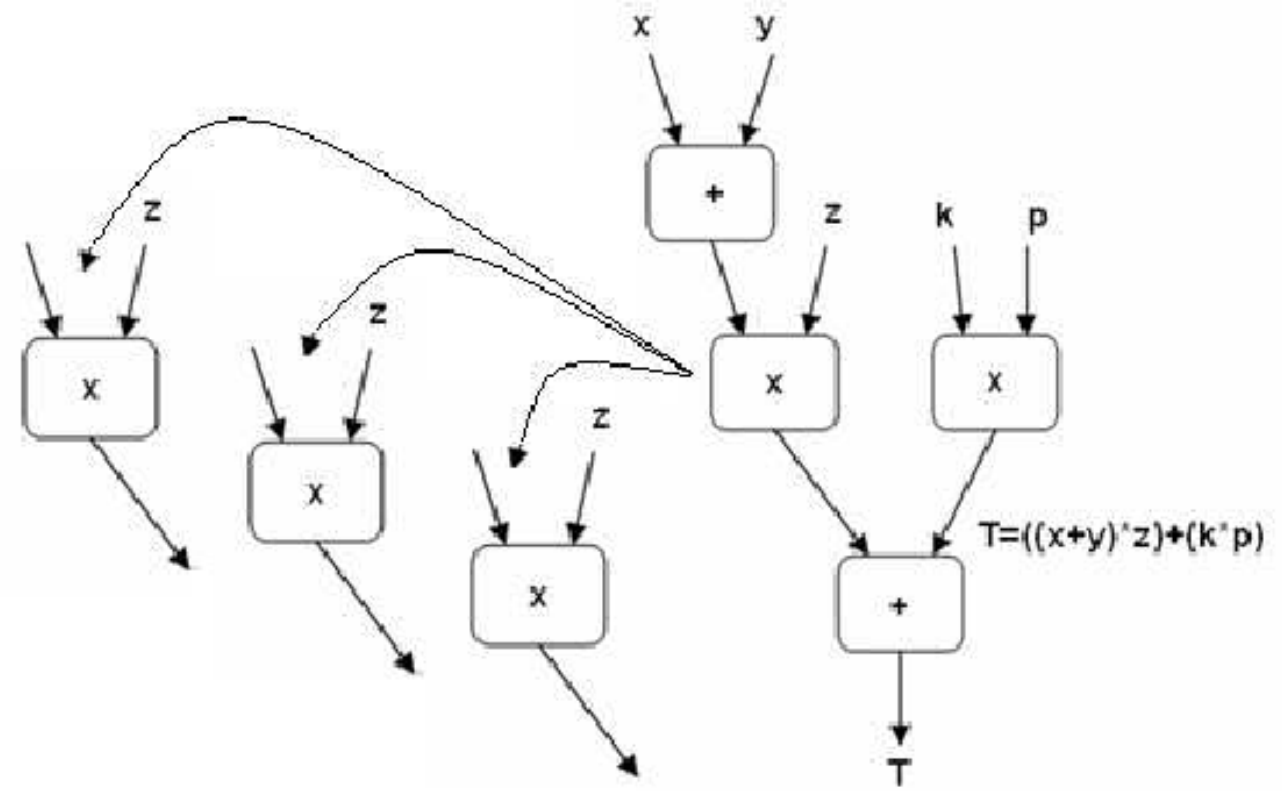

Figura 3.4 - Processo de criação de diferentes instâncias do operador “*””.

\subsection{Operadores utilizados no Modelo}

Os operadores utilizados no modelo são: Branch, Copy, Decider, Nondeterministic Merge, Operator, e Value Deterministic Merge. Esses operadores, ilustrados pela Figura 3.5, já foram 
descritos no Capítulo 2. Na Figura 3.6, pode-se ver exemplos de comandos em $\mathrm{C}$ convertidos para grafos que utilizam esses operadores.

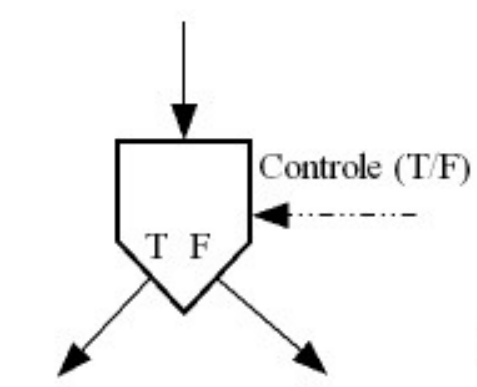

Branch

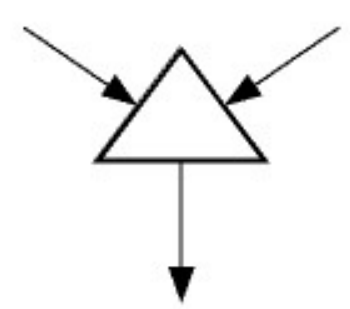

Nondeterministic Merge

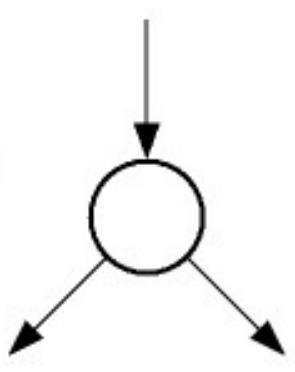

Copy

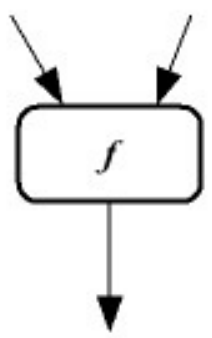

Operator

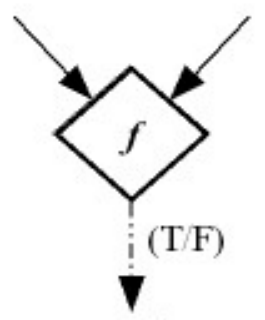

Decider

$(\mathrm{T} / \mathrm{F})$

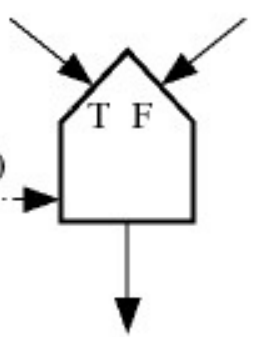

Value Deterministic Merge

Figura 3.5 - Operadores utilizados no modelo ChipCflow [Silva \& Marques 2006].

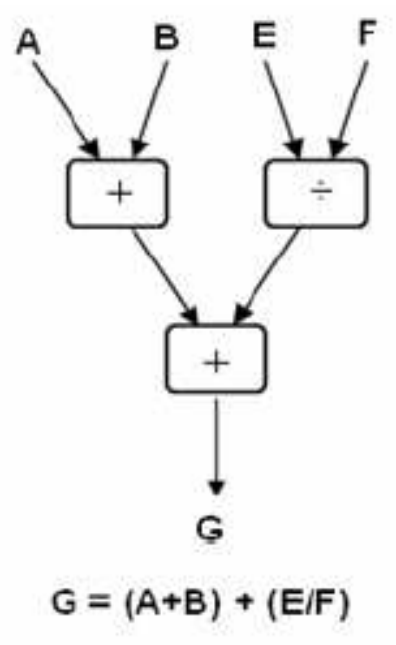

a) atribution command

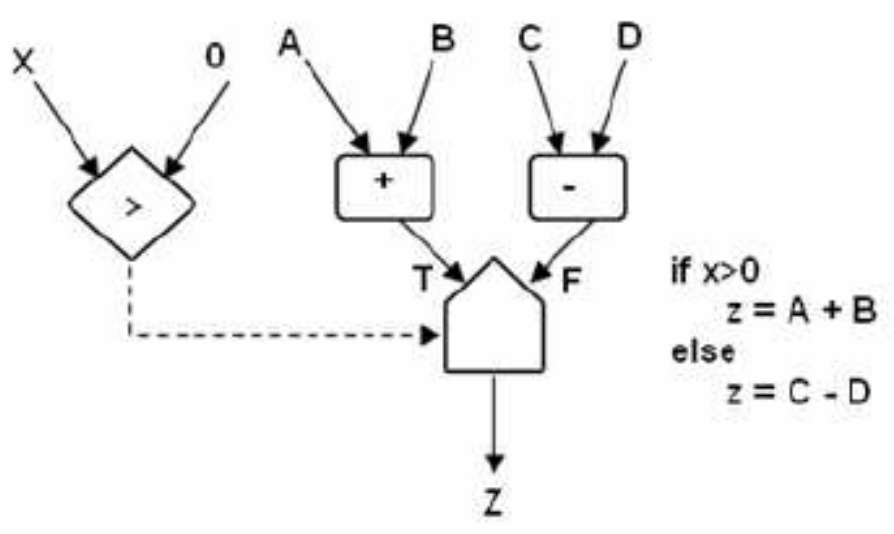

b) conditional command

Figura 3.6 - Em (a) está representado um grafo que representa um comando de atribuição; em (b) está representado o comando IF da linguagem C [Silva \& Marques 2006]. 


\section{4}

\section{Computação}

\section{Reconfigurável e FPGAs}

Antes do surgimento dos FPGAs, existiam duas maneiras de executar algoritmos em hardware. Uma delas baseia-se na utilização de circuitos digitais integrados especialmente projetados para alguma finalidade, os ASIC (Application Specific Integrated Circuits). Como qualquer circuito integrado, um ASIC tem sua lógica interna invariável fabricada sobre a superfície de um substrato de material semicondutor. Como eles são projetados para executar uma tarefa específica, as vantagens dos ASICs são o desempenho e a eficiência, tendo como restrição, porém, a ausência de flexibilidade e impossibilidade de posterior modificação ou otimização.

Outra maneira de se executar algoritmos é utilizando microprocessadores programados por software, nos quais a computação é feita utilizando-se um conjunto de instruções, o que permite a alteração da funcionalidade do sistema sem a alteração do hardware, provendo maior flexibilidade. Essa flexibilidade, entretanto, tem como custo o alto overhead de, por exemplo, tempo e potência consumida, que causa um decréscimo do desempenho.

Os sistemas de computação reconfiguráveis, ao contrário dos hardwares padrão de aplicação específica (ASIC), têm como característica fundamental o fato de poderem ser alterados durante seu ciclo de vida, sendo capazes, portanto, de combinar a especialização e 
desempenho do hardware com uma flexibilidade ainda maior que a encontrada pelo software, que está limitado pelas arquiteturas dos microprocessadores [Compton \& Hauck 2002]. No caso do hardware reconfigurável, é possível haver arquiteturas adaptadas às aplicações. A computação reconfigurável surgiu, portanto, como um elemento intermediário entre os ASIC e os microprocessadores, combinando flexibilidade e desempenho, consumindo, porém, mais energia que os circuitos integrados de aplicação específica (ASICs) quando desempenham a mesma função [Tuan \& Lai 2003].

Nos últimos anos, houve um grande avanço na computação reconfigurável, o que se deve às tecnologias para FPGAs (Field-Programmable Gate Arrays) desenvolvidas em meados da década de 1980. Em princípio, os FPGAs surgiram para a prototipacão rápida de circuitos, além de ser um elemento intermediário entre os processadores de propósito geral (General Purpose Processor - GPP), e os ASIC [Compton \& Hauck 2002]. Atualmente, FPGAs são capazes de desempenhar cálculos computacionais complexos em um único chip, ao ponto de muitos serem fabricados como sistemas completos em chip programável (System on Programmable Chip SoPC), que podem conter o equivalente a mais de dez milhões de portas lógicas.

Estima-se que, em 2010, os FPGAs estejam utilizando transistores de tecnologia de $32 \mathrm{~nm}$, possibilitando a incorporação de mais de cinco bilhões de transistores em uma única pastilha de FPGA [Bolsens 2006] [Wilson 2008]. Graças a sua flexibilidade, capacidade e desempenho, esses dispositivos criaram novos caminhos na computação, formando a base da computação reconfigurável.

\subsection{Estrutura Básica dos FPGAs}

Um FPGA é um dispositivo semicondutor formado por um arranjo de células ou blocos lógicos configuráveis contidos em um único chip, cujas funcionalidades são determinadas por múltiplos bits de configuração. Essas células lógicas têm capacidade computacional para implementar funções lógicas ou realizar comutação que permite a comunicação entre as células. A estrutura básica de um FPGA é mostrada na Figura 4.1. Pode-se identificar, na figura, três tipos estruturais, que serão descritos a seguir. 


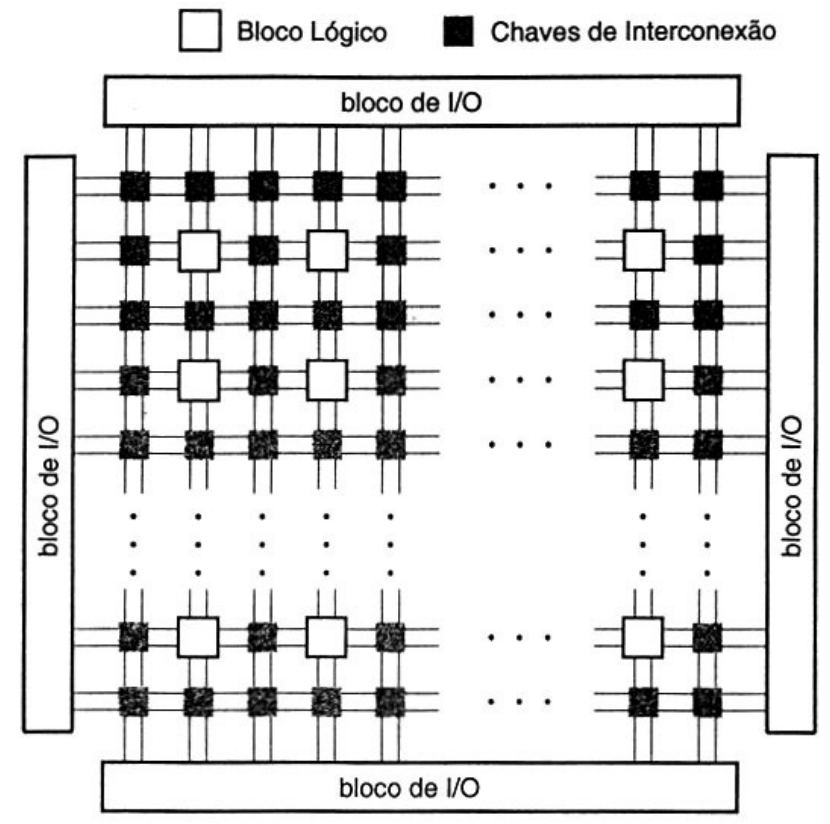

Figura 4.1 - Estrutura básica de um FPGA, adaptado de Brown \& Vranesic [2005].

- Blocos Lógicos (CLB): Circuitos formados por um ou mais flip-flops, utilizando lógica combinacional. Os elementos lógicos em um FPGA são construídos a partir desses blocos. Um exemplo de bloco lógico, com uma LUT (Look-Up Table) de quatro entradas e um flip-flop D, é mostrado pela Figura 4.2. Uma LUT de N entradas é basicamente uma memória que, quando programada de modo apropriado, pode computar qualquer função com até $\mathrm{N}$ entradas, permitindo, assim, que qualquer função seja implementada de até $\mathrm{N}$ variáveis booleanas, ao prover lógica genérica. Os flip-flops, por sua vez, são necessários em situações nas quais clocks são necessários, como, por exemplo, pipelining, registradores, máquinas de estados finitas, entre outras.

- Blocos de I/O (IOB): Circuitos que realizam a interface entre a lógica interna e os pinos de entrada e saída da FPGA. 
- Chaves de Interconexão (switch matrix): Utilizadas para a criação de interconexões arbitrárias entre os blocos lógicos e de $\mathrm{I} / \mathrm{O}$ no sistema. O processo de escolha das interconexões é chamado roteamento.

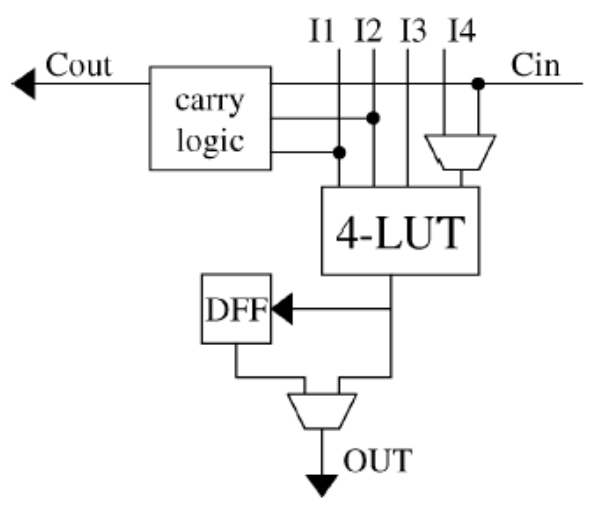

Figura 4.2 - Um bloco lógico básico de um FPGA [Compton \& Hauck 2002].

\subsection{Construção de Lógica em FPGA}

O processo de construção de aplicações em FPGA, ilustrado na Figura 4.3, tem suas etapas detalhadas a seguir [Nascimento et al. 2006].

- Especificação: O comportamento da FPGA é definido pelo projetista por meio da utilização de linguagens de descrição de hardware (HDL), em geral Verilog ou VHDL. Alternativamente, pode ser utilizada uma forma gráfica, com blocos esquemáticos.

- Síntese Lógica: Após a definição do projeto inicial, inicia-se o processo de síntese lógica, que consiste na geração de uma netlist do circuito, que descreve a interconexão dos componentes digitais em baixo nível, como portas lógicas, flip-flops, e latches. Durante essa etapa, é feita otimização dos circuitos para melhoria da eficiência.

- Mapeamento Tecnológico: Tem como objetivo o mapeamento dos componentes da netlist para os blocos básicos da FPGA (CLBs e IOBs), descritos em uma biblioteca. Este processo é feito pela decomposição da netlist em sub-circuitos que podem ser 
implementados dentro dos CLBs e pela associação dos sinais de entrada e saída para os IOBs. Esse processo é feito de tal maneira que os recursos internos dos CLBs sejam utilizados da maneira mais efíciente possível, minimizando o número de CLBs necessários.

- Place \& Router: O placer determina a posição dentro do FPGA que cada bloco lógico deve ocupar, tendo como objetivo a facilitação do processo de roteamento das conexões e a minimização do atraso de comunicação. Já o router implementa todas as conexões entre blocos e pinos de entrada e saída.

- Geração do Bitstream: Após o roteamento, torna-se disponível, como saída, uma descrição de todas as configurações necessárias para a programação do FPGA, que é convertida em um bistream utilizado para a programação do FPGA por meio de sua interface de configuração.

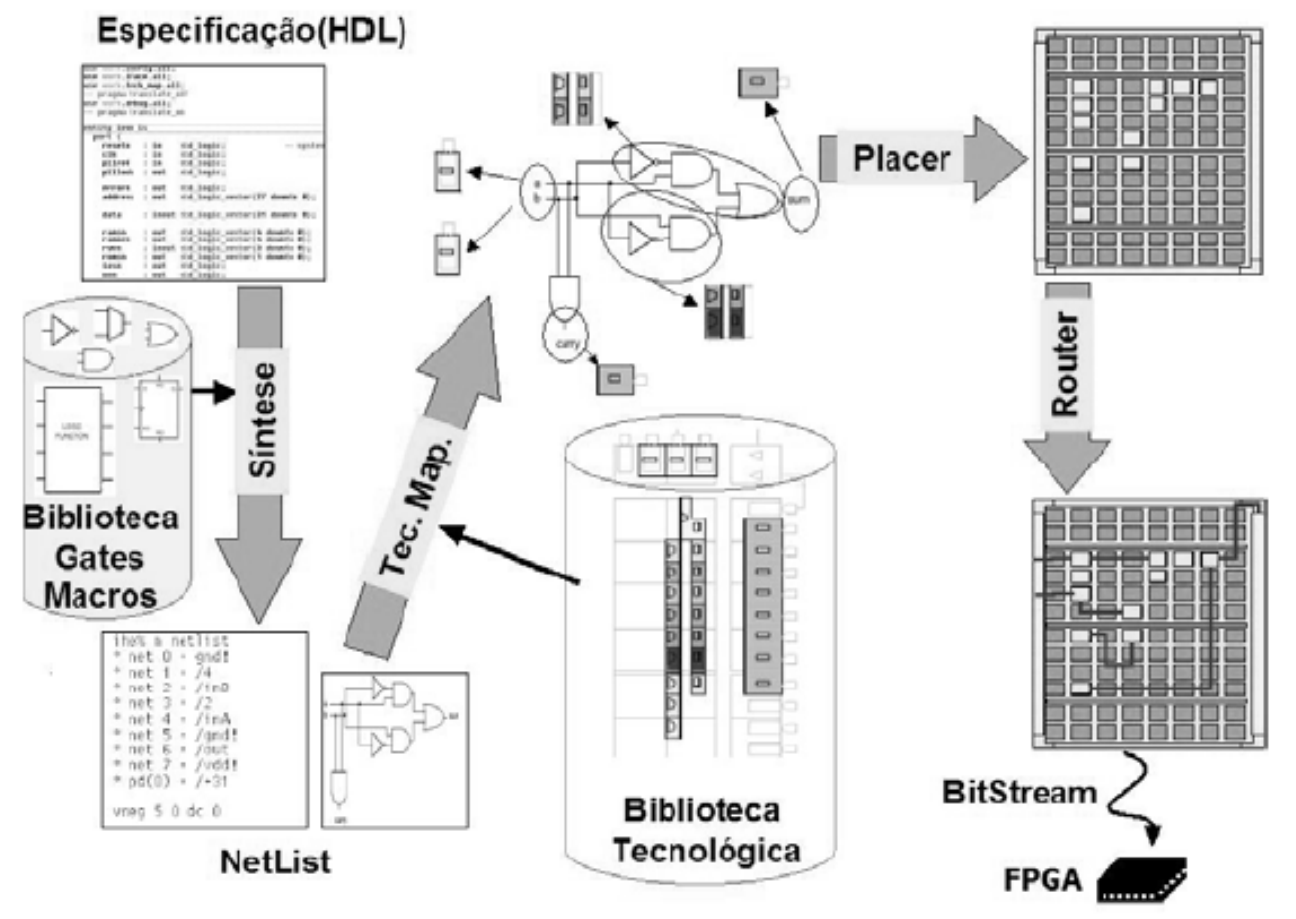

Figura 4.3 - Fluxo de projeto para construção de aplicações FPGA [Nascimento et al. 2006]. 
Nos FPGAs da Xilinx, faz-se uso da plataforma de software Integrated Software Environment (ISE) como ferramenta para o ambiente de projeto [Xilinx 2007d]. Essa ferramenta engloba as etapas necessárias para o desenvolvimento de uma aplicação em FPGA descritas nesta seção.

\subsection{Tecnologias de Configuração}

No que se refere à programação das FPGAs, existem várias maneiras de se implementar as chaves de interconexão [Ribeiro 2002]. Dentre elas, pode-se citar:

- Antifusíveis (antifuses): Originalmente um circuito aberto, quando programado forma um caminho de baixa resistência. Entre suas vantagens, destacam-se o tamanho reduzido, o que se reflete em um maior desempenho. Essa técnica, entretanto, não permite a reprogramação.

- Porta Flutuante: Nesse caso, a chave de interconexão é um transistor com gate flutuante. As maiores vantagens dessa técnica são a capacidade de reprogramação e de retenção de dados. Como desvantagem, destaca-se a maior complexidade, se comparada com a técnica baseada em antifusíveis. Os circuitos atualmente se baseiam principalmente em flashes, que substituíram as EPROMs e EEPROMs.

- SRAM (Static Random Access Memory): A chave de interconexão é um transistor de passagem controlado pelo estado de um bit de SRAM. Essa memória é volátil, necessitando, portanto, ser configurada quando o chip é ligado, o que implica a existência, no FPGA, de uma memória externa do tipo PROM, EPROM, EEPROM, disco magnético, ou flash, sendo essa última a tecnologia mais atual. Uma desvantagem da tecnologia SRAM é a ocupação de espaço maior no chip. Como vantagem, destaca-se a rapidez da reprogramação. 


\section{Reconfiguração Dinâmica em FPGAs}

Apesar dos FPGAs sempre terem sido programáveis, no que diz respeito à reconfiguração, era necessário interromper seu funcionamento para que fosse feita a reconfiguração denominada estática, em tempo de compilação, ou offline. Esse tipo de reconfiguração, contudo, não explora todo o potencial que pode ser obtido sob a perspectiva da computação reconfigurável [Compton \& Hauck 2002]. Quando a reconfiguração em tempo de execução (run-time reconfiguration RTR), também conhecida como dinâmica, ativa, ou on-the-fly (OTF), tornou-se possível, era inicialmente restrita à reconfiguração completa do chip. Com o advento dos FPGAs com reconfiguração parcial em tempo de execução (PRTR), finalmente foi possível reconfigurar apenas parte do FPGA.

Como ilustra a Figura 5.1, os FPGAs podem ser classificados de acordo com sua configurabilidade. Este Capítulo dará ênfase às gerações de FPGAs que permitem reconfiguração parcial dinâmica. Serão apresentados FPGAs que representam o estado da arte em computação reconfigurável, em específico a família Virtex, da Xilinx. Em seguida, serão apresentadas as maneiras como a reconfiguração parcial é obtida nesses dispositivos. 


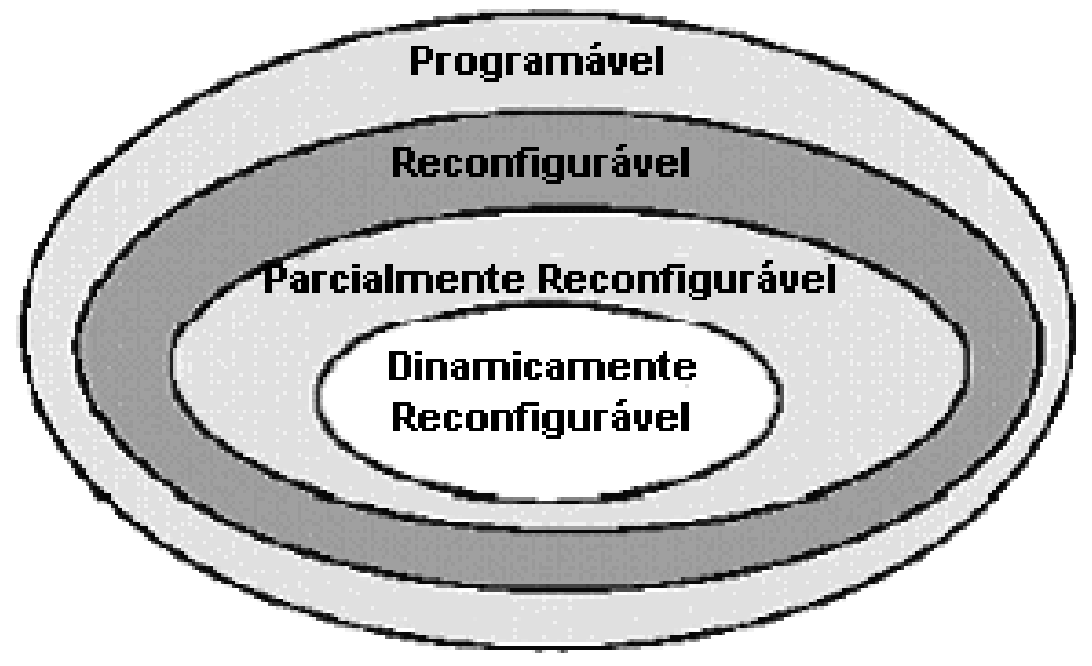

Figura 5.1 - Classes de configurabilidade dos FPGAs [Ribeiro 2002].

\subsection{Reconfiguração Dinâmica}

Um FPGA é classificado como dinamicamente reconfigurável se seus circuitos internos de armazenamento da configuração podem ser atualizados seletivamente, sem prejudicar o funcionamento da lógica restante em funcionamento. A reconfiguração dinâmica também é conhecida como reconfiguração em tempo de execução (RTR), ativa, in-circuit, ou on-the-fly (OTF) [Ribeiro 2002].

Pode-se citar como exemplo de utilização da RTR um aplicativo cujos procedimentos a serem acelerados pelo uso de hardware reconfigurável são muito numerosos ou complexos para serem carregadas simultaneamente no FPGA disponível. Para casos como esse, é interessante separar a aplicação em duas ou mais configurações que podem ocupar o hardware em tempos diferentes, como mostra a Figura 5.2 [Compton \& Hauck 2002]. Nesse caso, o FPGA sempre é reconfigurado em sua totalidade.

O exemplo anterior ilustra o conceito de "hardware virtual", característico dos FPGAs reconfiguráveis dinamicamente, cujo funcionamento é similar ao das memórias virtuais dos 
sistemas operacionais. A idéia básica desta técnica é aumentar virtualmente o tamanho do FPGA do ponto de vista dos aplicativos. O conceito de hardware virtual é, portanto, uma técnica efetiva e eficiente para o aumento da disponibilidade dos recursos de hardware, implementação de circuitos maiores, e redução dos custos, pela adoção de FPGAs menores, com uma manutenção satisfatória do desempenho [El-Araby et al. 2007].

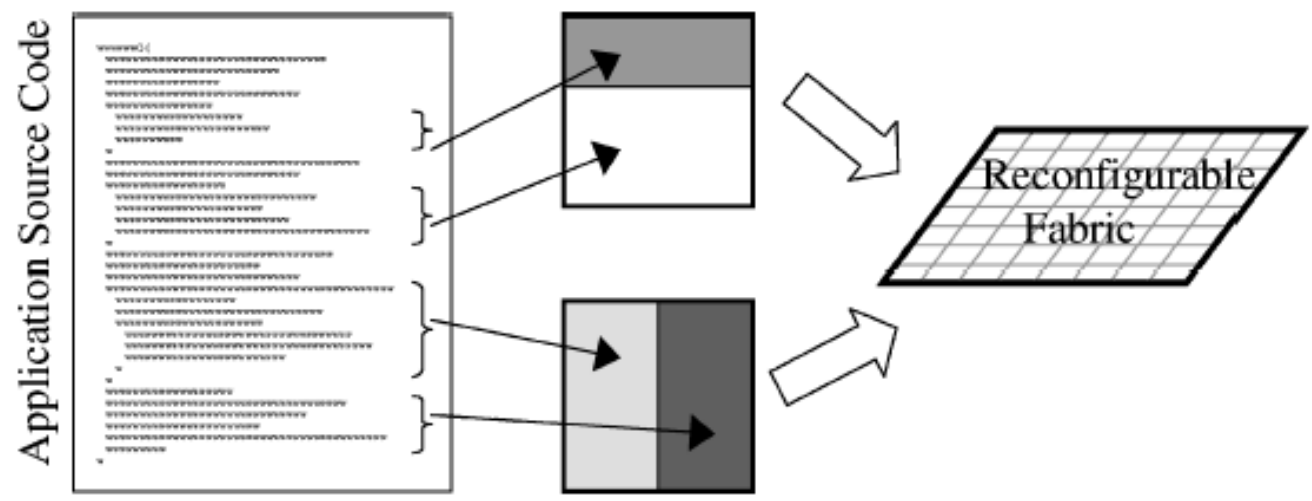

Figura 5.2 - Divisão de uma aplicação em duas configurações menores que podem ocupar o FPGA em tempos diferentes [Compton \& Hauck 2002].

Durante a execução de um único aplicativo, configurações podem ser inseridas e reinseridas no hardware. É possível que algumas configurações precisem de resultados obtidos por outras configurações. Nesse caso, a comunicação entre configurações pode ser feita através de registradores, nos quais o conteúdo permanecerá intacto entre as reconfigurações [Scalera \& Vazquez 1998].

Como ilustra a Figura 5.3, existem três tipos de dispositivos disponíveis pelos quais a reconfiguração dinâmica é possível [Skliarova \& Ferrari 2003]:

- Dispositivos de contexto único exigem uma reconfiguração completa para a alteração de qualquer bit de programação. A maioria dos FPGAs disponíveis no mercado pertence a esta classe. Existe uma técnica, entretanto, que possibilita a reprogramação parcial desse grupo de FPGAs, baseando-se em moldes - hardware templates. 
- Dispositivos de contexto múltiplo possuem várias camadas de bits de programação, estando em cada instante ativa apenas uma camada. A vantagem desta classe é a rápida alteração de contexto, na ordem de nanosegundos, enquanto a reconfiguração de um dispositivo de contexto único pode levar milissegundos [Compton \& Hauck 2002]. Além disso, é permitido que a reconfiguração, sempre total, se faça durante a execução da configuração ativa, possibilitando a reprogramação de um contexto enquanto outro está ativo.

- Dispositivos parcialmente reconfiguráveis permitem a reconfiguração de partes do FPGA sem a necessidade da reprogramação de todo o dispositivo. Por essa razão, a reconfiguração requer, geralmente, menos tempo que a reconfiguração total. Este tipo de reconfiguração será estudado com mais detalhes na Seção 5.1.1.

a)

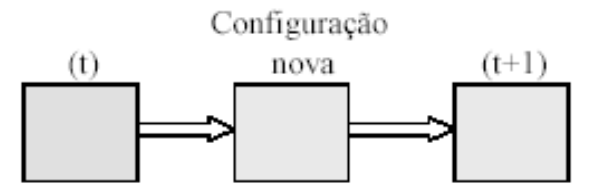

Configuração

b)

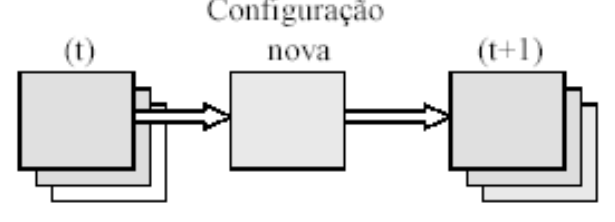

Configuração

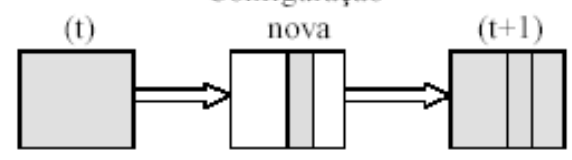

Dispositivo

de contexto único

Dispositivo de contexto múltiplo

Dispositivo parcialmente reconfigurável

Figura 5.3 - Os diferentes tipos de dispositivos reconfiguráveis [Skliarova \& Ferrari 2003].

Nas duas primeiras classes de dispositivos apresentadas (contexto único e múltiplo), o FPGA sempre deve ser totalmente reconfigurado, não havendo possibilidade de reconfiguração parcial, o que causa um overhead de tempo significativo nesses dispositivos. Devido ao acentuado tempo de reconfiguração, no caso de bitstreams muito grandes, a reconfiguração completa peca pela pouca praticidade [Moseley 2002]. Por exemplo, aplicativos em alguns sistemas desse tipo gastam de $25 \%$ a $98,5 \%$ de seu tempo de execução desempenhando 
reconfigurações [El-Araby et al. 2007]. Dessa maneira, arquiteturas parcialmente reconfiguráveis são mais apropriadas para a reconfiguração em tempo de execução quando há a necessidade de modificação de áreas pequenas do FPGA, não havendo necessidade de reprogramação de toda a lógica.

\subsubsection{Reconfiguração Parcial}

Em FPGAs parcialmente reconfiguráveis é necessário utilizar endereços para a especificação da localização da lógica, possibilitando, dessa maneira, a reconfiguração seletiva do FPGA. Uma desvantagem da reconfiguração parcial é a necessidade da transmissão dos endereços juntamente com os dados da reconfiguração, o que pode deixar a quantidade total de dados transferidos maior que o necessário para a reprogramação de um dispositivo de contexto único. Por isso, a reconfiguração total do FPGA parcialmente reconfigurável é mais demorada, se comparado com os FPGAs que só permitem esse tipo de reconfiguração. Entretanto, os FPGAs parcialmente reconfiguráveis são direcionados para aplicações nas quais o tamanho da configuração é pequeno o suficiente ao ponto de várias delas caberem no hardware simultaneamente. Dessa maneira, obtêm-se as vantagens da reconfiguração parcial, como aumento de desempenho devido ao menor tempo de configuração [Compton \& Hauck 2002] [Kao 2005].

Outra vantagem da PRTR é o compartilhamento de hardware. Uma vez que a reconfiguração parcial permite a execução de múltiplas aplicações em um único FPGA, o hardware é assim compartilhado. Isso possibilita a utilização de menos lógica, o que resulta em um menor consumo de energia, placas menores, e diminuição dos custos [Kao 2005].

A maior vantagem da reconfiguração parcial é, entretanto, a capacidade de intercalação de execução e configuração com menor prejuízo de desempenho. A Figura 5.4 [Ribeiro 2002] mostra um exemplo de reconfiguração dinâmica de um sistema composto de cinco circuitos ou tarefas. A tarefa A é uma função de entrada que alimenta as tarefas B, C, ou D, que alimentam, por sua vez, a tarefa E, que é a função de saída do sistema. As tarefas de entrada e saída são permanentes no FPGA, enquanto as outras se alternam sob o controle de um sinal de swap. 

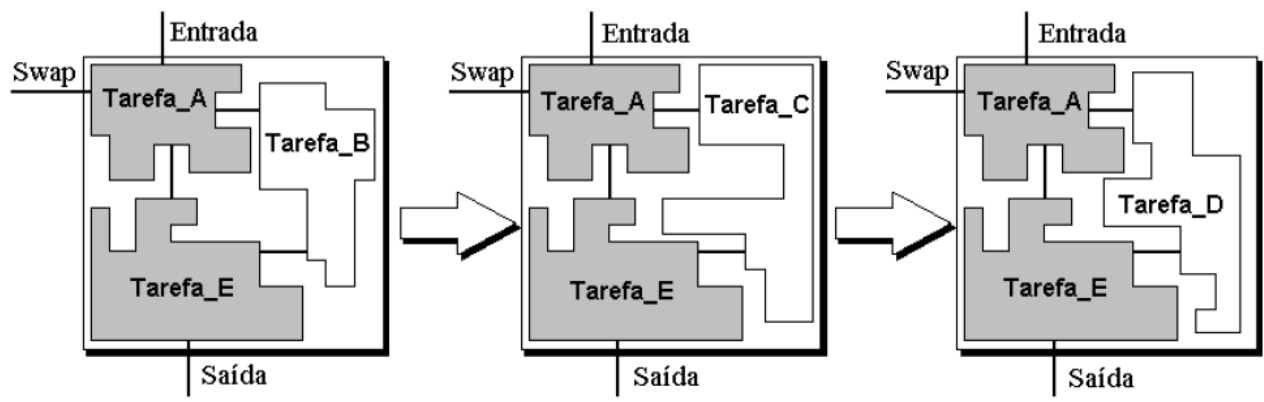

Figura 5.4 - Exemplo de reconfiguração dinâmica [Ribeiro 2002].

O gráfico da Figura 5.5 ilustra a execução, em função do tempo, das tarefas mostradas pela Figura 5.4, utilizando reconfiguração total. O gráfico da Figura 5.6 ilustra a execução das mesmas tarefas, dessa vez utilizando reconfiguração parcial dinâmica.

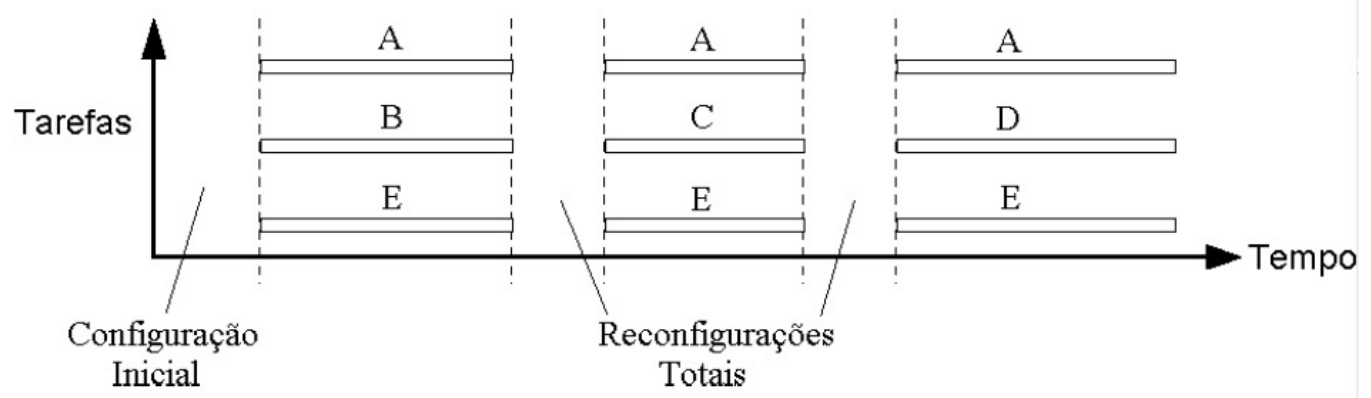

Figura 5.5 - Execução das tarefas com execução total [Ribeiro 2002].

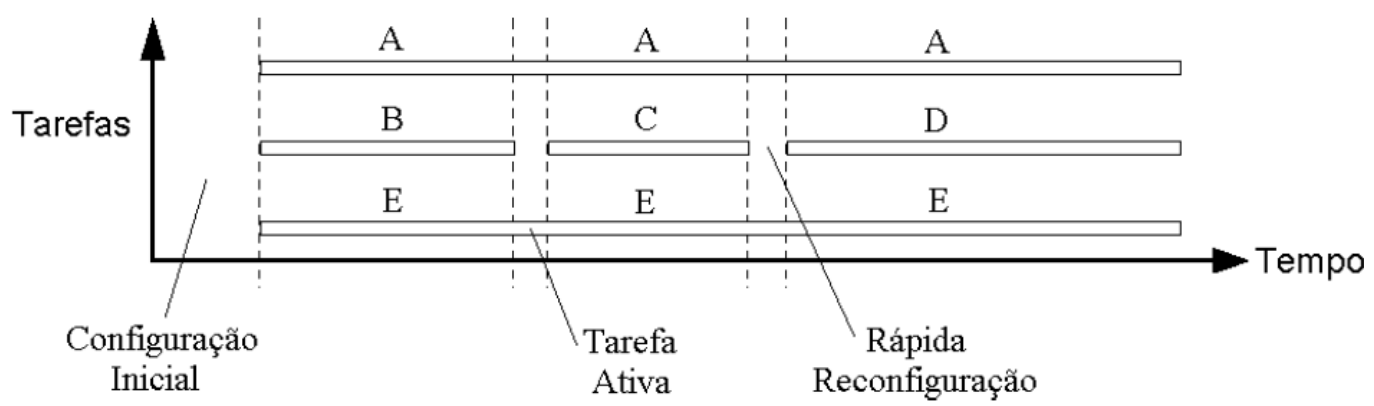

Figura 5.6 - Execução das tarefas com execução parcial [Ribeiro 2002]. 
Quanto à funcionalidade do dispositivo, a reconfiguração parcial pode ser dividida em "dinâmica" e "estática". Na primeira delas, também conhecida como "ativa", é possível a mudança de parte do FPGA enquanto o resto dele continua ativo e executando. Já na reconfiguração parcial estática, o FPGA fica em modo de espera ou inativo durante o processo de reconfiguração [El-Araby et al. 2007].

A reconfiguração parcial é disponibilizada por pelo menos dois fabricantes de FPGAs, sendo eles a Atmel [Atmel URL] e a Xilinx [Xilinx URL a]. Os FPGAs parcialmente reconfiguráveis fabricados pela Xilinx são os mais populares entre os desenvolvedores que utilizam essa técnica [El-Araby et al. 2007]. Depois da plataforma XC6200, que foi descontinuada, a partir da família Virtex todos os FPGAs da Xilinx podem ser parcialmente reconfiguráveis em tempo de execução, sendo essa reconfiguração ativa.

\subsection{Arquiteturas Reconfiguráveis}

A computação reconfigurável está se tornando muito importante para o estudo de arquiteturas de computadores e sistemas de software. As arquiteturas chamadas reconfiguráveis se caracterizam pelo uso de FPGAs ou outro hardware reconfigurável para a aceleração de algoritmos por meio do mapeamento de cálculos com alto custo computacional no substrato reconfigurável. Essa lógica reconfigurável está freqüentemente associada a um microprocessador de propósito geral, que é responsável pelo controle da lógica reconfigurável e pela execução em software das partes do programa que não podem ser aceleradas de maneira eficiente [Compton \& Hauck 2002].

Para algumas classes de tarefas, sistemas reconfiguráveis foram capazes de atingir desempenho muito superior ao obtido em computadores de propósito geral. De acordo com o objetivo principal que se pretende atingir, as tarefas podem ser dividas em três categorias, sendo elas descritas a seguir [Skliarova \& Ferrari 2003]. 
- Aceleração de tarefas computacionalmente intensivas - em geral, aplicada às implementações facilmente paralelizáveis, como, por exemplo, processamento de sinal e imagem (DSP).

- Emulação de hardware - utilizada, entre outras aplicações, para determinar se um circuito projetado está correto, além de seu desempenho.

- Hardware evolutivo - uma área com desenvolvimento recente, se comparada com as demais, utilizada para o desenvolvimento de circuitos eletrônicos com a ajuda de técnicas evolutivas.

Durante os últimos 20 anos, foram propostas muitas arquiteturas de sistemas reconfiguráveis, que diferem bastante em termos de mecanismos de interação dos componentes principais e dos modelos de programação e execução. A seguir, serão descritos alguns sistemas bem conhecidos que ilustram essas diferenças, não sendo necessariamente as arquiteturas mais atuais.

\subsubsection{DECPeRLe}

Trata-se de um dos primeiros trabalhos que se enquadram no paradigma de computação reconfigurável [Vuillemin et al. 1996]. O DECPeRLe-1 é um co-processador ligado ao barramento de E/S de um computador hospedeiro, que deve carregar um arquivo de configuração no PAM (Programmable Active Memories - nome da unidade de memória básica dessa arquitetura), possibilitando a leitura e escrita no PAM de uma maneira similar a uma memória, enquanto o PAM realiza o processamento dos dados entre as instruções de escrita e leitura.

O tipo de mecanismo de interação entre o hardware reconfigurável e o processador hospedeiro é, neste caso, o mais simples entre as arquiteturas reconfiguráveis. O hardware configurável representa uma unidade de processamento externa que se comunica raramente com o processador hospedeiro. Nesse tipo de arquitetura, o hardware programável efetua todas as computações de maneira independente e, no final, retorna ao processador principal os resultados, com a desvantagem da existência de um alto overhead de comunicação, devido à vazão e a latência entre os componentes. 
Como mostra a Figura 5.7, o co-processador foi implementado em uma placa contendo 16 FPGAs XC3090 da Xilinx, organizadas em uma "matriz" de dimensões 4x4. Cada FPGA tem ligações diretas com seus vizinhos. Há, além disso, um barramento comum. O DECPeRLe também possui 4 banco de memórias, totalizando 4 MBs. Quando utilizam os bancos de memória as aplicações devem funcionar a uma freqüência de aproximadamente $25 \mathrm{MHz}$; caso contrário, as aplicações podem funcionar a uma freqüência maior. No DECPeRLe há quatro conectores, sendo três necessários para o estabelecimento de conexão com dispositivos externos e um quarto para o estabelecimento da interface com o processador hospedeiro. Para a descrição dos circuitos, foi escolhida a linguagem $\mathrm{C}++$ complementada com uma biblioteca proprietária.

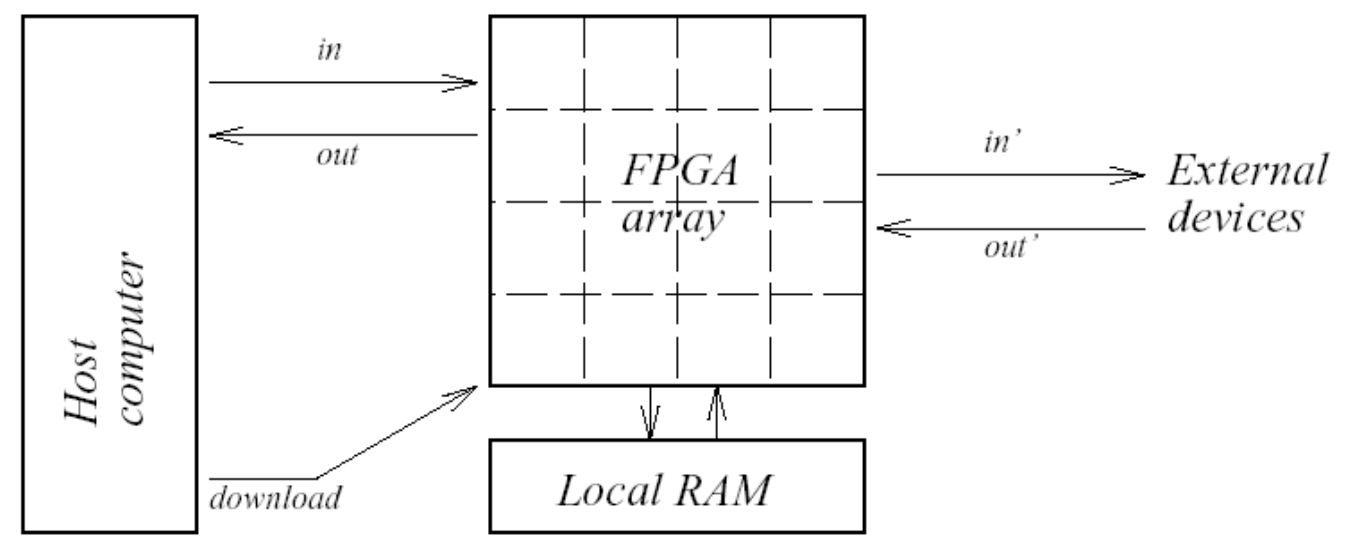

Figura 5.7 - Arquitetura do DECPeRLe [Vuillemin et al. 1996].

\subsubsection{Chimaera}

Em algumas arquiteturas, como a Chimaera [Hauck et al. 2004], tanto o hardware reconfigurável quanto o processador hospedeiro residem na mesma pastilha de silício, e são interligados fortemente. Neste caso, o hardware reconfigurável é usado para a implementação de unidades funcionais reconfiguráveis dentro do processador hospedeiro. Este modelo permite estender a programação tradicional com a capacidade de inserção de instruções personalizadas. Outra vantagem é o baixo overhead de comunicação, devido à baixa latência.

O sistema Chimaera caracteriza-se por ser parcialmente reconfigurável em tempo de execução. Ao contrário de outros sistemas semelhantes, que possuem processador e lógica 
configurável fortemente ligados, como o PRISC [Razdan \& Smith 1994], o Chimaera permite que o processador e a lógica executem simultaneamente.

Neste sistema, como ilustra a Figura 5.8, o componente principal é um array reconfigurável, onde as computações são realizadas. O array é composto por linhas de células lógicas entre as quais passam canais de encaminhamento horizontais. O array obtém as suas entradas diretamente dos registradores do processador hospedeiro. Cada linha do array reconfigurável tem como correspondente uma posição em uma memória CAM, que indica quais as instruções que já concluíram sua execução. A CAM analisa todas as instruções a serem executadas, recolhendo apenas aquelas que devem ser processadas pelo array reconfigurável. Se a instrução lida já está presente no array, o resultado de sua execução é enviado a um registro. Caso contrário, o processador é parado enquanto a lógica de controle carrega a instrução adequada da memória para o array reconfigurável.

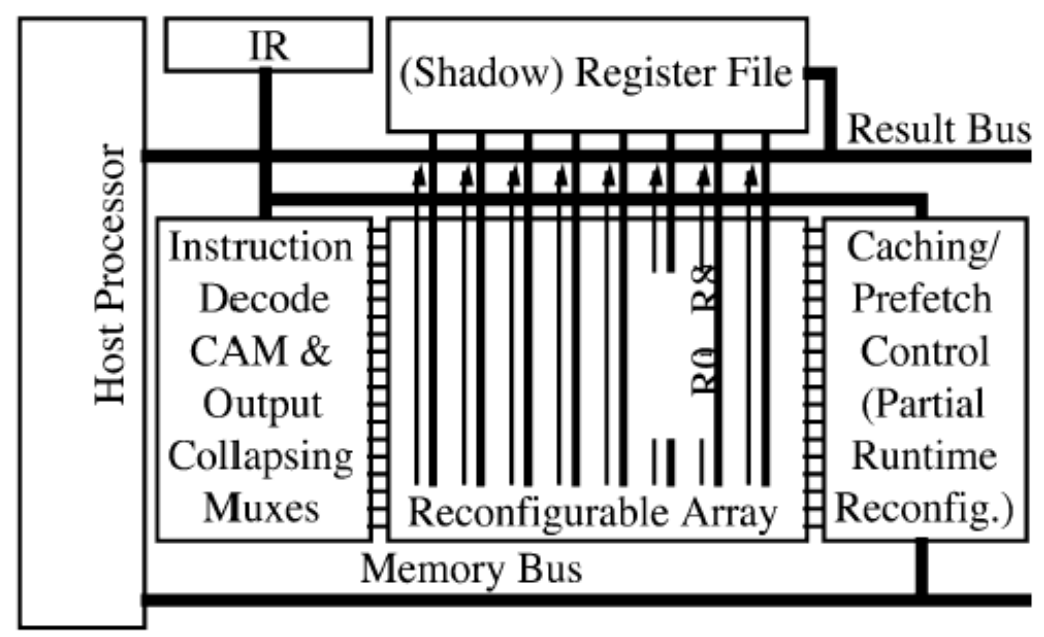

Figura 5.8 - Arquitetura do Chimaera [Hauck et al. 2004].

\subsubsection{Garp}

A arquitetura Garp [Callahan et al. 2000] pretende amenizar o acesso limitado à memória encontrado em arquiteturas como a Chimaera, assegurando assim maior exploração do potencial da lógica reconfigurável. Este sistema, ao contrário dos descritos anteriormente, permite que a lógica configurável tenha acesso direto à memória, ao invés de transferir todos os dados 
necessários via registradores do processador hospedeiro. A integração direta da memória e da lógica reconfigurável aumenta a quantidade de dados disponíveis para os recursos configuráveis, possibilitando deste modo explorar melhor o paralelismo e aumentar o desempenho.

Nesta arquitetura, cuja organização básica é ilustrada na Figura 5.9, há a integração de um processador MIPS e um coprocessador programável. Este coprocessador é otimizado para a aceleração de ciclos em aplicações de software de uso geral, recorrendo à regra 90/10, que postula que $90 \%$ do tempo de execução de um programa é despendido em 10\% de seu código, sendo necessário, portanto, acelerar esta pequena porção do código com ajuda de FPGA.

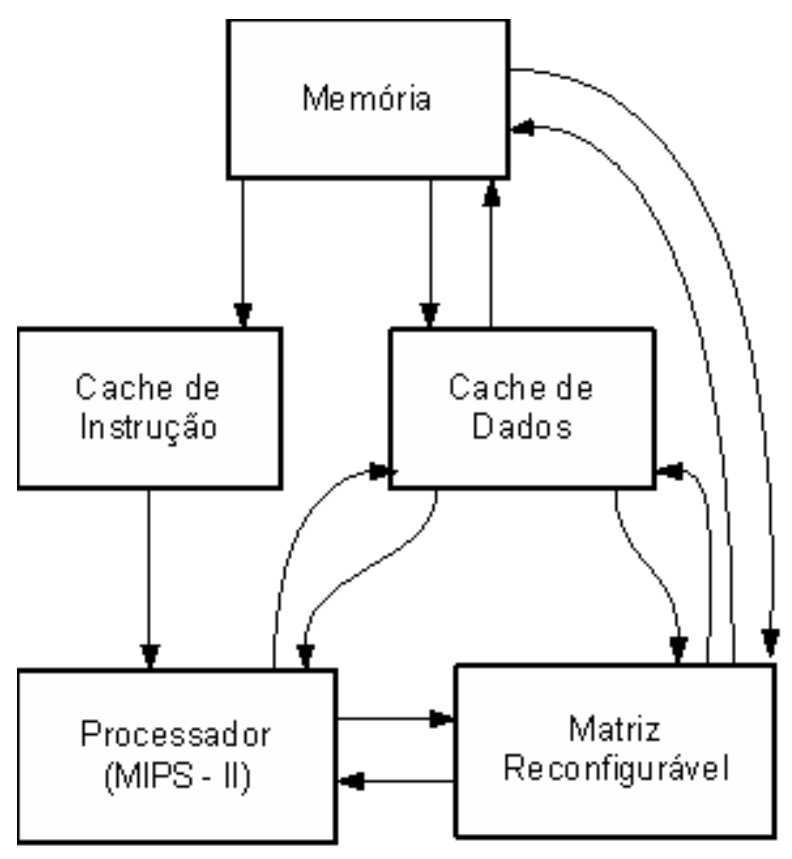

Figura 5.9 - Organização básica da arquitetura Garp em diagrama de blocos [Costa 2007].

Para que o tempo de reconfiguração fosse reduzido, o coprocessador inclui uma cache que mantém configurações recentemente removidas. Depois de configurado, o Garp pode executar de modo eficiente, podendo ser interrompido a qualquer momento pelo processador.

O compilador desenvolvido para o Garp adapta muitas técnicas propostas previamente para a exploração do paralelismo ao nível de instruções em processadores VLIW (Very Long Instruction Word). O fluxo de compilação consiste na identificação de porções do código C que 
podem ser executadas pelo coprocessador reconfigurável, síntese do código de interface entre hardware/software, e das configurações da lógica reconfigurável.

Arquiteturas como a DISC [Wirthlin \& Hutchings 1995], MATRIX [Mirsky \& DeHon 1996], RaPiD [Ebeling et al. 1996], PipeRench [Goldstein et al. 2000], ADRES [Mei et al. 2003], Tartan [Mishra \& Goldstein 2007], WASMII [Ling \& Amano 1993], MorphoSys [Singh et al. 2000], HSRA [Tsu et al. 1999], ARRIVE [Köhler et al. 2008] entre outras, apesar de não ter sido descritas aqui, contribuíram com a definição de muitos conceitos sobre computação reconfigurável, demonstrando seus benefícios de capacidade de processamento e desempenho.

\subsection{Reconfiguração Parcial Dinâmica nas Arquiteturas Virtex da Xilinx}

A Xilinx é uma empresa pioneira na fabricação de FPGAs com suporte à reconfiguração parcial. Com a família Virtex, é possível desempenhar essa reconfiguração de maneira dinâmica, em tempo de execução. Nesta seção, esta família de FPGAs, que foi escolhida para este trabalho de pesquisa, será descrita, destacando-se as maneiras pelas quais a reconfiguração parcial é feita.

Como mostra a Figura 5.10, os dispositivos da família Virtex-II são compostos por blocos lógicos configuráveis (CLBs), com blocos de entrada e saída (IOBs) formando o perímetro. Além disso, há módulos de memória SelectRAM podendo ser lidos ou escritos sem a necessidade de parada do dispositivo [Xilinx 2007a]. A menor unidade de memória de configuração que pode ser lida ou escrita é um quadro (frame), que ocupa toda a altura do dispositivo, incluindo IOBs.

Além dos blocos básicos, há também multiplicadores dedicados para dados de até 18 bits, sendo cada um desses blocos associado a um bloco de memória SelectRAM, tornando operações para DSP mais eficientes, por exemplo. Os blocos DCM (Digital Clock Manager), juntamente com o clock, fornecem uma solução completa para qualquer sistema síncrono, provendo gerenciamento de atrasos de clocks distribuídos [Xilinx 2007a]. 


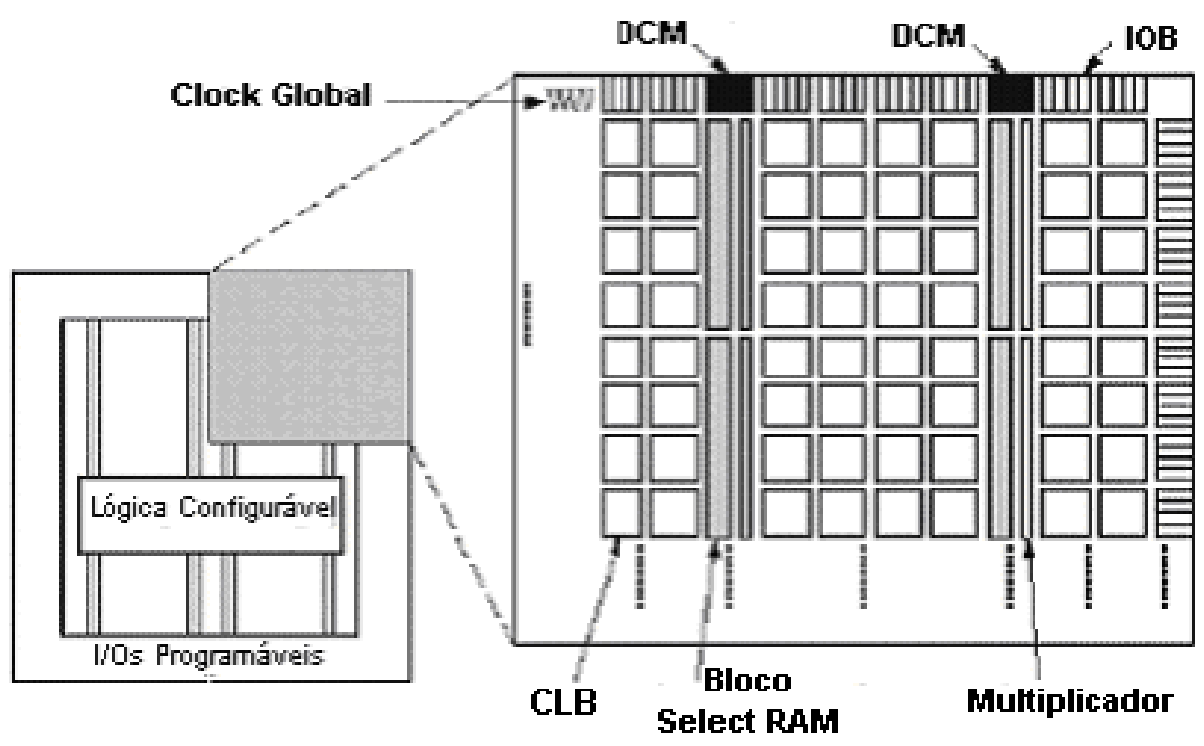

Figura 5.10 - Arquitetura dos FPGAs Virtex-II [Adaptado de Xilinx 2007a].

Os CLBs da arquitetura Virtex-II consistem de quatro slices, cada um deles contendo duas células lógicas, que são, por sua vez, formadas de um gerador de função de quatro entradas, que é uma LUT de quatro entradas, lógica carry ("vai-um" propagado), e elemento de armazenamento. Os CLBs das Virtex-II também contém portas lógicas aritméticas e multiplexadores, usados para combinar geradores de função.

Para que seja feita a interconexão entre os recursos lógicos, é utilizada uma técnica de implementação da estrutura de roteamento, chamada Active Interconnect Technology. O GRM (General Routing Matrix) é um array de comutadores de roteamento. Cada elemento programável é ligado a uma matriz de comutadores, permitindo múltiplas conexões ao GRM. As interconexões programáveis globais são hierárquicas e projetadas para suportar altas freqüências. Todos os elementos programáveis, incluindo os recursos de roteamento, são controlados por valores armazenados em células de memória estática (SRAM). Estes valores são carregados nas células de memória durante a configuração e podem ser recarregados para mudar as funções dos elementos programáveis [Xilinx 2007a]. 
As últimas gerações dos FPGAs Virtex, as famílias Virtex-4 [Xilinx 2007b] e Virtex-5, têm diferenças significativas em sua arquitetura, se comparadas com as Virtex-II. Nos FPGAs Virtex-4, como mostra a Figura 5.11, a arquitetura de configuração ainda é baseada em quadros (frames), mas cada quadro ocupa 16 linhas de blocos CLB ao invés de toda a altura do dispositivo, sendo teoricamente mais flexíveis para RTR. As regiões de distribuição de clocks nas Virtex-4 estão alinhadas em blocos de 16 linhas de CLBs, ao contrário da Virtex-II, nas quais as regiões de clock são definidas como quadrantes. Outra diferença encontrada nas Virtex-4 é a disposição dos blocos IOB, que estão arranjados em colunas, ao invés de estarem distribuídos no perímetro do FPGA.

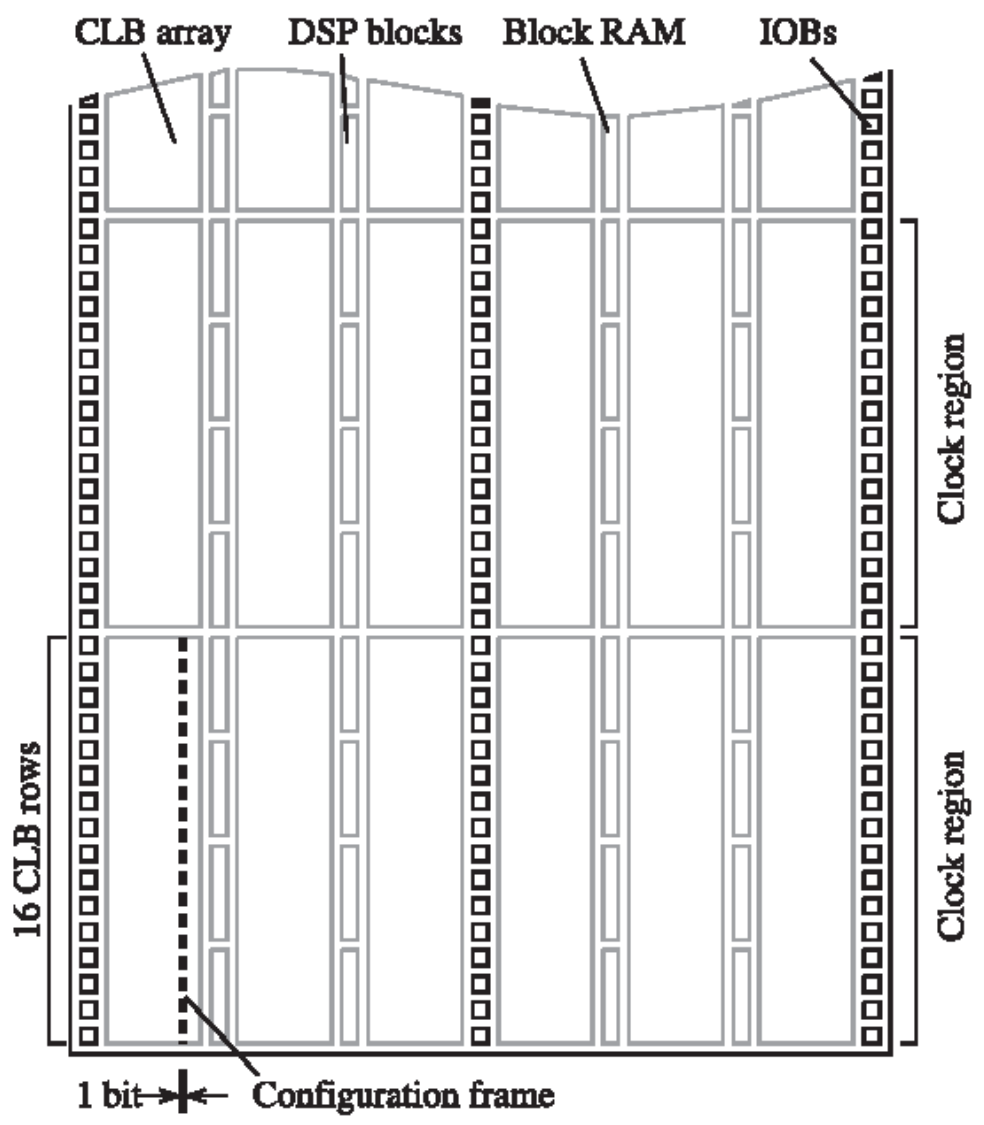

Figura 5.11 - Arquitetura dos FPGAs Virtex-4 [Xilinx 2007b].

Os FPGAs Virtex-5 [Xilinx 2007c], se comparado com a família Virtex-4, têm maior capacidade e desempenho, chegando a ter pouco mais de 330.000 células lógicas. Utilizam 
tecnologia de processo de $65 \mathrm{~nm}$, enquanto os anteriores usam 90nm, o que permite maior densidade e desempenho. Além disso, possuem blocos de memória RAM e registradores SRL (Shift Register LUT) maiores, permitindo o uso de mais memória e pipelines com mais estágios. Utilizam LUTs com seis entradas independentes, possibilitando a utilização de menos níveis lógicos, que aumenta a densidade e a velocidade. A técnica de roteamento utilizada pelas Virtex5 é mais rápida e previsível, feita de modo diagonal, ao invés de ser segmentada como nas famílias anteriores.

Nos FPGAs da Xilinx, a reconfiguração parcial pode ser feita de duas maneiras diferentes [Xilinx 2005] [Kao 2005]. A primeira delas, baseada em módulos, é utilizada para a reconfiguração de lógica em blocos complexos. Já a segunda, baseada em diferenças, é utilizada para alterar pequenas partes do FPGA. Estes métodos serão estudados com mais detalhes a seguir.

\subsubsection{Reconfiguração Parcial Baseada em Módulos}

Considerando seu arranjo físico, tanto da lógica quanto dos recursos de roteamento, as arquiteturas FPGA impõem restrições às quais os projetos parcialmente reconfiguráveis devem aderir. A reconfiguração parcial baseada em módulos se atém à metodologia de projeto modular (Modular Design), da Xilinx, que permite a partição de um projeto em subsistemas, facilitando o desenvolvimento dos módulos em paralelo (engenharia concorrente) e o reuso dos módulos, o que propicia o aumento da produtividade e qualidade do projeto [Xilinx 2005].

Utilizando a metodologia de projeto modular, a reconfiguração parcial baseada em módulos é usada para alterar blocos grandes de lógica, denominados módulos de reconfiguração parcial (Partial Reconfiguration Modules - PRM), ilustrados na Figura 5.12. A definição desses módulos deve obedecer a restrições do desenvolvimento modular, além de restrições específicas dos projetos com reconfiguração parcial, descritas a seguir [Mermoud 2004] [Xilinx 2005].

- Restrições de Área - uma vez definidas, as fronteiras entre os módulos não podem ser modificadas. Nos FPGAs da família Virtex-II, por exemplo, os módulos devem ocupar toda a altura do dispositivo. Toda lógica encontrada dentro de um módulo, 
incluindo CLBs, IOBs, blocos de RAM, e recursos de roteamento, é reservada somente àquele módulo.

- Restrições de I/O - IOBs encontrados ao longo da fronteira de um módulo são de uso exclusivo daquele módulo. Esta regra não se aplica aos clocks, que possuem quadros de bitstream separados.

- Restrições de Comunicação - Para que um módulo possa se comunicar com outros módulos, fixos ou reconfiguráveis, é necessária a utilização de macros de barramentos, ou bus macros.

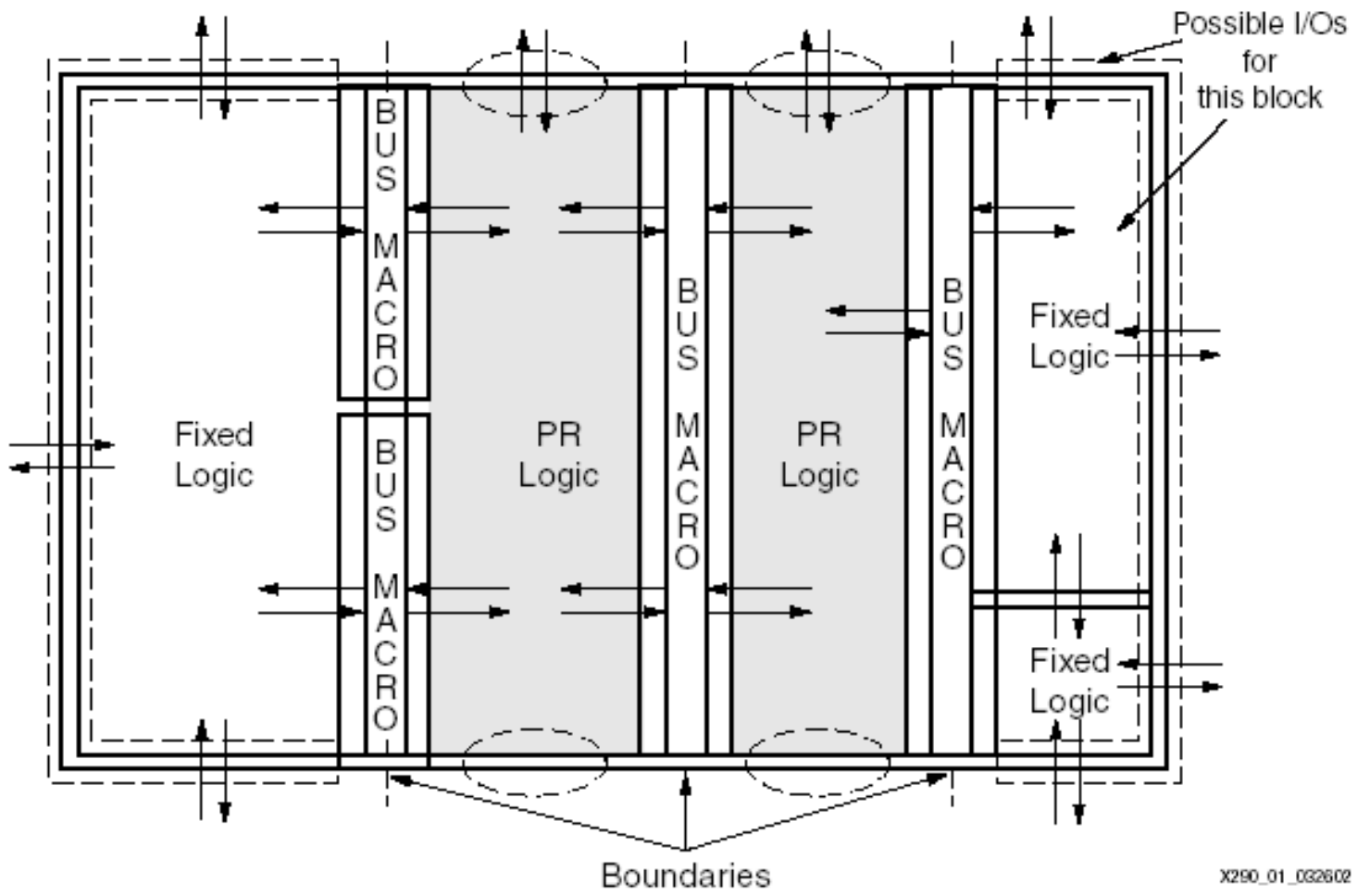

Figura 5.12 - Projeto com módulos reconfiguráveis [Xilinx 2005].

Para que a implementação de um projeto com reconfiguração parcial seja feita com sucesso, como já foi dito, é necessário seguir uma metodologia estrita de projeto. Algumas normas que devem ser observadas são as seguintes: 
- É necessário inserir bus macros entre módulos PRM e o resto do projeto, composto por lógica estática.

- Uma netlist parcialmente reconfigurável deve ser gerada seguindo diretrizes de síntese específicas do fluxo de projeto parcialmente reconfigurável.

- Fazer a representação esquemática da disposição inicial dos PRM (floorplan) e agrupar logicamente todos os módulos estáticos.

- Bus macros devem ser inseridos entre os PRMs e a lógica estática.

- As regras específicas da reconfiguração parcial devem ser seguidas e o processo de implementação da reconfiguração parcial deve ser executado, como será mostrado a seguir.

O software PlanAhead, da Xilinx, provê uma plataforma única para o gerenciamento destas normas [Dorairaj et al. 2005], sendo o primeiro ambiente gráfico que dá suporte ao processo de reconfiguração parcial. Para que um projeto com reconfiguração parcial seja implementado com o PlanAhead, é necessário seguir uma lista de passos, descritos a seguir.

- Importar netlist - o software utiliza qualquer netlist sintetizada, como XST ou Synplify, que é uma netlist gerada especificamente para uma determinada plataforma alvo.

- Desenvolver o floorplan para a reconfiguração parcial - é necessário, para isso, estabelecer um PRM, apontando uma área no FPGA para ele. Após isso, estabelece-se a lógica estática e inserem-se os bus macros.

- Verificação das regras de projeto - devido à complexidade do processo, é comum introduzir erros no RTL original e durante o processo de floorplanning. Por isso, o PlanAhead verifica se há erros no projeto.

- Exportar netlist - após o processo de floorplanning e de verificação, uma netlist específica para a reconfiguração parcial é exportada. 
- Gerenciamento do processo de implementação - neste passo, serão produzidos um bitstream para o projeto em sua totalidade, além de um bitstream para cada um dos PRMs.

- Estimativa do tamanho do bitstream - essa informação pode ser utilizada para a estimação do tamanho da memória de configuração, como uma flash externa. $\mathrm{O}$ tamanho do bitstream pode também ser usado para o cálculo do tempo necessário para a troca de módulos, levando em consideração o desempenho da interface de memória na qual está o bitstream.

A técnica de reconfiguração parcial baseada em módulos possui algumas limitações, ao menos nos dispositivos Virtex-II [Xilinx 2007a]. O fluxo de projeto que cria os bitstreams para a porção estática além dos módulos reconfiguráveis, restringe a posição dos módulos ao nível máximo na hierarquia lógica do projeto. Para dispositivos grandes, o uso de toda a altura pode tornar o uso de recursos ineficiente. Finalmente, enquanto ocorre reconfiguração em um módulo, as partes do sistema de ambos os lados desse módulo ficam isoladas entre si, devido ao reroteamento dos caminhos de cada sinal. Entretanto, o tamanho reduzido dos quadros nos dispositivos Virtex-4 e Virtex-5 deve remediar esses problemas, uma vez que os módulos podem ter 16 CLBs de altura (ou um múltiplo de 16), ao invés de toda a altura do dispositivo. Isso significa que os recursos podem ser alocados de modo mais eficiente, e as áreas estáticas não ficam necessariamente isoladas uma das outras [Sedcole et al. 2006].

\subsubsection{Reconfiguração Parcial Baseada em Diferenças}

Outra maneira de se fazer a reconfiguração parcial nos dispositivos Virtex é utilizar o modelo que se baseia em diferenças [Xilinx 2007e]. Este método é utilizado para alteração de pequenas partes do FPGA, como padrões de E/S, equações de uma LUT, e o conteúdo de um bloco RAM, por exemplo, não sendo recomendado para alterações significativas do FPGA. Apesar de ser possível mudar informações de roteamento, não é recomendável por causa da possibilidade de haver conflitos internos durante a reconfiguração. Se mudanças de roteamento forem necessárias, esta metodologia não é a recomendada. 
O projetista pode fazer as pequenas alterações no projeto utilizando a ferramenta FPGA_Editor. Após a alteração do projeto, o programa BitGen é utilizado para gerar um bitstream que programa apenas a diferença entre as duas versões do projeto. A mudança da configuração de um módulo de uma implementação para outra é muito rápida, porque o bitstream das diferenças pode ser bem menor que um bitstream do dispositivo inteiro.

Atualmente, a documentação da Xilinx a respeito deste tipo de reconfiguração apenas dá suporte à mudança de padrões de $\mathrm{I} / \mathrm{O}$, conteúdo de blocos RAM, e programação das LUTs [Xilinx 2007e]. No FPGA_Editor, o menor elemento lógico que pode ser escolhido é o slice. A partir daí, pode-se ver, por exemplo, equações de uma LUT. A Figura 5.13 ilustra o esquemático de um slice no ambiente FPGA_Editor. Em caso de alterações do conteúdo de blocos de memória RAM, a ferramenta Data2MEM pode ser utilizada no lugar da FPGA_Editor. Caso esta alteração, assim como a de padrões de I/O, seja feita no FPGA_Editor, as metodologias são semelhantes à utilizada para alterações de equações em LUTs, como descrito em [Xilinx 2007e].

No que se refere à programação do FPGA, para a realização da reconfiguração parcial pode-se utilizar as interfaces de configuração SelectMAP e JTAG, ou a ICAP para a autoreconfiguração. O aplicativo de configuração da Xilinx, iMPACT, pode ser utilizado para testes de reconfiguração. Alternativamente, os projetistas podem criar funções em nível de sistema para o controle da reconfiguração. Uma vez que os dispositivos não são capazes de distinguir entre bitstreams parciais e bitstreams completos, o projetista precisa ser cauteloso ao carregar os bitstreams nos dispositivos para não comprometer o funcionamento do mesmo [Xilinx 2007e]. 


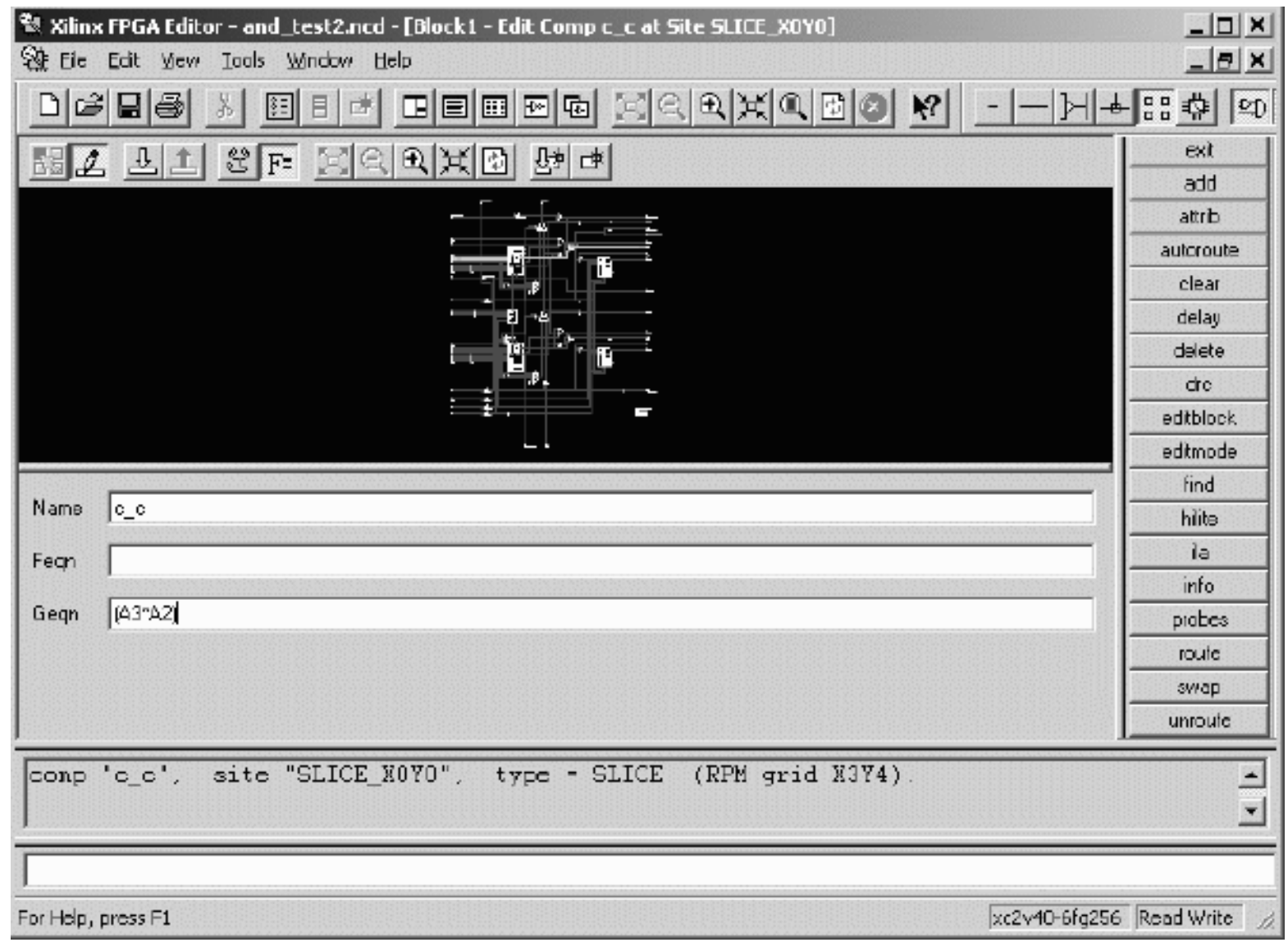

Figura 5.13 - FPGA Editor mostrando o esquemático de um slice [Xilinx 2007e]. 


\section{6}

\section{Modelo de Reconfiguração Parcial para o ChipCflow}

Conforme o modelo de instâncias apresentado no Capítulo 3, a proposta inicial do projeto ChipCflow contemplava um modelo completamente flexível de geração e execução de instâncias de operadores ou subgrafos em áreas parciais nos FPGAs Virtex. Isto é, inicialmente, um circuito de matching detectaria a existência de dados cujos parceiros ainda não estão disponíveis. Caso isso acontecesse, uma nova instância do operador seria gerada dinamicamente no circuito. Da mesma forma, tão logo que uma instância fosse executada por ter presentes todos os tokens de cada porta de entrada, a mesma seria retirada do circuito, o que geraria, assim, uma estrutura totalmente flexível à medida que as instâncias fossem geradas e executadas. Com o aprofundamento do estudo do processo de reconfiguração parcial dinâmica, chegou-se à conclusão que tal modelo não era viável utilizando-se as técnicas atuais. Esse estudo foi feito tendo como fonte, em sua maioria, documentações diversas obtidas na página Partial Reconfiguration Early Access Tools Lounge, no website da Xilinx, que disponibiliza, mediante registro, acesso antecipado a aplicativos e documentos referentes à reconfiguração parcial, antes seu lançamento oficial [Xilinx URL b]. Pode-se citar como documentação principal de referência para o estudo e desenvolvimento do trabalho descrito neste e nos demais Capítulos, Xilinx [2006a] [2008a] [2007f] [2007g] [2008b] [2008c] [2007h], [Mermoud 2004], e [Lysaght et al. 2006]. 
Exceto em alguns raros exemplos onde há, por exemplo, processamento linear com uma única fonte de tokens, em principio qualquer operador de um grafo ChipCflow, com mais de uma porta de entrada, tem o potencial de ser replicado em áreas parcialmente reconfiguráveis. Por exemplo, um "grafo" a fluxo de dados extremamente simples, composto apenas por um operador de adição, pode ter esse operador replicado, dependendo da freqüência de alimentação dos tokens de entrada. No modelo atual de reconfiguração baseada em módulos da Xilinx, a única aplicável ao projeto, é necessário um projeto-base em lógica estática, no qual os bus macros são inseridos para tornar possível a transmissão de dados entre a lógica estática e a reconfigurável. A definição das áreas de reconfiguração e das posições dos bus macros deve ser feita manualmente, por meio da utilização de softwares como o Xilinx PlanAhead. Por isso, é impossível implementar um mecanismo completamente flexível, mesmo porque é necessário, como vimos, um projeto-base estático, não sendo possível ligar regiões reconfiguráveis sem tê-lo como intermediário. Segundo a documentação da Xilinx, todos os módulos parcialmente reconfiguráveis possíveis precisam ser implementados e ter seus bitstreams gerados antes da programação do FPGA, e a lógica estática precisa ser implementada e gerada antes dos módulos reconfiguráveis. Por isso, os operadores ou subgrafos a serem replicados precisam ser definidos com antecedência.

Considerando os motivos apresentados, foi decidido pela utilização da versão estática do grafo a fluxo de dados como o projeto-base do modelo, acrescentado de módulos parcialmente reconfiguráveis auxiliares para tornar possível a reconfiguração parcial e, conseqüentemente, o processamento a fluxo de dados dinâmico, uma vez que nesses módulos estarão os operadores replicados. Como não é o propósito deste projeto analisar a partição do grafo a fluxo de dados, apenas operadores estarão replicados, e não subgrafos. Assim, como mostra a Figura 6.1, cada operador que possui mais de uma porta de entrada, tendo, portanto, potencial de ser replicado, está ligado a uma região reconfigurável. No caso, o algoritmo é basicamente o exemplo dos comandos IF-THEN-ELSE, ilustrado na Figura 3.6b, no Capítulo 3, sendo ele o seguinte:

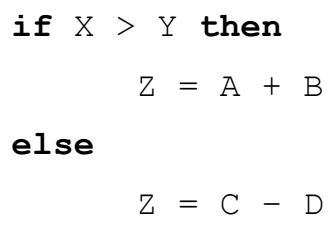


Como mostra a Figura 6.1, há duas regiões parcialmente reconfiguráveis, designadas como PRR1 e PRR2, às quais estão associados os operadores do grafo. Os operadores de decisão e o de adição estão associados à primeira, já o de junção determinística e o de subtração estão associados à segunda. Portanto, a cada região parcialmente reconfigurável (Partially Reconfigurable Region - PRR) estão associados dois operadores, não sendo possível replicar os dois simultaneamente em uma mesma região. Essa restrição é necessária porque o número de regiões reconfiguráveis em um FPGA é limitado, e não é possível atribuir uma região qualquer do grafo a fluxo de dados ou um operador, a ser replicado, a uma região qualquer que esteja livre, de forma aleatória, pelos motivos apresentados acima (impossibilidade de flexibilidade total).

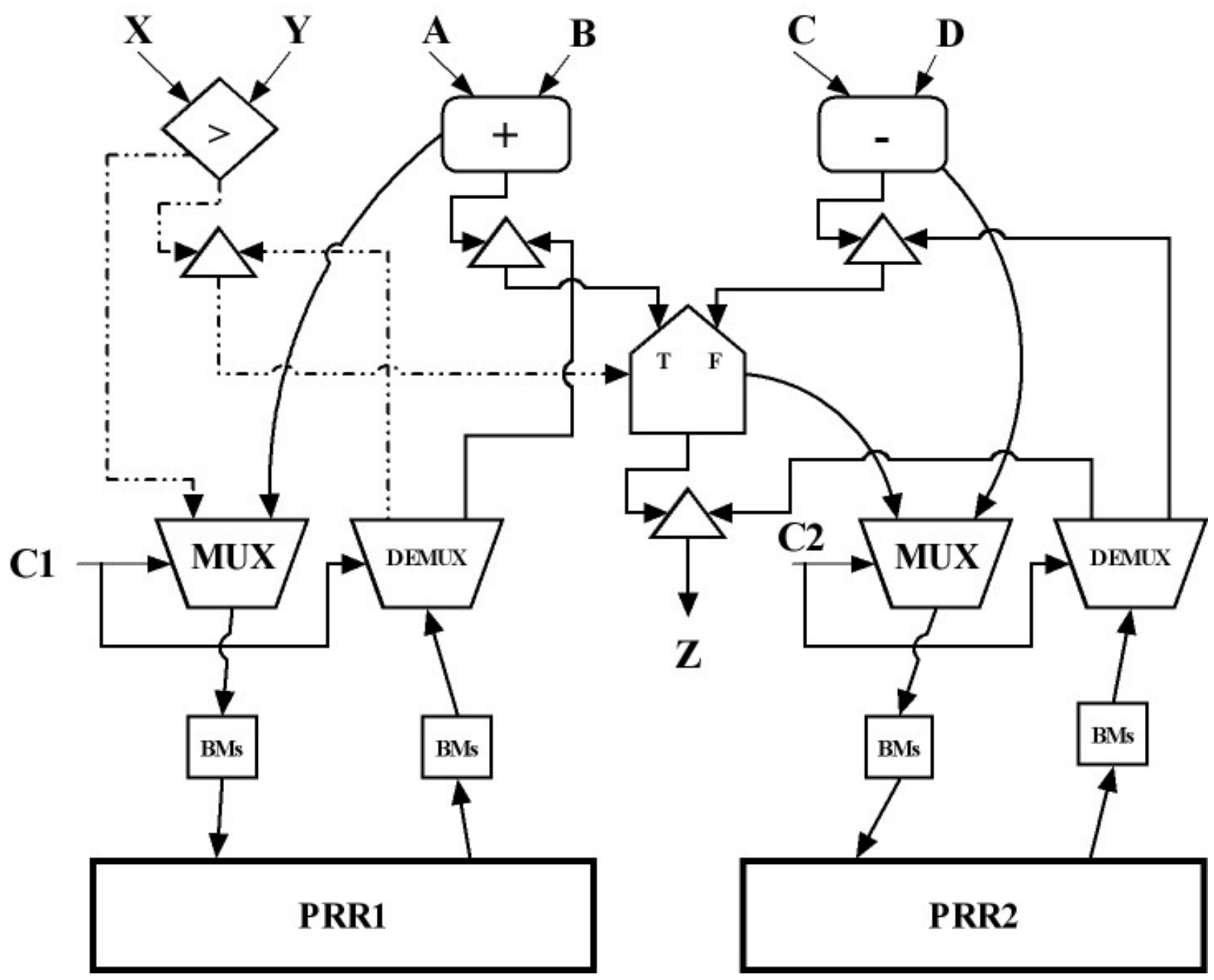

Figura 6.1 - Exemplo que ilustra o modelo de reconfiguração parcial dinâmica para o projeto. 
Quando ocorre algum conflito, uma parte do grafo a fluxo de dados se torna estática, porque elementos replicados de duas regiões do grafo (no caso da figura, dois operadores) não podem ocupar uma mesma PRR ao mesmo tempo. Por exemplo, se a região PRR1 já estiver sendo utilizada pelo operador de decisão, o operador de adição necessariamente se comportará de modo estático, pois não será possível replicá-lo.

No modelo apresentado pela Figura 6.1, quando chega um token a um operador, como o de decisão, ele é armazenado em um registrador neste operador. Uma vez que seu token parceiro chega ao mesmo operador, ocorre o disparo e o token resultante é enviado a um operador de junção não-determinística, apresentado no Capítulo 2, responsável pelo envio do token ao operador subseqüente. Caso chegue um token em uma porta de entrada na qual já exista, em seu respectivo registrador, um token esperando por seu parceiro, é requisitada a reconfiguração parcial da PRR correspondente ao operador. Caso ela estiver sendo utilizada por outro operador, o operador que fez a requisição simplesmente se comporta como um operador a fluxo de dados estático, recusando o token que chegou até a chegada do parceiro do token armazenado. Quando um operador "recusa" um token, não enviando um sinal de confirmação de recebimento, esse token continuamente será reenviado até que ele seja aceito. Se, porém, a reconfiguração tenha sido feita com sucesso, o token é enviado a um operador replicado no módulo reconfigurável, que por sua vez aguardará por seus parceiros. Para que seja feito esse envio, é utilizado um multiplexador (MUX), que combina, em um único fluxo de dados, um conjunto de sinais vindo de todos os operadores que estão alocados a uma determinada PRR. Os demultiplexadores (DEMUX), por sua vez, são utilizados para o envio de tokens originários dos operadores replicados nas PRRs ao operador de junção determinística correspondente ao operador do qual derivam os replicados. Esses multiplexadores e demultiplexadores são necessários porque tokens podem originar ou serem direcionados a todos os operadores que utilizam uma determinada PRR, sendo necessário, portanto, um mecanismo para o compartilhamento dos bus macros da PRR. Sinais de controle, no caso $\mathbf{C 1}$ e $\mathbf{C 2}$, são utilizados para determinar para qual operador uma determinada PRR está sendo ocupada. Entre os módulos parcialmente reconfiguráveis e os MUXes e DEMUXes há bus macros, denotados por BMs, que, como já visto, são necessários ao processo de reconfiguração. O gerenciamento do envio dos tokens por operadores de junção não 
determinística é necessário porque, como ilustra a figura, há duas fontes de chegada de tokens: o próprio operador e o demultiplexador ligado à PRR.

Como ilustra a Figura 6.2, são necessários controladores de entrada e saída, representados como CE e CS, nos módulos parcialmente reconfiguráveis (Partial Reconfiguration Module PRM), para que a atribuição dos tokens às portas de entrada dos operadores replicados seja feita de maneira correta. No controlador de entrada deverá ser implementado, como trabalho futuro, a estrutura de matching, tendo como base a proposta por Silva [2006], fundamental para o funcionamento do modelo em sua plenitude. Este trabalho não tem como foco a implementação do protocolo de comunicação necessário para a identificação dos tokens (isto é, os próprios tagged-tokens) entre os diversos módulos de um grafo dinâmico, cujo estudo está sendo feito por outro mestrando pertencente ao grupo de pesquisa do ChipCflow [Souza 2008]. Note que o PRM ilustrado na figura se destina à replicação do operador de adição, e que, nesse exemplo simplificado, há apenas três operadores replicados.

Os sinais de controle C1 e C2, assim como os responsáveis pelas solicitações de reconfiguração parcial, deverão ser enviados ou recebidos por um Gerenciador de Reconfigurações (GR), como apresentado por Souza [2008]. A principal função do GR é administrar quais PRMs estarão alocadas aos PRRs, controlando assim a reconfiguração parcial. Essa lógica de controle para a reconfiguração parcial pode residir em um dispositivo externo, como um processador, ou dentro do próprio FPGA a ser reconfigurado, sendo esse um controlador de reconfiguração parcial interno. O controlador carrega pela interface ICAP bitstreams parciais que podem residir, por exemplo, em um dispositivo Xilinx System ACE CompactFlash. Como todo o resto da lógica estática que consiste o projeto base, os circuitos do controlador operam sem interrupção durante o processo de reconfiguração parcial. Na plataforma Virtex, um controlador interno de reconfiguração parcial pode ser consistido de uma máquina de estados padrão ou um processador embarcado como o Microblaze ou o Power PC 405. Além disso, os bistreams parciais podem ser carregados no FPGA pela porta JTAG, utilizando-se a ferramenta iMPACT, do Xilinx ISE. 


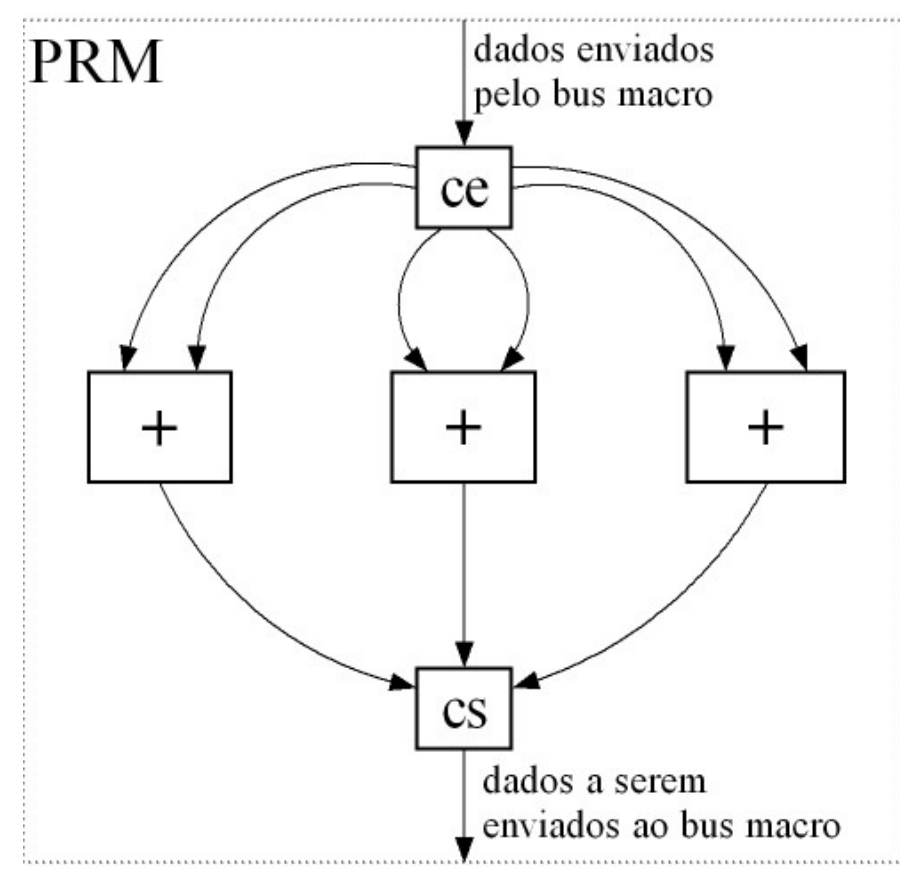

Figura 6.2 - Modelo de um módulo parcialmente reconfigurável (PRM).

O modelo apresentado pode ser considerado uma arquitetura a fluxo de dados híbrida direcionada à execução dinâmica nos casos ideais, e estática quando o compilador não tem sucesso em garantir a distribuição das PRRs às áreas do grafo que realmente as utilizarão de maneira efetiva. A execução é dinâmica quando há cópia de lógica na forma de operadores (ou subgrafos) replicados, o que garante maior nível de concorrência, sendo possível enviar continuamente tokens de uma determinada porta de entrada a operadores replicados sem a necessidade de espera pelos seus respectivos tokens parceiros. A máquina a fluxo de dados, entretanto, se comporta de maneira estática quando não houver possibilidade de replicação dos operadores. 


\section{Implementação dos Operadores do ChipCflow}

Utilizou-se as ferramentas EDA ISE 8.2i Foundation e 9.1i Foundation, da Xilinx, para a implementação dos operadores a fluxo de dados e demais módulos, além dos próprios algoritmos representados por grafos a fluxo de dados do modelo apresentado no Capítulo 6. A simulação da lógica foi feita utilizando-se o software Xilinx ISE Simulator, já incorporado ao ISE.

Como metodologia para o desenvolvimento do projeto, inicialmente implementou-se os operadores para o modelo a fluxo de dados estático, sendo também implementados, a partir desses operadores, vários grafos estáticos, para fins de teste. Também foram implementados diversos módulos auxiliares, utilizados para que a execução dos grafos ocorresse da maneira correta. Em seguida, adaptou-se os operadores para o modelo dinâmico proposto, implementouse os módulos auxiliares descritos no Capítulo 6, como os MUXes, DEMUXes, e os PRMs. Finalmente foi implementado, como prova de conceito, um algoritmo na forma de grafo a fluxo de dados que utiliza o modelo proposto, sendo seu desenvolvimento descrito com mais detalhes no Capítulo 8.

\subsection{Modelo a Fluxo de Dados Estático}

O entendimento do modelo a fluxo de dados estático é fundamental para a implementação do modelo proposto, uma vez que o projeto-base será um grafo a fluxo de dados estático, 
acrescido dos módulos necessários para a replicação dos operadores, que caracteriza o modelo a fluxo de dados dinâmico. O processo de desenvolvimento dos grafos em hardware foi dividido em duas partes. Inicialmente, foram implementados os módulos de baixo nível, isto é, os operadores básicos do modelo a fluxo de dados estático, descritos no Capítulo 2. Nesse caso, foi utilizada a linguagem VHDL, porque ela oferece maiores níveis de abstração, se comparada com blocos esquemáticos [Pedroni 2004]. Já os módulos de alto nível - os grafos a fluxo de dados foram criados utilizando-se blocos esquemáticos, cuja principal característica é a representação visual do circuito, o que é desejável, considerando que os algoritmos a fluxo de dados são propostos na forma de grafos.

\subsubsection{Elaboração dos Operadores Estáticos}

Foi definido, inicialmente, que os dados utilizados nas operações teriam 16 bits. Dessa maneira, todos os operadores foram criados utilizando barramentos dessa largura. Desde cedo, percebeu-se que seria muito difícil implementar os operadores por meio de blocos esquemáticos. Assim, foi utilizada a linguagem de descrição de hardware VHDL.

Notou-se que, no caso dos operadores, seria melhor desenvolve-los como simples máquinas de estados. Desta maneira, foi especificado que o operador poderia estar em três estados: aguarda tokens, executa, e envia tokens. Quando inicializado, o operador está no estado aguarda tokens, que é responsável pela recepção dos tokens e armazená-los em registradores. Após receber os seus tokens de entrada, o operador fica habilitado, havendo assim a mudança para o estado executa, onde o operador é "disparado". Após o disparo, o operador assume o estado envia tokens. Uma vez recebidos os sinais acknowledge, indicando que os dados foram enviados corretamente ao operador subseqüente, o operador que enviou o token volta a seu estado inicial (aguarda tokens). A Figura 7.1 ilustra o diagrama de máquina de estados como concebido inicialmente. Na notação utilizada, o círculo preenchido com cor preta indica o estado inicial.

Mais tarde, percebeu-se que o estado executa poderia ser eliminado para aumento de desempenho, pois não seria perdido um ciclo de clock com a execução, que foi incorporada a um 
dos outros dois estados. Por exemplo, no caso do operador de adição, tão logo que o operador recebe seus dois tokens de entrada, os dados são somados e guardados em um registrador, daí o estado muda para envia token, no qual um token contendo o valor do registrador é enviado ao nó subseqüente do grafo.

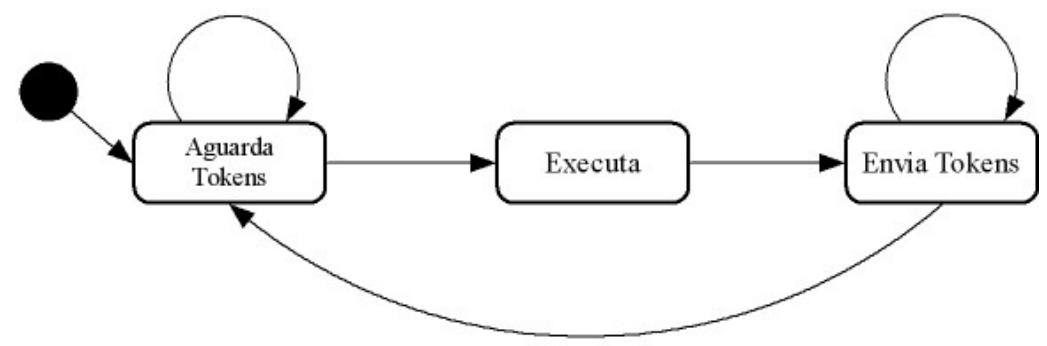

Figura 7.1 - Diagrama de máquina de estados, em notação SysML, dos operadores estáticos.

Quando um operador está no estado envia tokens, em cada ciclo de clock ele envia seus tokens de saída para os operadores aos quais seus arcos de saída estão ligados. É necessário, portanto, que ele saiba quando parar de enviar e voltar ao estado inicial. Isso é feito utilizando-se sinais de reconhecimento (acknowledge ou ack). Similarmente, sinais de strobe são utilizados para indicar a um operador que outro operador ao qual ele está ligado está enviando um token. Esses sinais têm um bit de largura, podendo assumir valor verdadeiro (um) ou falso (zero). Na Figura 7.2 está ilustrada a representação esquemática do bloco responsável pela operação de adição no modelo estático (OPADD). O operador OPADD, como mostra a figura, possui dez sinais de E/S: a, b, astr, bstr, aack, back, z, zstr, zack, e clkin. Os sinais a e b são os dados de entrada; astr e bstr são seus respectivos sinais de strobe que indicam que há um novo dado chegando às entradas a e b, respectivamente. As saídas aack e back são os sinais de acknowledge para as entradas a e b. A saída $\mathbf{z}$ contém a soma dos dados de entrada $\mathbf{a}$ e $\mathbf{b}$, sendo zstr e zack respectivamente seus sinais de strobe e acknowledge. Finalmente, clkin é o sinal de clock. A Figura 7.3 mostra o diagrama de estados e a parte arquitetural do código VHDL do operador OPADD. 


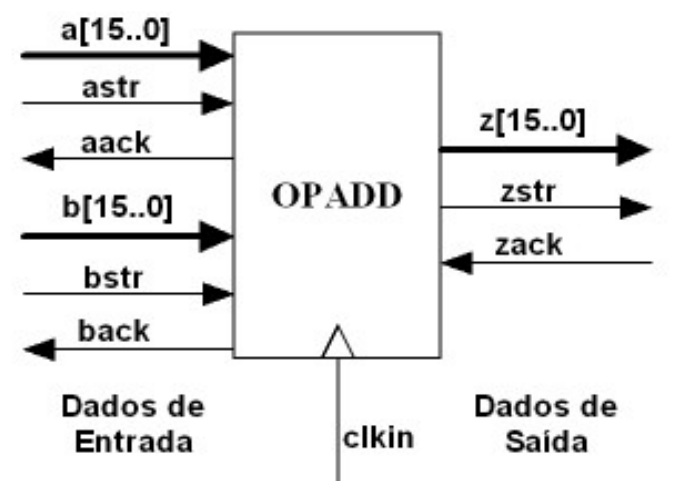

Figura 7.2 - Bloco esquemático OPADD - operador estático de adição.
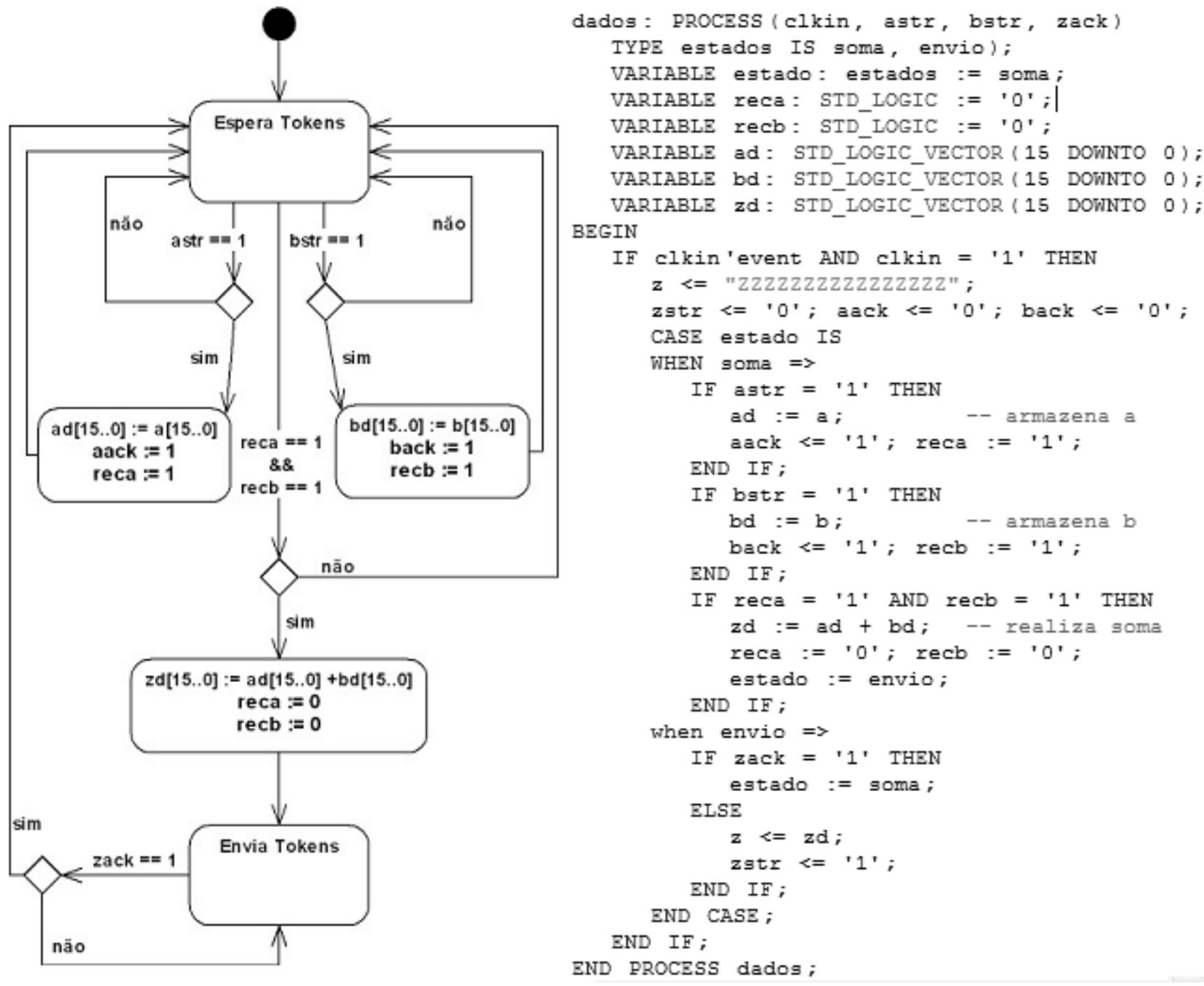

Figura 7.3 - Operador OPADD: Diagrama de estados e parte arquitetural do código VHDL. 
A seguir, será descrito de maneira breve o funcionamento do operador. Inicialmente, o sistema se encontra no estado espera tokens. Caso um token chegue a sua porta de entrada a ou b, por meio da checagem dos valores dos sinais de strobe (astr e bstr, respectivamente), esse valor é armazenado em registradores, no caso, ad ou bd. Além disso, são utilizados registradores auxiliares que assumem o valor "um" quando um token é recebido na porta de entrada, sendo eles reca e recb. Quando ambos registradores assumem valor "um", a soma é realizada e armazenada no registrador zd. Além disso, reca e recb assumem novamente o valor "zero", e o estado muda para envia tokens. O sistema permanecerá nesse estado até que o operador receba, na entrada zack, a indicação de que o token foi enviado com sucesso ao operador subseqüente.

Utilizando a mesma metodologia descrita até agora, foram implementados todos os operadores básicos do modelo a fluxo de dados estático, isto é: branch, value deterministic merge, nondeterministic merge e copy. No caso dos operadores (operators), para cada tipo de operação básica foi desenvolvido um operador diferente. Quatro operadores, portanto, foram criados, incluindo o OPADD. Quanto ao decider, foram criadas cinco variações desse operador, para cada tipo de comparação, isto é: maior, maior ou igual, menor, menor ou igual, e igual. Por razões de brevidade, as implementações desses operadores não serão descritas em detalhes, uma vez que elas são semelhantes à do OPADD.

Após ser sintetizado, no que se refere à utilização lógica, constatou-se que o operador OPADD utilizou 25 slices, 39 slice flip-flops, 28 LUTs de entrada, e 8 TBUFs.

\subsubsection{Implementação de Grafos Estáticos}

Após a implementação e teste dos operadores básicos do modelo a fluxo de dados estático, foram gerados, para a criação dos grafos, blocos esquemáticos de cada um dos operadores. Como os programas a fluxo de dados são concebidos na forma de grafos, foi decidido que eles seriam melhor iplemenatdos utilizando-se blocos esquemáticos, que permite uma representação visual do circuito. 
Estando prontos os blocos esquemáticos, foram implementados os comandos de controle de fluxo da linguagem $\mathrm{C}$ em grafos a fluxo de dados, trabalho esse que resultou na publicação de um artigo científico [Astolfi \& Silva 2007]. Por exemplo, entre vários outros, foi implementado o grafo correspondente aos comandos if-else, que será descrito em maiores detalhes a seguir, como exemplo. A declaração if-else é utilizada para expressar decisões. A Figura 7.4 ilustra um algoritmo simples que utiliza os comandos if-else, e seu respectivo grafo a fluxo de dados, que foi implementado, sob a forma de blocos esquemáticos, na ferramenta Xilinx ISE 8.2i.

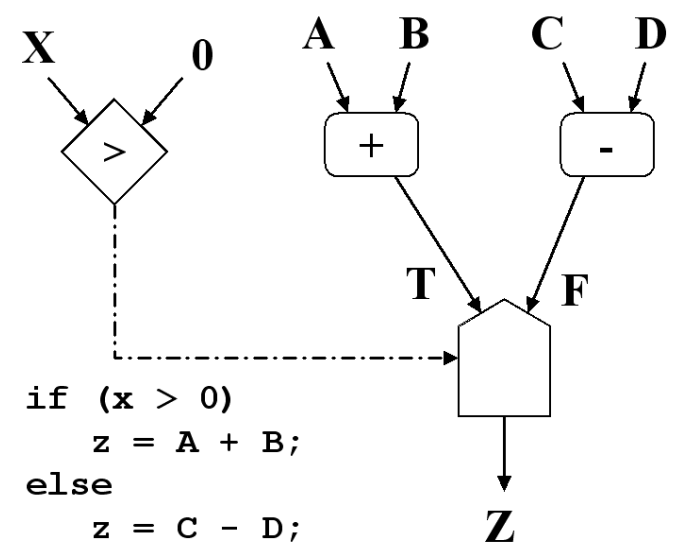

Figura 7.4 - Grafo a fluxo de dados extraído de um algoritmo que usa declarações if-else.

Para fins de teste, o grafo implementado foi simulado, utilizando-se o software Xilinx ISE Simulator, como mostra a Figura 7.5. No caso do grafo ilustrado na Figura 7.4, os sinais de entrada $a, b, c, d$, e $x$ assumiram respectivamente os valores $2,3,5,1$, e 2. Como dois é maior que zero, o valor obtido para $z$ foi 5 (2 adicionado a 3). Em um segundo experimento, foram atribuídos os valores $3,4,9,9$, e 0 aos mesmos sinais de entrada. Nesse caso, o valor resultante para $z$ foi zero (9 subtraído de 9), uma vez que o valor de x não é maior que zero.

Nos grafos implementados, e nos subseqüentes que serão apresentados, para que os dados de entrada e de saída fossem sincronizados, foi necessária a construção de dois blocos extras: INDATA, para a sincronização de um dado de entrada; e AOUT, que sincroniza o recebimento de um dado de saída. A Figura 7.6 mostra o grafo como ele foi implementado, no software ISE. 


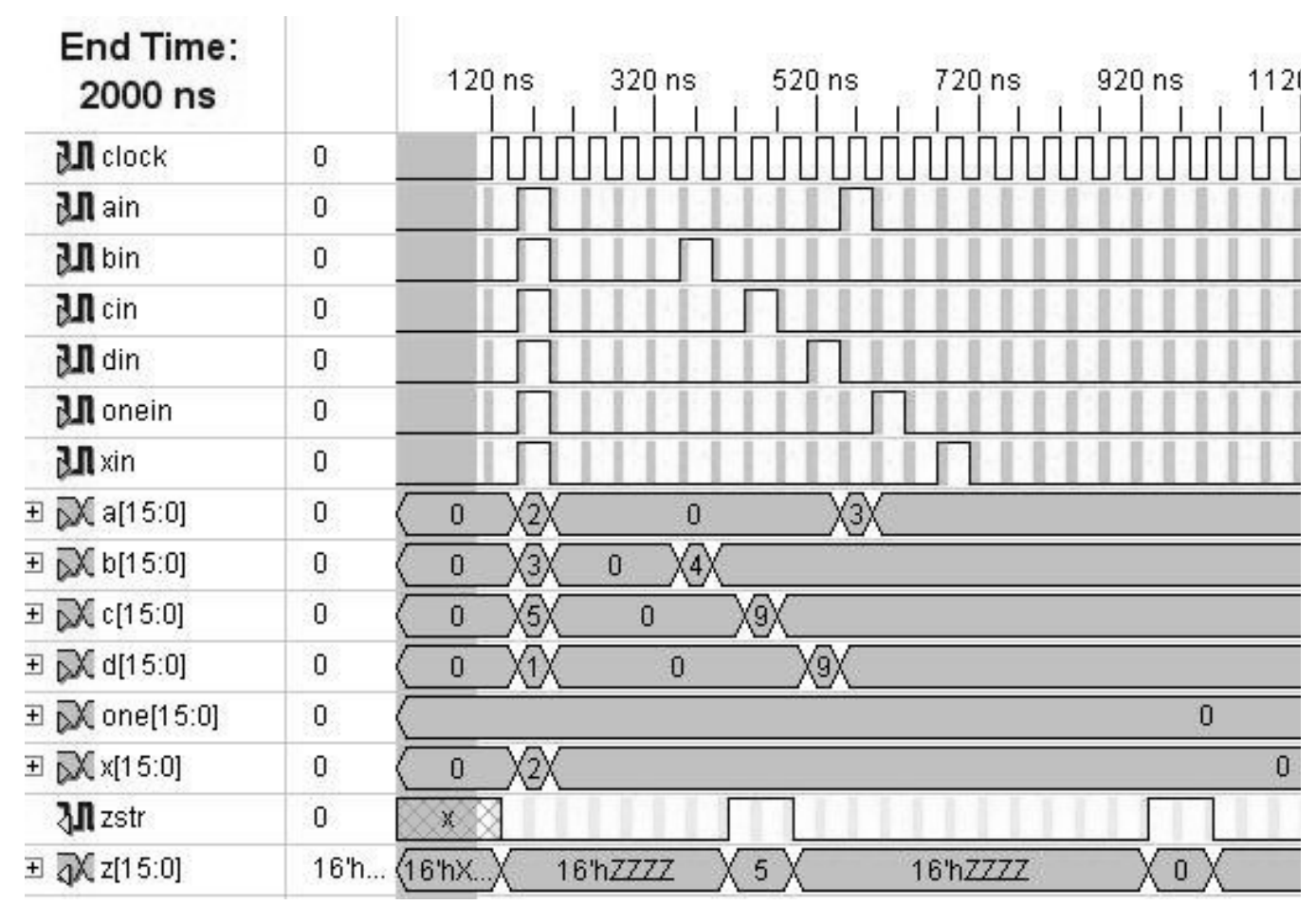

Figura 7.5 - Simulação do grafo if-else.

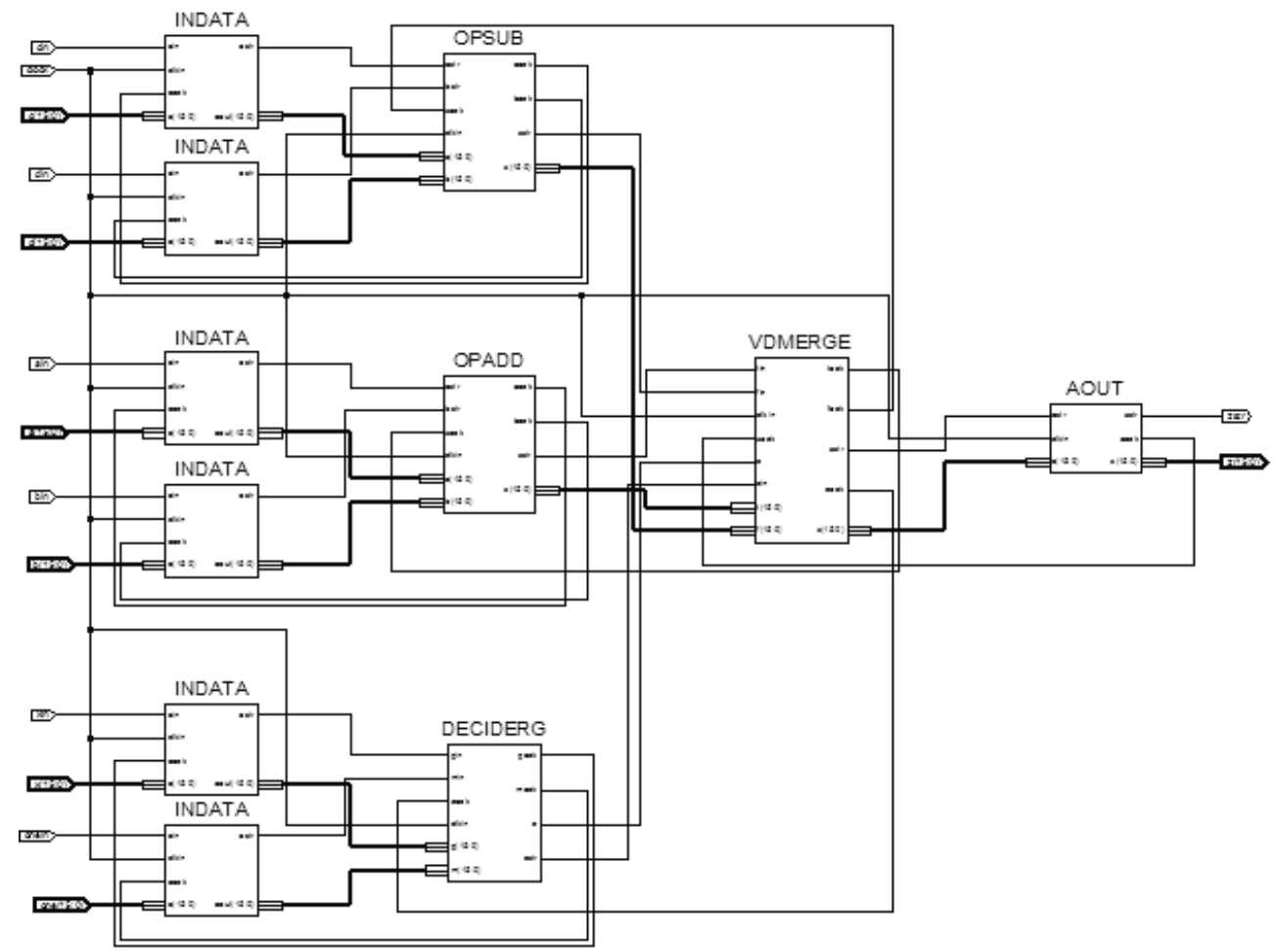

Figura 7.6 - Implementação, em blocos esquemáticos, do grafo "if-else". 


\subsection{Modelo a Fluxo de Dados Dinâmico}

Estando implementados e testados os operadores estáticos do modelo, finalmente foi possível adaptá-los para a versão dinâmica proposta no Capítulo 6. A Figura 7.7 ilustra um diagrama de máquina de estados que representa o operador OPADD dinâmico (OPADD_D), seu módulo parcialmente reconfigurável (PRM) OPADD_R, e o operador de junção nãodeterminística NDMERGE. Note que, na figura, não estão representados os multiplexadores, demultiplexadores e os bus macros necessários para haver comunicação entre os módulos. Cada retângulo com bordas arredondadas, que contém um conjunto de estados, representa um processo que será executado em paralelo junto aos demais, para aumento do desempenho. No caso dos operadores replicados, cada um deles é uma máquina de estados independente. Cabe ao controle de entrada decidir a qual operador é distribuído um token.

Pode-se ver que, na figura, o sistema está dividido em três partes distintas. Em OPADD_D e NDMERGE se concentra a parte estática, que fará parte do projeto-base necessário ao fluxo de projeto parcialmente reconfigurável. NDMERGE, como já foi dito, é utilizado para o envio de tokens ao operador subseqüente. OPADD_D é o operador OPADD implementado anteriormente acrescido de funções para o gerenciamento da reconfiguração parcial, além do envio de tokens ao PRM, que por sua vez, na figura, é o módulo OPADD_R.

Inicialmente, o operador OPADD_D, cujo bloco esquemático está ilustrado na Figura 7.8, se comporta da mesma maneira que o operador OPADD que o originou, aguardando por tokens em suas portas de entrada. Caso um token chegue a alguma de suas portas de entrada, como a, o sistema verifica o estado do registrador reca. Caso ele armazenar o valor "zero", o sistema se comporta como OPADD, armazenando o valor em um registrador ad e alterando o valor de reca para "um". Se inicialmente, entretanto, reca assumir o valor "um", o sistema verifica se OPADD_D tem alocado para si um módulo reconfigurável observando o valor do registrador reconf. Caso negativo, ele muda seu estado para solicita_config, que, por sua vez solicitará a reconfiguração, atribuindo "um" aos sinais rec e recstr (sendo esse ultimo o sinal de strobe). Como resposta, ele obtivera recstatus, que assume o valor "um" caso a reconfiguração tenha sido obtida com sucesso, e “zero" caso contrário. 


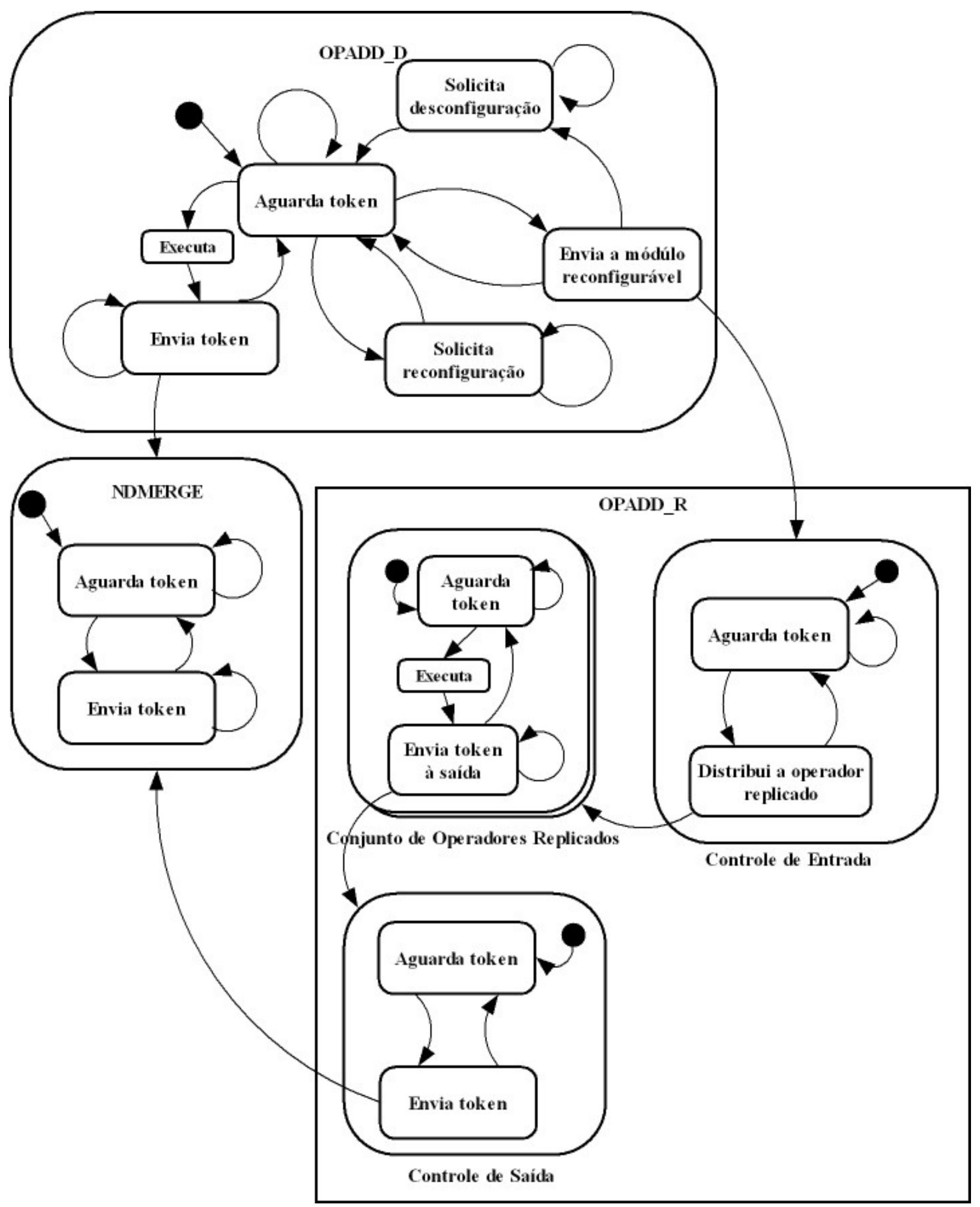

Figura 7.7 - Diagrama de máquina de estados, em notação SysML, representando a versão dinâmica de OPADD. 


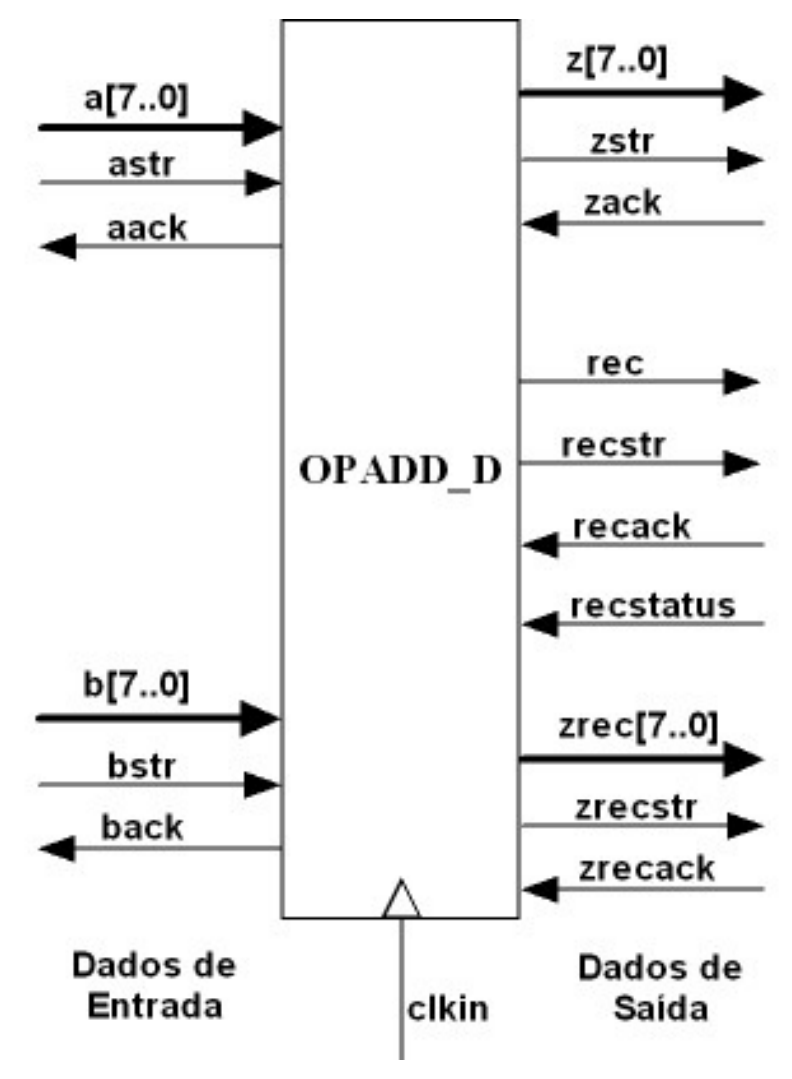

Figura 7.8 - Bloco esquemático de OPADD_D.

Caso o operador OPADD_D tenha alocado para si um módulo reconfigurável e ele receba um token em uma porta que já continha um token armazenado, ele verifica se é possível enviar o token a um operador replicado analisando o número de operadores replicados que já estão com a porta em questão ocupada por token. Se ainda restar operadores replicados com essa porta disponível, o sistema muda para o estado envia_para_modulo, e o token é enviado ao PRM utilizando-se os sinais zrec e zrecstr. Caso contrário, nada é feito, e o operador se comporta como se fosse estático. No estado envia_para_modulo, após a confirmação do envio, o sistema verifica se o número de tokens enviados de ambas as portas são iguais. Caso positivo, isso significa que para todos os tokens enviados em uma porta foram enviados seus tokens correspondentes na outra porta, tornando possível a solicitação da desconfiguração do módulo, e liberar sua respectiva região parcialmente reconfigurável para o PRM de outro operador. Dessa 
maneira, o estado é alterado para solicita_desconfig, no qual os sinais rec e recstr assumem os valores "zero" e "um", respectivamente.

Note que o operador OPADD_D armazena um token em cada uma de suas portas de entrada. Caso ele tenha um token armazenado e chegue seu token parceiro na outra porta, o disparo ocorre no próprio operador OPPAD_D, não havendo a necessidade, portanto, de operadores replicados. Devido às limitações de largura de barramentos nos bus macros, que suportam no máximo 8 bits, os barramentos e registradores utilizados em OPADD_D, assim como nos outros operadores implementados para o modelo parcialmente reconfigurável, tiveram a largura reduzida de 16 bits para 8 bits. O código-fonte de OPADD_D pode ser visto no Apêndice A.

Após sua implementação, foi necessário simular o operador criado para verificar se o mesmo funcionava da maneira esperada, como mostra a Figura 7.9, na qual são mostrados os resultados obtidos no ISE Simulator. Inicialmente, a porta de entrada a recebeu o token contendo o valor "1" (os valores zero devem ser ignorados, pois não há sinal de strobe ain). Dessa maneira, "1" é armazenado no registrador de a, e a porta é considerada "ocupada". Quando b recebe o valor "3", o operador pode ser disparado e o resultado "4" é enviado pela porta de saída, z, e as portas a e b são liberadas novamente, e a pode receber normalmente " 2 " em sua porta de entrada aproximadamente aos 400 ns. Quando se tentou, na posição aproximada de 700 ns, inserir mais um token na porta a, contendo dessa vez o valor "8", o operador muda para o estado de solicitar reconfiguração, uma vez que, por padrão, ele não possui nenhum módulo reconfigurável alocado. Assim, a partir da posição aproximada de 800 ns, são enviados sinais rec e recstr solicitando a reconfiguração. Enquanto não chega a resposta da solicitação nas entradas recack e recstatus, naturalmente o hipotético operador que enviou o token contendo o valor dois continuará reenviando-o até receber um sinal aack, indicando que nosso operador simulado recebeu o token com sucesso. Quando, finalmente, o operador recebe, nas entradas recack e recstatus a confirmação de que a reconfiguração foi feita com sucesso, uma vez que recstatus possui o valor um, é possível receber o valor "8" na porta a, cujo token será direcionado ao módulo reconfigurável através da saída zrec. Da mesma maneira, os valores "4" e "3" são recebidos e encaminhados ao PRM. Quando, por fim, aproximadamente na posição $1500 \mathrm{~ns}$, é recebido mais 
um token na entrada a, com o valor "1", o operador se comporta de maneira estática, pois todos seus três operadores replicados estão em uso, aguardando os parceiros dos dados armazenados nas portas a.

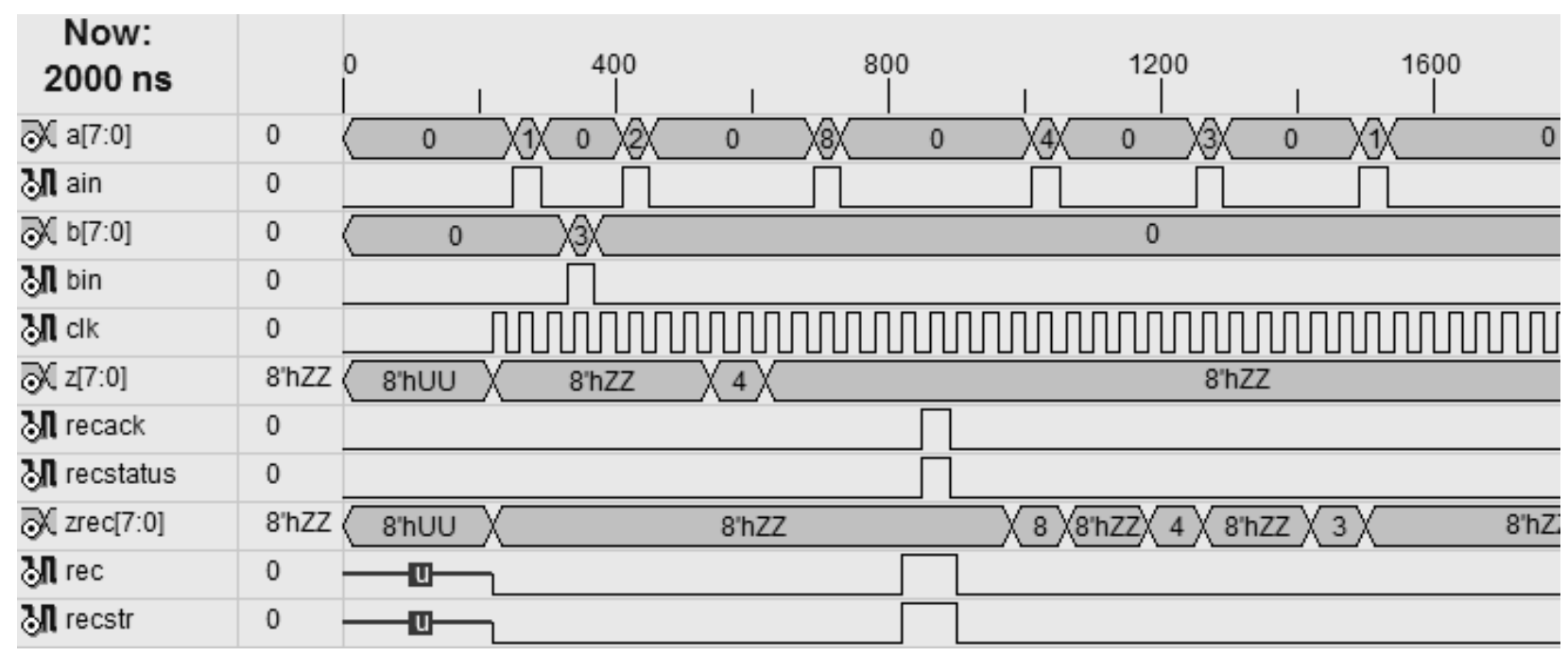

Figura 7.9 - Simulação do operador OPADD_D.

Após a implementação e teste de OPADD_D, concentrou-se o trabalho no desenvolvimento de OPPAD_R. Optou-se implementá-lo em um único arquivo de descrição de hardware, cujos sinais de entrada e saída estão representados no bloco esquemático ilustrado na Figura 7.10.

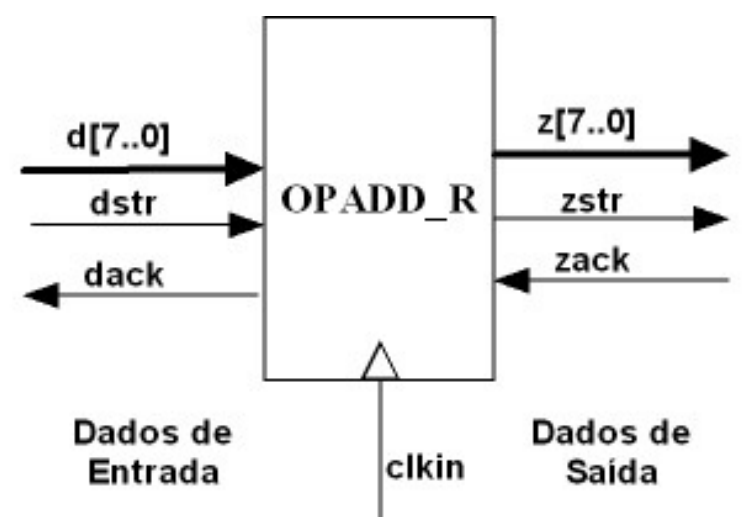

Figura 7.10 - Bloco esquemático de OPADD_R. 
Note que há apenas uma entrada de tokens, d, que receberá os valores que serão somados, além dos sinais de strobe de $\mathbf{d}$ e o de acknowledge de $\mathbf{z}$, que é o token de saída, contendo o resultado da adição. Note que são utilizadas apenas uma entrada e saída de tokens, para que fosse menos bus macros para comunicação entre o OPPAD_R e o resto da lógica estática fossem utilizados, simplificando assim a implementação do projeto, desde as especificações dos sinais que se conectarão a cada módulo, até o próprio posicionamento dos bus macros durante a fluxo de projeto parcialmente reconfigurável. Quando, no entanto, for implementado um protocolo de comunicação entre os diversos módulos do grafo a fluxo de dados, será necessário incorporar ao token informações adicionais especificando qual porta irá recebê-lo, em particular no módulo reconfigurável, para que se seja possível determinar em qual porta de entrada o token será alocado. Como não é o propósito deste trabalho desenvolver tal protocolo, nem mesmo a estrutura de matching proposta por Silva [2006], o módulo OPADD_R implementado não está ainda funcional em sua plenitude, sendo necessário modificá-lo, como trabalho futuro, para seu correto funcionamento. Da maneira como foi implementado, como os tokens não possuem nenhum tipo de identificação (tag), apenas um operador replicado está funcional. Por não haver nenhum tipo de identificação de tokens, foi definido, arbitrariamente, que os tokens recebidos seriam alocados de maneira alternada para a porta $\mathbf{a}$ e b, no operador replicado. $\mathrm{O}$ código-fonte de OPADD_D pode ser consultado no Apêndice B. Apesar de três operadores replicados terem sido definidos, apenas um deles é utilizado, devido à alocação alternada definida.

Para fins de teste, simulou-se o módulo criado no software ISE Simulator, e obtiveram-se os resultados apresentados na Figura 7.11.

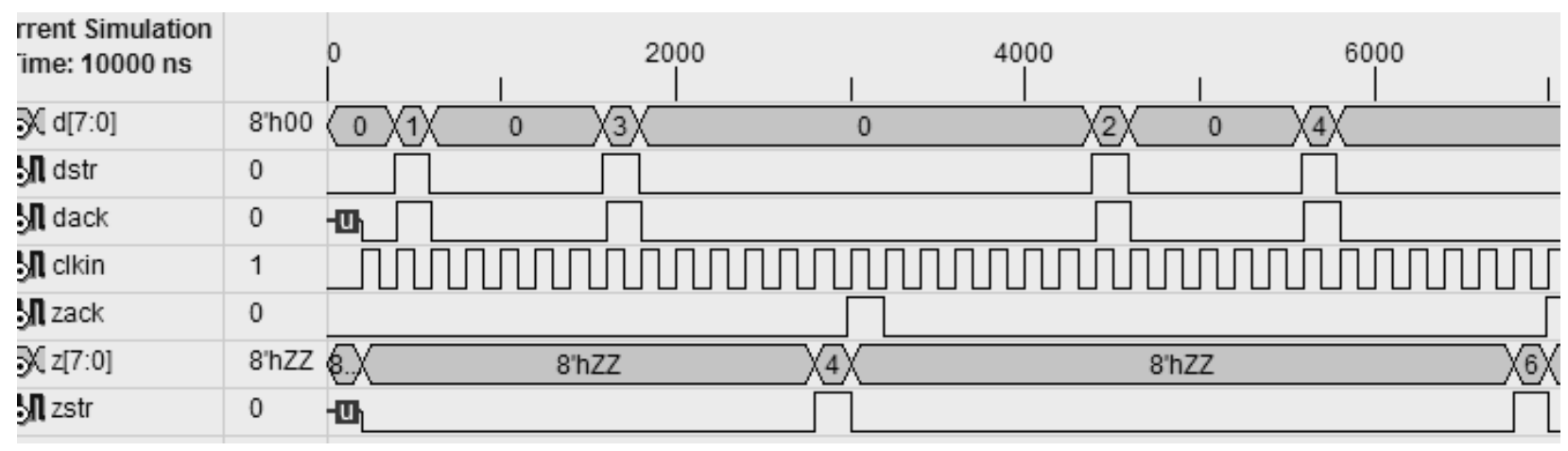

Figura 7.11 - Simulação de OPADD_R. 
Inicialmente, a entrada d recebeu um token contendo o valor um. Como se trata do primeiro token, o mesmo foi atribuído à porta a do primeiro operador replicado. O próximo token, com valor três foi atribuído à porta b. Estando disponíveis todos os tokens em cada porta de entrada, o operador replicado finalmente pôde ser disparado, obtendo-se assim o valor quatro, resultado da adição. Após seu disparo, o operador replicado foi liberado para receber novos tokens, dessa vez dois e quatro nas portas a e b, respectivamente. Obteve-se assim o valor seis, como esperado. A Tabela 7.1 mostra o sumário da utilização lógica de OPADD_D e OPADD_R.

Estando implementados o operador OPADD_D e seu respectivo OPADD_R, desenvolveu-se, de modo semelhante, o operador OPMULT_D, responsável pela multiplicação de dois tokens de entrada, e seu módulo OPMULT_R. Bastou, nesse caso, alterar o operador de adição para multiplicação nos códigos VHDL de OPADD_D e OPADD_R.

Finalmente, foram desenvolvidos o multiplexador MUX e o demultiplexador DEMUX, ambos de simples implementação. O operador de junção não-determinística NDMERGE já havia sido criado quando foram desenvolvidos os operadores estáticos. Nesse operador há apenas dois estados, aguarda_token e envia_token, sendo que no primeiro o módulo aguarda pela chegada de tokens de um operador dinâmico ou de seu PRM. Quando um token chega a uma porta de entrada, é repassado ao operador subseqüente no estado envia_token. Já os módulos MUX e DEMUX são multiplexadores e demultiplexadores clássicos, que recebem um sinal de entrada modulo e decidem, respectivamente, de qual operador e para qual operador deve ser encaminhado um conjunto de sinais, composto pelo token em si, e seus sinais de strobe e acknowledge.

Tabela 7.1 - Utilização lógica dos módulos OPADD_D e OPADD_R.

\begin{tabular}{l|lllll}
\hline Nome & Slices & $\begin{array}{l}\text { Slice Flip } \\
\text { Flops }\end{array}$ & $\begin{array}{l}\text { LUTs de } \\
\text { entrada }\end{array}$ & IOBs & TBUFs \\
\hline OPADD_D & 99 & 119 & 177 & 0 & 16 \\
OPADD_R & 47 & 83 & 64 & 0 & 8 \\
\hline
\end{tabular}




\section{8}

\section{Fluxo de Projeto para o Modelo Proposto}

Estando criados todos os operadores e módulos básicos para o desenvolvimento de um grafo, foi implementado, como prova de conceito para o modelo proposto, o grafo ilustrado na Figura 7.12. Neste exemplo, tem-se a saída $\mathbf{Z}=(\mathbf{A}+\mathbf{B}) \mathbf{x}(\mathbf{C}-\mathbf{1})$. Note que, para cada elemento de entrada do grafo há um módulo INDATA para sincronização dos dados, descrito anteriormente, na Seção 7.1.2, no Capítulo 7. Já na saída $\mathbf{Z}$, há um elemento AOUT, também descrito na mesma Seção, utilizado para sincronização dos dados de saída. Como o valor $\mathbf{C}$ é subtraído por um valor constante, não é necessário utilizar a versão dinâmica do operador de subtração OPSUB, porque, na realidade, só há uma fonte de tokens. Como OPSUB é estático, não é necessário um operador de junção não-determinística para o envio de seus dados. Têm-se, então, dois operadores dinâmicos, OPADD_D e OPMULT_D, e uma região parcialmente reconfigurável PRR, que receberá o PRM OPADD_R ou OPMULT_R. Como a região é compartilhada, é necessário um MUX e um DEMUX para o direcionamento dos tokens e seus sinais de strobe e acknowledge. Na fronteira entre a região estática (todo o sistema, exceto a PRR), e a parcialmente reconfigurável (a PRR em si), são necessários, para ser feita a comunicação entre essas regiões, dois conjuntos de três bus macros cada, que receberem nome de BM1 até BM6. Para que a figura fosse simplificada, só foram mostrados os sinais de strobe e 
acknowledge mais relevantes, apesar de existirem entre todos os módulos que transmitem dados entre si.

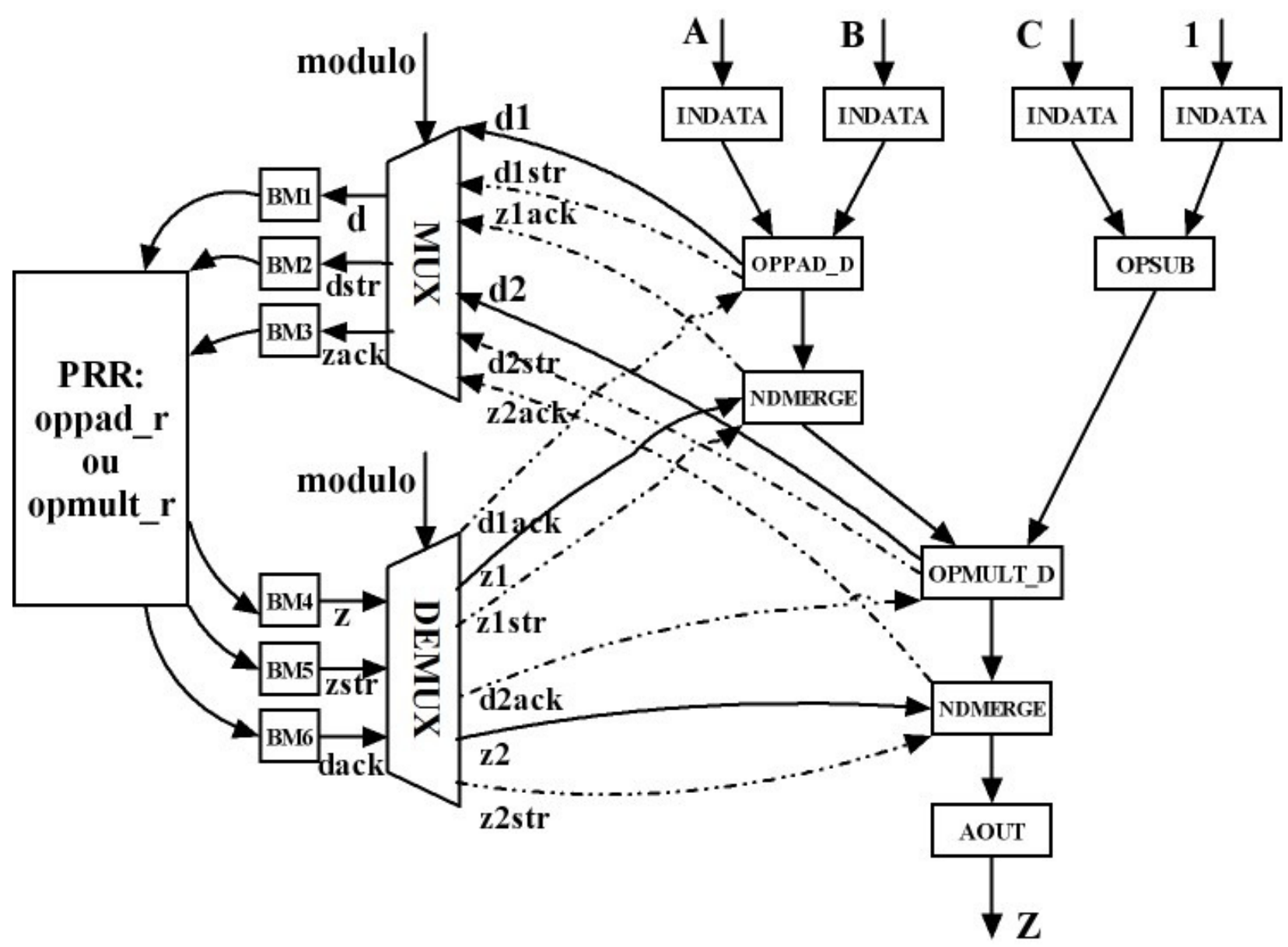

Figura 8.1 - Grafo implementado como prova de conceito inicial para o modelo proposto.

O fluxo de projeto parcialmente reconfigurável recomendado pelo early-access da Xilinx [Xilinx URL b] requer diversos passos que não são encontrados em um fluxo normal de projeto em FPGA, que envolve uma única passagem pelas ferramentas de implementação, isto é, a NGDBuild, a MAP e a PAR, cujo funcionamento será detalhado mais adiante. Já no caso do fluxo de projeto parcialmente reconfigurável há a necessidade de se implementar um projeto-base e cada PRM separadamente, e finalmente juntar o que foi obtido em um passo final. A Figura 8.2 ilustra os passos básicos do fluxo de projeto parcialmente reconfigurável. O primeiro passo envolve basicamente a codificação do projeto base e dos PRMs, cujas regiões de confinamento 
(constrains) serão definidas no segundo passo, utilizando-se o software PlanAhead. Já o mapeamento tecnológico (MAP) e o place \& route (PAR) da parte estática e dos PRMs serão feitos no terceiro passo, sendo os bitstreams finalmente gerados no quarto passo. Todo o projeto foi desenvolvido neste Capítulo teve como base a plataforma Virtex-II Pro, mas pode ser aplicado praticamente sem alterações a uma Virtex-4, que permite maior liberdade na definição das regiões de confinamento e bus macros.

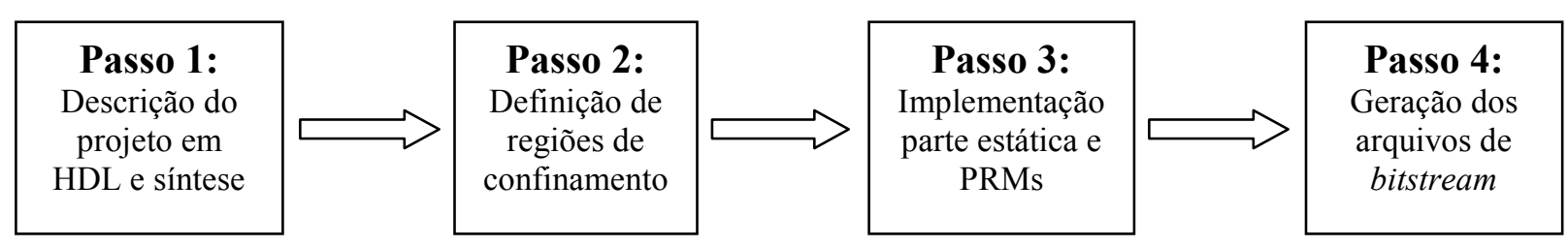

Figura 8.2 - Passos básicos do fluxo de projeto parcialmente reconfigurável.

\subsection{Primeiro passo: descrição do projeto em HDL e síntese}

Estando criados e testados todos os elemento do grafo a fluxo de dados a ser implementado, seguindo o fluxo de projeto dinamicamente reconfigurável, o primeiro passo foi a criação de um projeto "plano" (flat), inserido em um subdiretório chamado Flat. Neste projeto, não há a criação de netlists parciais, sendo o funcionamento do circuito semelhante ao de um sem reconfiguração parcial. Foi necessário criar um arquivo VHDL de topo (top.vhdl, que pode ser consultado no Apêndice C), que, seguindo a documentação da Xilinx, deve conter apenas:

- Instanciações de E/S (como as entradas A, B e C), descritas na região entity do código.

- Instanciações de primitivas de clock (no caso, o componente BUFGP, como mostra a Figura 8.3).

- Instanciações de módulos-base estáticos (como NDMERGE, INDATA, AOUT, OPADD_D, OPMULT_D, etc).

- Instanciação de um PRM para todos os PRR (no caso, OPADD_R para a única PRR). 
- Declarações de sinais de comunicação entre os módulos.

- Instanciações de bus macros. Neste projeto de topo, foram escolhidos bus macros do tipo busmacro_xc2vp_l2r_sync_narrow, estreitos (narrow), com direcionamento da esquerda para a direita, e síncronos, pelas vantagens oferecidas por este tipo (melhor sincronismo de comunicação, apesar do aumento da latência), sendo esse o recomendado na maior parte dos casos, excetuando-se naqueles em que o aumento da latência não é aceitável. $\mathrm{O}$ uso de bus macros largos (wide) seria mais vantajoso se fossem utilizados barramentos de maior largura, uma vez que bus macros desse tipo podem fornecer largura de banda de até 24 bits [Xilinx 2006a]. O direcionamento do bus macro, da esquerda para a direita, foi escolhido de maneira arbitrária, podendo ser também da direita para esquerda. O desenvolvimento do projeto, no entanto, seguiu o direcionamento que foi definido, como será visto a seguir. A Figura 8.4 mostra a instanciação do bus macro 1 (BM1), que liga o multiplexador à PRR. Foi necessário obter o bus macro necessário ao projeto (busmacro_xc2vp_l2r_sync_narrow.nmc), assim como os modelos de instanciação (busmacro_xc2vp_pkg.vhd), na página Partial Reconfiguration Early Access Tools Lounge [Xilinx URL b], e incorporar o arquivo de netlist (.nmc) ao diretório do projeto.

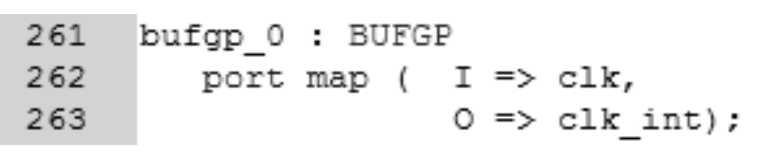

Figura 8.3 - Instanciação do clock, em nível de topo.

Para que fosse feita a comunicação entre o projeto parcialmente reconfigurável e um futuro Gerenciador de Reconfigurações (GR), descrito no Capítulo 6, foi necessário declarar no arquivo de topo um sinal modulo, que indica qual operador está utilizando o PRR em um determinado momento. Esse sinal será utilizado pelo MUX e pelo DEMUX. Além disso, foi necessário adicionar os sinais reca e recm, e seus respectivos sinais de strobe, acknowledge e status, para que fossem feitas as requisições de reconfiguração ou desconfiguração, como explicado na Seção 7.2 do Capítulo 7. A declaração desses sinais pode ser vista na Figura 8.5. No 
caso, a é o token de entrada na porta a, e ain é seu sinal de strobe. O mesmo se aplica a b, c, e d, sendo que nesse último o valor recebido será apenas um. Já z é o sinal de saída, e clk o de clock.

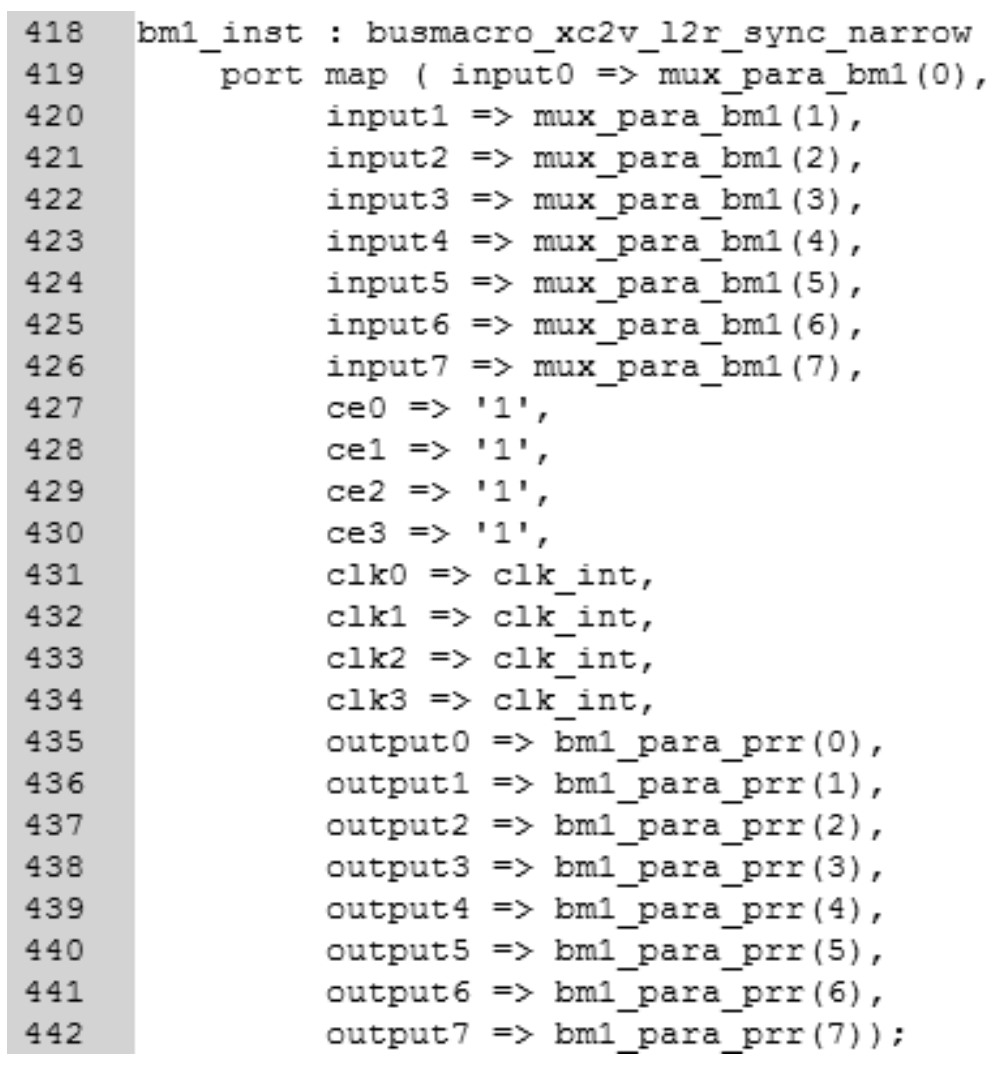

Figura 8.4 - Instanciação do bus macro 1 (BM1).

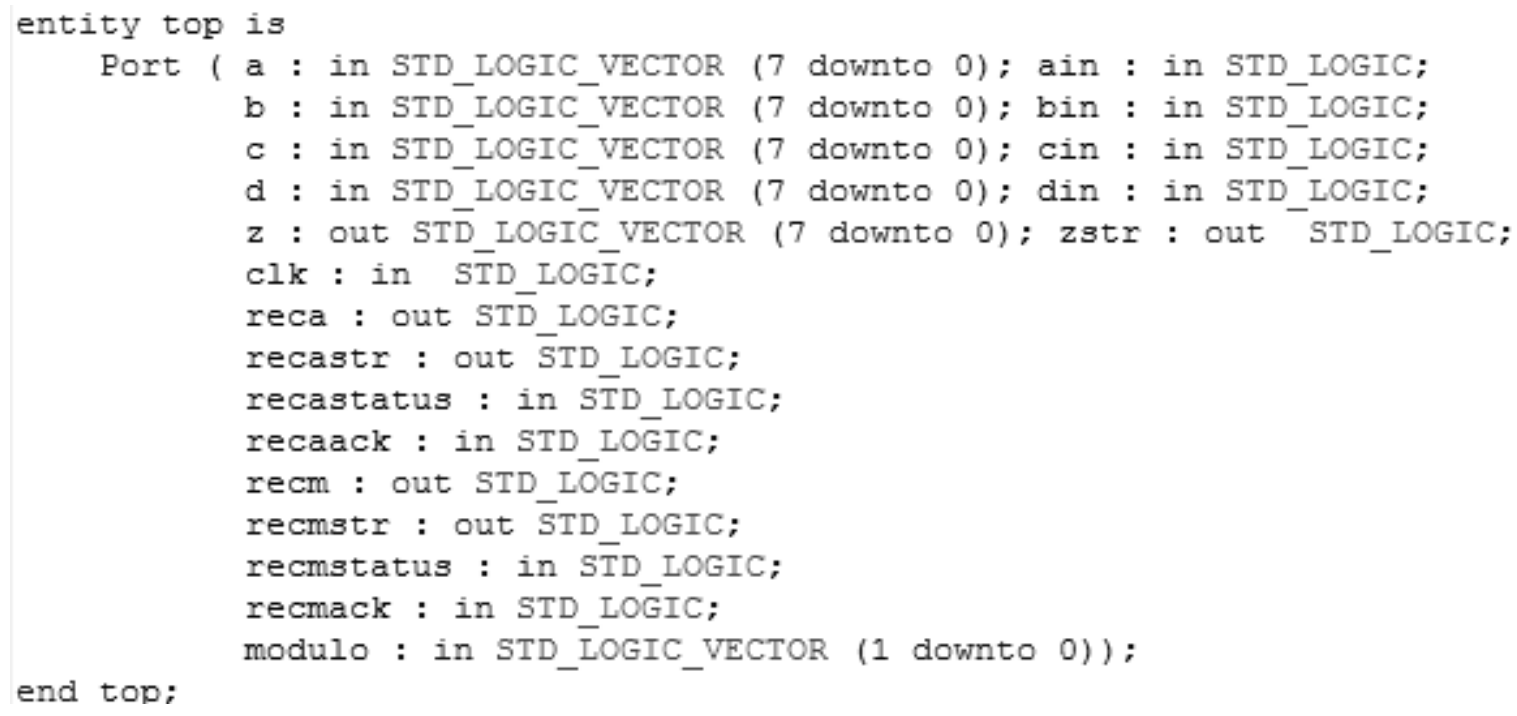

Figura 8.5 - Declaração dos sinais de entrada e saída do arquivo de topo do projeto. 
Note que esse arquivo de topo não contém nenhuma lógica, e que não é possível descrevê-lo utilizando-se blocos esquemáticos, sendo necessário para o processo de reconfiguração o uso de linguagem de descrição de hardware (HDL), no caso VHDL. Note também que a lógica estática está instanciada, assim como a de um módulo reconfigurável, que está instanciado para a região parcialmente reconfigurável (no caso, o OPPAD_R, como mostra a Figura 8.6). Todos os tipos de módulos reconfiguráveis (OPADD_R, OPMULT_R, etc.), precisam, em nível de sistema, ter o mesmo nome (MOD_RECONFIG).

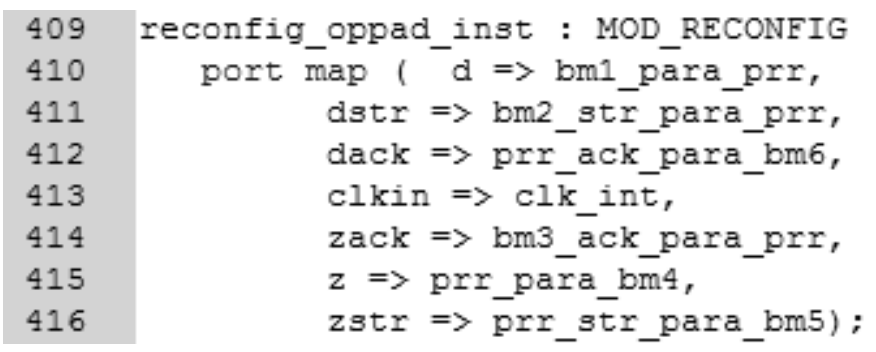

Figura 8.6 - Instanciação do módulo parcialmente reconfigurável (PRM).

Uma vez implementado o arquivo de topo, simulou-se o circuito por meio da utilização do Xilinx ISE Simulator, como mostra a Figura 8.7. Como os bus macros são incorporados como uma caixa-preta durante o processo de síntese, uma vez que seu código-fonte não está disponível, foi necessário obter no website da Xilinx e incorporar ao projeto um modelo de simulação em VHDL (busmacro_xc2vp_l2r_sync_narrow_model.vhd) para que fosse feita a simulação, uma vez que ele não é parte das bibliotecas de simulação padrão do unisim. Além disso, foi necessário definir cada entidade do circuito como "Simulation Only" para que a simulação fosse feita com sucesso.

Inicialmente, forneceu-se às entradas a, b, e c os valores “4", “3" e "2", respectivamente. $\mathrm{Na}$ entrada $\mathbf{d}$ foi sempre fornecido o valor " 1 ", por se tratar de uma constante. Como resultado, foi obtido o valor "7" na saída z, aproximadamente aos 800 ns. Quando a entrada a recebeu o valor "2", o registrador dessa porta já estava liberado, sendo o valor armazenado nele. Quando a entrada c recebeu o valor "8" (aos 400 ns) fez-se a subtração, e o valor "7" foi armazenado em uma porta do operador de multiplicação. Assim que o valor "3" chegou à porta a, foi solicitada a 
reconfiguração parcial n, sendo ela obtida com sucesso aos 800 ns, sendo os sinais recaack e recstatus, ambos com valor " 1 ", fornecidos manualmente. O envio desses sinais será, no futuro, feitos pelo GR. Assim, "3" foi enviado ao PRM e armazenado na porta a de um operador replicado. Quando o valor "1" chega à porta a, ele também é enviado ao PRM, dessa vez sendo armazenado na porta b do operador replicado porque, como foi visto na Seção 7.2 do Capítulo 7, os tokens são atribuídos às portas de um operador replicado de maneira alternada, porque não foi implementado um protocolo de comunicação e um circuito de matching. Assim, o operador replicado é disparado, e o valor resultante, “4", é enviado a OPMULT_D, que possui na outra porta o valor “7”, como vimos. Dessa maneira, na saída $\mathbf{z}$ tem-se o resultado "28”, como esperado.

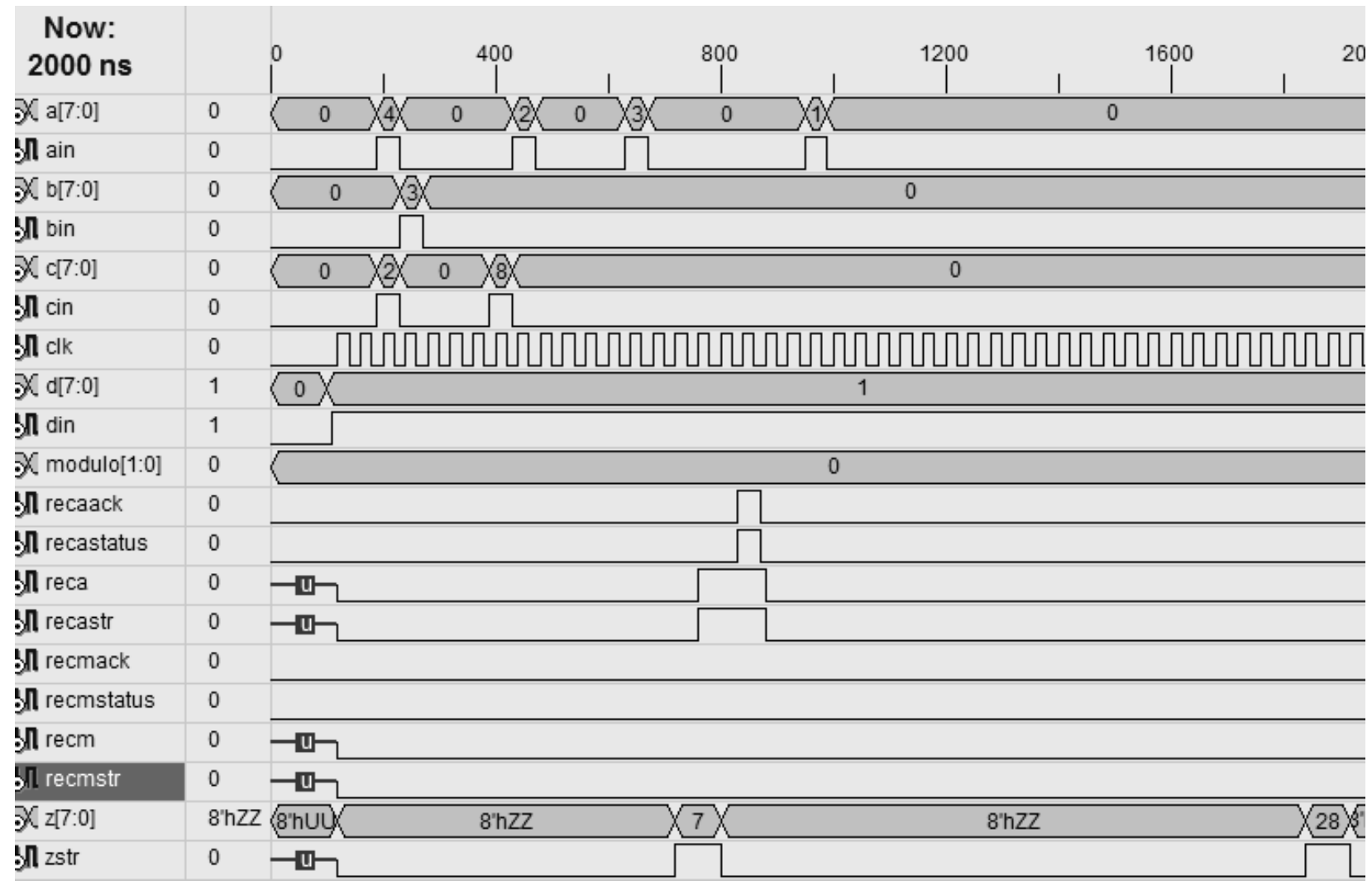

Figura 8.7 - Simulação do projeto descrito no nível de topo.

Após a construção de um projeto "plano" (circuito_flat), o fluxo de projeto da Xilinx recomenda que seja sintetizado cada um dos módulos, inclusive os reconfiguráveis. Criou-se um 
novo subdiretório chamado Synth, contendo por sua vez três outros subdiretórios: top, static, reconfig (tendo oppad e oppmult como subdiretórios), como mostra a Figura 8.8. Dessa maneira, foram sintetizados todos os módulos em seus respectivos diretórios. Ao contrário dos módulos de menor nível, foi necessário sintetizar o módulo de topo com inserções de buffers de entrada e saída. Os arquivos de netlist dos bus macros são inseridos no diretório Data.

Na Tabela 8.1 é possível ver o sumário da utilização lógica do projeto plano em uma Virtex-II Pro modelo xc2vp30-7ff896.

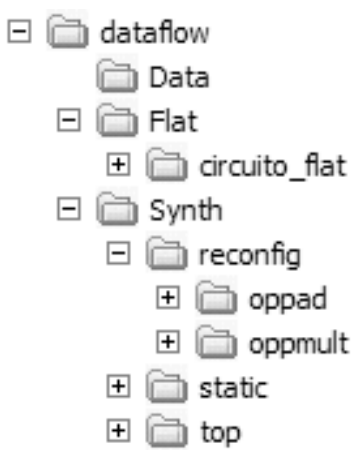

Figura 8.8 - Estrutura de diretórios do projeto.

Tabela 8.1 - Sumário da utilização lógica de um dispositivo Virtex-II Pro pelo projeto plano.

\begin{tabular}{l|lll}
\hline Tipo de Lógica & Usadas & Disponíveis & Utilização \\
\hline Slices & 410 & 13696 & $2 \%$ \\
Slice Flip Flops & 551 & 27392 & $2 \%$ \\
LUTs & 690 & 27392 & $2 \%$ \\
\hline
\end{tabular}

\subsection{Segundo passo: definição das regiões de confinamento dos elementos do projeto}

Estando criadas as netlists de cada elemento do projeto, para que fosse definida cada região de confinamento (constrains) desses elementos, além das demais fases do fluxo de projeto 
dinamicamente reconfigurável, decidiu-se pela utilização do software Xilinx PlanAhead, devido à facilidade de uso, se comparado aos demais softwares utilizados para o mesmo fim, como o PACE ou o Floorplanner. Inicialmente, criou-se um novo projeto no PlanAhead, chamado project_1. Durante o processo de criação do projeto, como mostra a Figura 8.9, foram importadas as netlists criadas anteriormente. No caso do módulo reconfigurável, importou-se apenas o módulo referente ao OPPAD_R. Uma vez criado o projeto, foi necessário defini-lo como parcialmente reconfigurável, selecionando o item Set PR Project, no menu File.

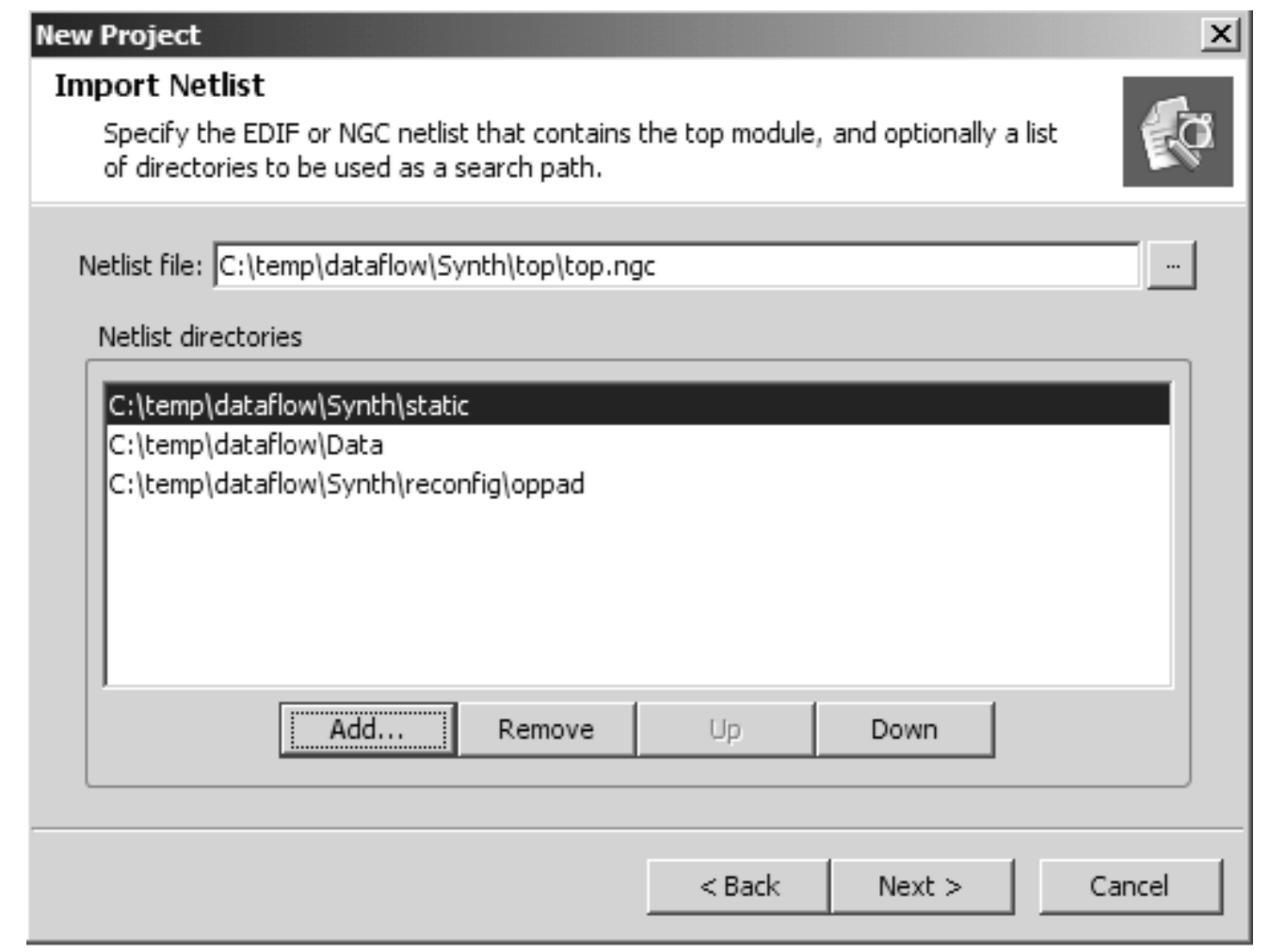

Figura 8.9 - Importação de netlists durante criação de projeto no PlanAhead.

Em seguida, todos os módulos estáticos, não reconfiguráveis, foram selecionados e criouse um novo Pblock, que foi chamado static (Figura 8.10). No módulo reconfig_oppad_inst, escolheu-se a opção Draw Pbloc, e suas bordas foram delimitadas entre os slices X12Y112 e X31Y143. Dessa maneira, um novo PBlock foi definido, chamado pblock_reconfig, sendo esse reconfigurável, ao contrario do static. 
Para que fosse possível definir o posicionamento dos bus macros, escolheu-se a opção Create Site Constraint Mode, na barra de tarefas do PlanAhead. Foi escolhido cada bus macro no diretório Primitives no painel de netlists, e posicionou-se os bus macros 1, 2, e 3 no lado esquerdo do Pblock, enquanto os demais foram posicionados no lado direito, como mostra a Figura 8.11. Esse posicionamento é necessário porque foram escolhidos apenas bus macros com direcionamento de dados da esquerda para a direita. Como os três primeiros são de entrada, é necessário colocá-los à esquerda, já nos três últimos, ocorre o oposto. Note que os bus macros precisam ficar exatamente na fronteira entre a região estática e a reconfigurável. Além dos bus macros, foi necessário também definir a posição dos buffers de entrada e saída descritos no arquivo de topo, como $a$, ain, $b$, bin, $c$, cin, $z$, zstr, reca, recastr, recaack, recastatus, etc.

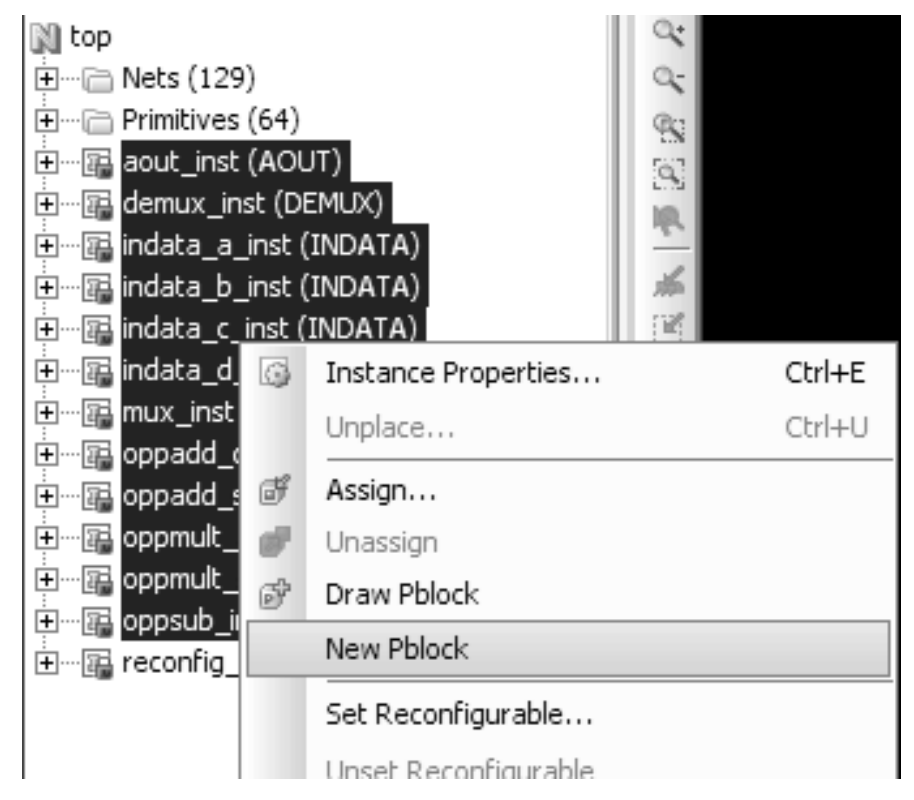

Figura 8.10 - Criação de um Pblock para os módulos estáticos.

Após a definição de cada PBlock, o próximo passo foi definir o PBlock pblock_reconfig como reconfigurável. Para isso, selecionou-se esse PBlock e escolheu-se a opção Set Reconfigurable no menu. O nome escolhido para o módulo foi oppad. Após isso, surgiu uma nova região reconfigurável no painel ExploreAhead Runs, que será utilizado mais à frente. Como existe, além do OPPAD_R, mais um módulo reconfigurável que pode estar na região reconfigurável definida, OPPMULT_R, foi necessário adicionar mais um módulo reconfigurável. 
Para isso, bastou clicar no PBlock e escolher a opção Add Reconfigurable Module. Após a definição do nome como oppmult, foi escolhido o arquivo de netlist dentro do diretório Synth $\backslash$ reconfig $\backslash$ loppmult. Uma vez adicionado o módulo, esse se tornou o módulo ativo do projeto, isto é, o que será carregado por padrão durante a configuração inicial do FPGA.

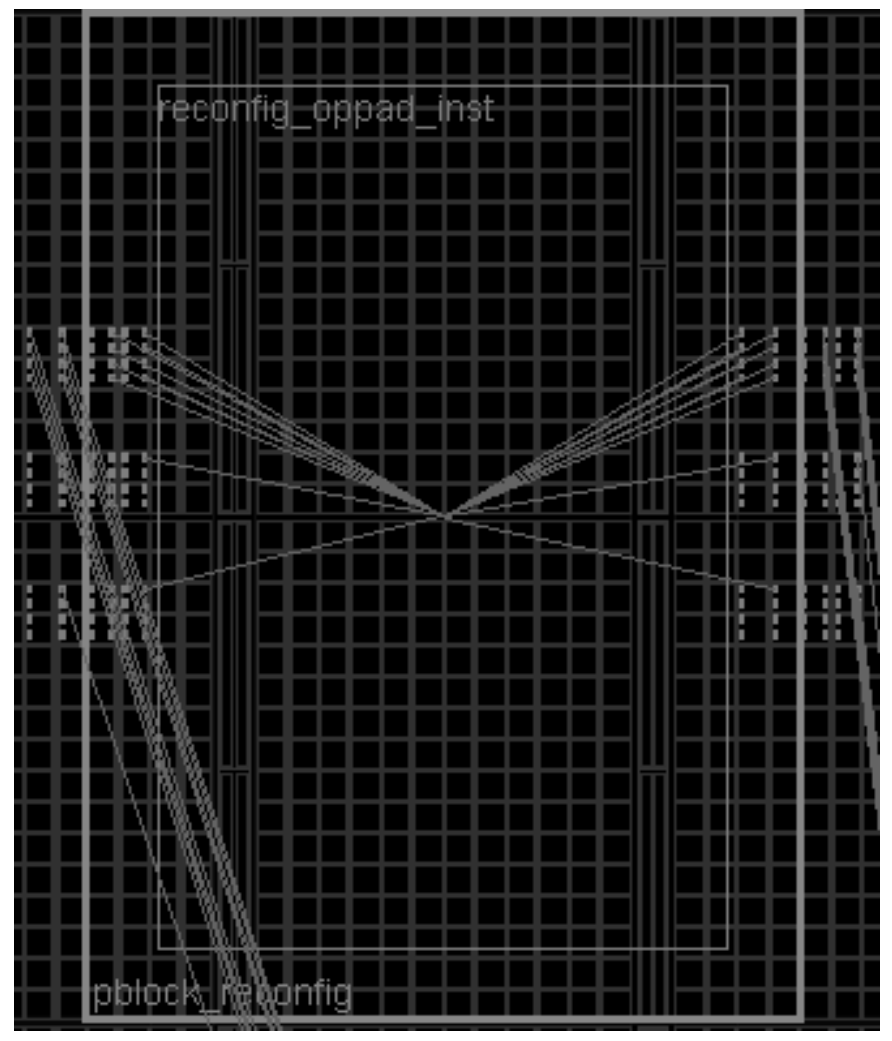

Figura 8.11 - Pblock no qual a PRR é confinada e seus bus macros correspondentes.

\subsection{Terceiro passo: implementação do projeto estático e dos módulos reconfiguráveis}

Este passo se resume basicamente no mapeamento tecnológico (MAP) e place \& route (PAR), inicialmente de cada módulo estático do projeto, incluindo o projeto de base (top). Por fim, cada módulo parcialmente reconfigurável será também implementado. Estes procedimentos são necessários para a posterior criação dos bitstreams estáticos e parciais, que serão utilizados para a programação do FPGA. Esta fase do projeto só pôde ser completada quando se adquiriu o 
software Xilinx ISE Foundation 9.1i, uma vez que o que havia disponível (Xilinx ISE Webpack 9.2i) não dava suporte apropriado à execução do comando NGDBuild, que lê arquivos de netlists e cria um arquivo .ngd descrevendo o projeto lógico, necessário para o MAP e o PAR dos módulos reconfiguráveis e do projeto estático.

Antes de continuarmos, foi necessário verificar se havia algum erro, utilizando-se o Design Rule Check (DRC), do PlanAhead. Vários erros, causados pela ausência de definição de posição de buffers de E/S, e pela posição incorreta do PRR, foram corrigidos. Ao selecionarmos a região reconfigurável e a aba statistics no painel Pblock Properties, é possível verificar a estimativa de uso da região por cada módulo reconfigurável. Caso algum tipo de elemento lógico de algum deles utilizasse mais de cem por cento da região definida, seria necessário expandir o tamanho do PBlock reconfigurável. As Figuras 8.12 e 8.13 mostram, respectivamente, a utilização do PRR pelos PRMs OPMULT_R e OPADD_R.

Physical Resources Estimates
\begin{tabular}{|l|r|r|r|}
\hline Type of Site & Available & Required & $\%$ Utilization \\
\hline LUT & 1280 & 60 & 4.69 \\
\hline FF & 1280 & 75 & 5.86 \\
\hline SLICE & 640 & 46 & 7.19 \\
\hline MULT18X18 & 8 & 1 & 12.50 \\
\hline RAMB16 & 8 & 0 & 0.00 \\
\hline TBUF & 320 & 8 & 2.50 \\
\hline
\end{tabular}

Figura 8.12 - Porcentagem de uso de cada elemento lógico por OPMULT_R no PRR.

\begin{tabular}{|l|r|r|r|}
\hline \multicolumn{1}{|c|}{ Type of Site } & Available & Required & \% Utilization \\
\hline LUT & 1280 & 60 & 4.69 \\
\hline FF & 1280 & 83 & 6.48 \\
\hline SLICE & 640 & 51 & 7.97 \\
\hline MULT18X18 & 8 & 0 & 0.00 \\
\hline RAMB16 & 8 & 0 & 0.00 \\
\hline TBUF & 320 & 8 & 2.50 \\
\hline
\end{tabular}

Figura 8.13 - Porcentagem de uso de cada elemento lógico por OPADD_R no PRR.

Após a verificação de todos os aspectos mencionados, pôde-se finalmente gerar os arquivos .ncd, resultantes do mapeamento tecnológico e do place \& route. Utiliza-se para isso o 
painel ExploreAhead Runs, como mostra a Figura 8.14. Inicialmente, é escolhida a implementação estática (static), e foi executado o comando Launch Runs. Uma vez terminado o processo, são escolhidos ambos módulos reconfiguráveis (reconfig_oppad_inst_oppad e reconfig_oppad_inst_oppmult) e foi utilizado o mesmo comando.

\begin{tabular}{|c|c|c|c|c|c|}
\hline \multicolumn{6}{|c|}{ ExploreAhead Runs } \\
\hline$\nabla$ & Name & Flow & Strategy & Status & Progress \\
\hline \multirow{5}{*}{ 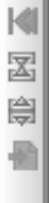 } & 曰. 圆 floorplan_1 & & & & \\
\hline & 盾 static & PR 9 & Static Defaults & PAR Complete! & $100 \%$ \\
\hline & 回 pblock_reconfig_oppad & & & & \\
\hline & ... $\vec{\square}$ reconfig_oppad_inst_oppad & PR 9 & PR Module Defaults & PAR Complete! & $100 \%$ \\
\hline & ... $\vec{\square}$ reconfig_oppad_inst_oppmult & PR 9 & PR Module Defaults & PAR Complete! & $100 \%$ \\
\hline
\end{tabular}

Figura 8.14 - O painel ExploreAhead Runs, utilizado durante o processo de MAP, PAR e geração de bitstreams.

\subsection{Quarto passo: geração dos arquivos bitstream}

Estando implementadas as lógicas estática e parciais, bitstreams parciais e totais podem finalmente ser gerados. Um arquivo de bitstream total é um projeto completo e funcional, que junta um módulo reconfigurável à lógica estática. Para cada módulo reconfigurável, é gerado um bitstream total, além do parcial.

As ferramentas utilizadas são a PR_verifydesign e PR_assemble, próprias para o processo de reconfiguração parcial. No PlanAhead, bastou escolher a opção Run PR Assemble no menu do painel ExploreAhead Runs. Dessa maneira, como mostra a Figura 8.15, finalmente os bitstreams (BIT) foram gerados no diretório project_1 $\backslash$ project_1.runs $\backslash$ floorplan_1 $\backslash$ merge, estando prontos para serem utilizados para a programação do FPGA. Em um PC com processador single-core, de $2 \mathrm{GHz}$, e $1 \mathrm{~GB}$ de memória RAM, o processo levou no total quatro minutos e três segundos. Na Tabela 8.2 pode-se verificar o tamanho, em bytes, dos bistreams de cada PRM, além do bitstream total. 
Com a geração dos arquivos de bitstream, finalmente é possível programar o FPGA, inicialmente com um bistream total, havendo posteriormente a possibilidade de reconfiguração parcial, durante a execução do FPGA, utilizando-se uma interface de configuração, como SelectMAP, JTAG, ou ICAP.

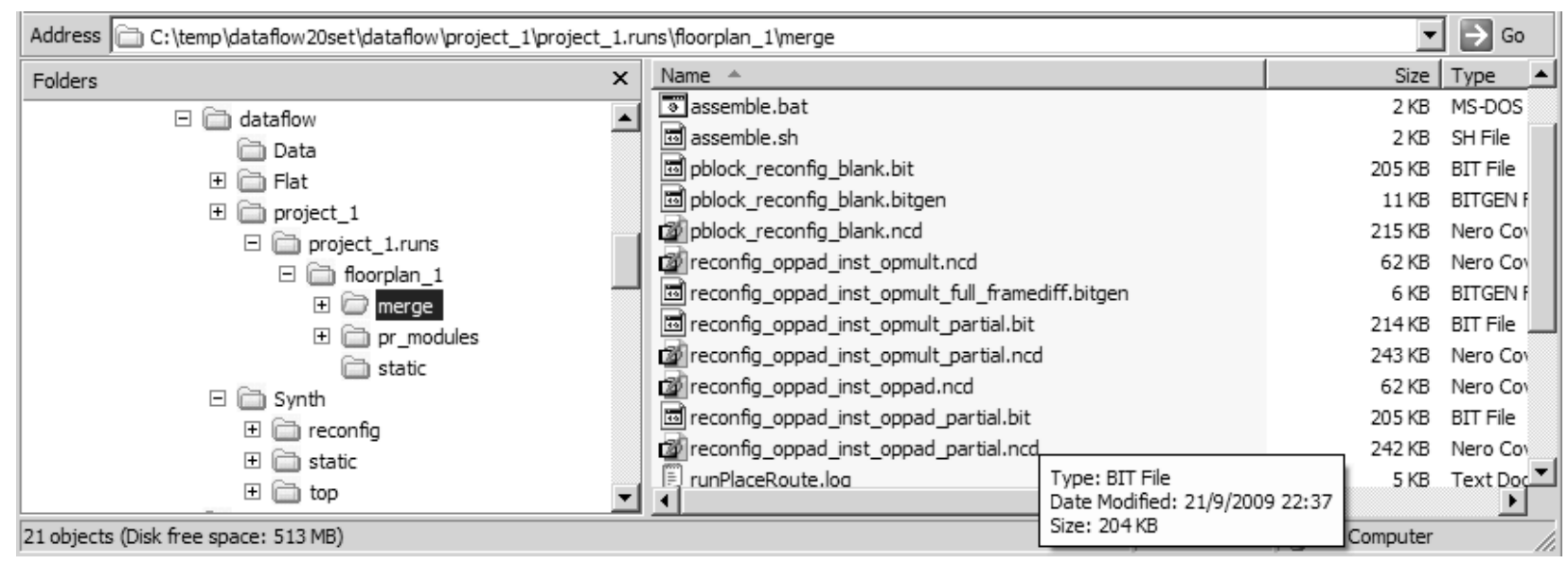

Figura 8.15 - Arquivos bitstream gerados, incluindo os parciais de cada PRM.

Tabela 8.2 - Tamanho dos bitstreams gerados.

\begin{tabular}{l|l}
\hline Arquivo bitstream & Tamanho em bytes \\
\hline Bitstream total (área estática e reconfigurável) & 1.448 .817 \\
Bitstream reconfigurável parcial opadd & 209.219 \\
Bitstream reconfigurável parcial opmult & 218.908 \\
Bitstream reconfigurável parcial em branco & 209.207 \\
\hline
\end{tabular}

\subsection{Avaliação do overhead da reconfiguração parcial}

O tempo levado pelo processo de configuração de uma PRR é diretamente proporcional ao tamanho do arquivo de bitstream utilizado para configurar o FPGA, que, por sua vez, é influenciado pelas dimensões das PRRs definidas, assim como a quantidade e o tipo dos 
operadores ou subgrafos incluídos no PRM, como mostra a Tabela 8.2. Durante o processo de reconfiguração parcial, o overhead de tempo de transmissão dos dados não pode ser ignorado, ainda mais se considerarmos que apenas um bitstream pode ser programado no FPGA de cada vez, então, se mais de um operador ou subgrafo requisitar a reconfiguração ao Gerenciador de Reconfigurações, apenas um dele conseguirá, e os outros necessariamente se comportarão de modo estático durante o período de reconfiguração do primeiro.

No processo de reconfiguração dinâmica, o bitstream parcial é segmentado e transferido de uma memória fora do chip a uma memória de um periférico OPB (On-chip Peripheral Bus), onde ele é escrito, um segmento por vez, na porta ICAP. Como esses processos de escrita são operações exclusivas, o tempo de reconfiguração baseada em módulos pode ser estimado pela equação a seguir [Sedcole 2006].

$$
T r \cong \frac{S b}{S f} \times\left(\frac{1}{t}+\frac{1}{w}\right)
$$

Nesse caso, é considerada a utilização da porta ICAP, cujo acesso pode ser controlado por software em um processador embarcado. Na equação, $\operatorname{Tr}$ é o tempo de reconfiguração, em segundos, $S b$ é o tamanho do bistream, e $S f$ o tamanho do quadro (frame) de comunicação, $t$ é a taxa na qual um quadro é transmitido de um dispositivo de memória fora do chip para o periférico $\mathrm{OPB}$, e $w$ a taxa na qual um quadro é escrito na porta ICAP durante o processo de reconfiguração.

Supondo, por exemplo, a configuração em uma Virtex-II Pro modelo xc2vp7 baseada na arquitetura Sonic-on-a-Chip, que utiliza quadros de 424 bytes. Nesse dispositivo, as taxas $t$ e $w$ são de aproximadamente 7,86 e 117 quadros por milissegundo, respectivamente [Sedcole 2006]. Substituindo os valores na fórmula, obtém-se um $T r$ igual a aproximadamente $70 \mathrm{~ms}$ para a reconfiguração parcial do PRM OPADD_R, que possui tamanho de 209.219 bytes, o que nos permite concluir que a reconfiguração parcial está limitada pela transmissão de dados através da plataforma, e deve ser usada estritamente quando necessário. 


\section{9}

\section{Conclusões e Trabalhos Futuros}

Após a apresentação de conceitos básicos sobre o modelo a fluxo de dados, mais especificamente a relação entre operadores e arcos em um grafo a fluxo de dados, foram apresentados os conceitos de laços iterativos e reentrância em grafos. Em seguida, apresentou-se algumas arquiteturas básicas de processadores a fluxo de dados e arquiteturas atuais baseadas em elementos a fluxo de dados, destacando-se neste último caso a arquitetura WaveScalar. Passou-se então à descrição da proposta da ferramenta ChipCflow para a execução de algoritmos diretamente em hardware no modelo de arquitetura a fluxo de dados dinâmica, onde foram apresentados o modelo de instâncias, estrutura tagged-token e os operadores propostos na ferramenta.

Estando descrita a ferramenta ChipCflow, apresentou-se aspectos básicos sobre FPGA, suas estruturas internas, e o modo de reconfiguração. Em seguida, foram apresentadas algumas arquiteturas que usam esses conceitos. Especial atenção foi dada aos modelos reconfiguração parcial dinâmica baseada em módulos ou diferença, no qual se definiu a reconfiguração baseada em módulos como o modelo utilizado pela ChipCflow.

Como parte do processo de reconfiguração na ferramenta ChipCflow, foram apresentados quatro passos para a execução de reconfiguração parcial dinâmica na ferramenta, sendo eles: $1^{\circ}$. 
descrição do projeto em HDL e síntese; $2^{\circ}$. definição das regiões de confinamento dos elementos do projeto; $3^{\circ}$. implementação do projeto estático e dos módulos reconfiguráveis; $4^{\circ}$. geração dos arquivos bitstream. Finalmente, foi feita uma avaliação de desempenho da reconfiguração parcial.

Este trabalho, portanto, se concentrou no aprendizado e domínio de técnicas para a reconfiguração parcial dinâmica nos FPGAs da Xilinx, aplicada ao modelo a fluxo de dados dinâmico particular à ferramenta ChipCflow. Foi apresentado um modelo que contempla a criação e execução de instâncias, em forma de operadores, de programas a fluxo de dados dinâmico específico da ferramenta, além de uma prova de conceito (proof of concept) para esse modelo, cuja validação foi feita por meio de simulações e verificações executadas em softwares apropriados da Xilinx. Como resultados deste trabalho foram obtidos os bitstreams necessários à reconfiguração parcial do FPGA.

Considerou-se, no processo de desenvolvimento deste projeto, inicialmente o aprendizado das características e limites da reconfiguração parcial, para finalmente ser possível a proposição de um modelo para o grafo a fluxo de dados dinâmico que permitisse a criação e execução de instâncias de operadores, tendo como base o modelo de instâncias proposto por Silva [2006]. Como não é possível criar, com o uso das técnicas atuais, uma arquitetura completamente flexível e reconfigurável, decidiu-se pela utilização da versão estática do grafo a fluxo de dados como um projeto-base, ao qual estão ligados os operadores replicados, em módulos parcialmente reconfiguráveis, o que torna dinâmica a execução da máquina a fluxo de dados. Dessa maneira, no decorrer deste trabalho foram desenvolvidos os operadores do grafo a fluxo de dados dinâmico, tendo como base a versão estática deles, além de módulos auxiliares necessários à implementação de um grafo ou programa funcional, utilizado como prova de conceito para o modelo e operadores propostos. Apesar de, nesse grafo implementado como prova de conceito, não haver vantagens em utilizar a versão dinâmica dos operadores, uma vez que o algoritmo é simples, não contendo nenhum tipo de reentrância, a implementação teve estritamente o propósito de prova de conceito. A partir deste trabalho e do desenvolvimento de um modelo de comunicação utilizando tags para identificação dos tokens, além de um método de partição de grafos, um Gerenciador de Reconfigurações, e um compilador para a ferramenta ChipCflow, será 
possível implementar um algoritmo mais complexo que poderá se beneficiar do modelo a fluxo de dados dinâmico.

Apesar do desenvolvimento deste projeto ter como base a plataforma Xilinx Virtex-II Pro, seria possível aplicá-lo praticamente sem alterações a uma Virtex-4, que possui um fluxo de projeto igual ao da Virtex-II Pro, apesar de permitir maior liberdade na definição das regiões de confinamento das regiões parcialmente reconfiguráveis e dos bus macros. A escolha da Virtex-II Pro se deu pelo fato de haver uma disponível para o grupo de pesquisa do ChipCflow, apesar de ela não ter sido usada neste projeto. Na plataforma Virtex-5, entretanto, o suporte à reconfiguração parcial é limitado [Xilinx URL b].

\subsection{Principais Contribuições}

Entre as principais contribuições deste trabalho ao projeto ChipCflow podem ser destacadas as seguintes.

- O entendimento do fluxo de projeto parcialmente reconfigurável, possibilitando a geração de bitstreams parciais que serão utilizados por um futuro Gerenciador de Reconfigurações (GR), responsável pelo gerenciamento da programação do FPGA por esses bitstreams.

- O modelo proposto de reconfiguração parcial para o ChipCflow, que poderá ser utilizado como base para o desenvolvimento de um protocolo de comunicação na forma de tagged-tokens, um circuito de matching, e finalmente o GR, necessários para o funcionamento pleno do modelo, o que possibilitará uma análise na forma de benchmarking, comparando os tempos de execução obtidos tanto no modelo estático quanto no dinâmico. Uma vantagem do modelo proposto é sua versatilidade, podendo assumir características tanto do modelo estático quanto o dinâmico, dependendo da disponibilidade de regiões reconfiguráveis para replicação de operadores ou subgrafos.

- Os operadores desenvolvidos, tanto estáticos quanto dinâmicos, assim como os módulos parcialmente reconfiguráveis, além dos módulos adicionais, que poderão 
ser utilizados em outros modelos propostos no futuro, caso o apresentado não apresente resultados satisfatórios, após a análise de benchmarking.

Parte do trabalho apresentado, especificamente no que refere aos operadores e grafos estáticos implementados, deu origem a uma publicação [Astolfi \& Silva 2007]. Os resultados obtidos ao final do desenvolvimento deste trabalho serão ainda submetidos para publicação.

\subsection{Limitações}

Como já dito, devido à impossibilidade de flexibilidade total no que se refere à reconfiguração parcial, a principal limitação do modelo apresentado é o compartilhamento das regiões parcialmente reconfiguráveis (PRR) por um conjunto de operadores ou subgrafos, cujas replicações não podem ocupar uma mesma PRR em um determinado momento.

Outra limitação do modelo apresentado é um gargalo na região entre o multiplexador (MUX) e o demultiplexador (DEMUX), incluindo nela os bus macros e a PRR, que impossibilita o envio de dois ou mais tokens de um determinado operador aos seus operadores replicados em um mesmo ciclo de clock. Os controladores de entrada e saída dentro dos módulos parcialmente reconfiguráveis (PRM, em inglês) também ilustram esse gargalo, porque eles só podem lidar com um token em um determinado ciclo de clock. Uma solução para essa limitação seria o uso de controladores, multiplexadores e demultiplexadores para cada porta de entrada ou saída em um operador, e não para todo o operador, o que demandaria mais lógica e a inclusão de um maior número de bus macros.

O tempo de geração dos bitstreams pode também ser um problema, no caso do ChipCflow. Esse processo pode ser demorado, uma vez que é necessário gerar para todos os elementos que podem ser replicados, ainda mais se considerarmos todas as combinações de subgrafos, cujos bitstreams dos PRMs precisam ser gerados com antecedência, durante o fluxo de projeto.

Como mostrou a Seção 8.5, o tempo levado para a transmissão dos bitstreams durante o processo de reconfiguração parcial se mostrou insatisfatório na plataforma Virtex-II. Isso, porém, é uma limitação tecnológica que pode ser atenuada com o tempo. Por exemplo, a largura de 
banda de reconfiguração de uma Virtex-4 é oito vezes maior que o da Virtex-II, além de possibilitar PRRs menores, o que pode diminuir o tamanho do bistream [Wang et al. 2007].

Não foi possível testar a programação dos bitstreams gerados durante o desenvolvimento deste projeto em uma FPGA Virtex, uma vez que nenhuma estava disponível para a implantação deste projeto, cujo desenvolvimento se baseou em simulação, além do uso de ferramentas para validação do projeto, como o DRC do PlanAhead, e a própria geração com sucesso dos arquivos bitstream. Entretanto, o processo de teste prático seria relativamente simples, bastando utilizar o software iMPACT incorporado ao Xilinx ISE. Inicialmente, seria escolhido um bitstream completo para a configuração total do dispositivo. Para que fosse carregado um bitstream parcial, bastaria configurar o FPGA com o mesmo, por meio da utilização do mesmo software.

\subsection{Trabalhos Futuros}

Devido às limitações apresentadas, deverá ser feita a análise em nível de compilação para a determinação da distribuição das PRRs aos operadores ou subgrafos potencialmente replicáveis, evitando dessa maneira conflitos que tornam estática uma parte do algoritmo a fluxo de dados, porque os elementos replicados de duas regiões do grafo (no caso deste projeto, dois operadores) não podem ocupar uma mesma PRR ao mesmo tempo.

Dentro dos PRMs, com o aumento do número de instâncias de grafos replicados, serão utilizados multiplexadores e demultiplexadores para o envio de tokens do controlador de entrada e ao controlador de saída, melhoria essa que diminuirá o número de sinais de comunicação necessários dentro do PRM.

Existe a possibilidade de se adaptar este modelo para a replicação de subgrafos, e não apenas de operadores individuais, para que mais de um operador do grafo a fluxo de dados possa utilizar uma determinada PRR, tornando assim mais eficiente o processo de atribuição de PRRs às diversas partes de um grafo, uma vez que o número de operadores de um grafo geralmente será muito maior que o número de PRRs. 
Como já foi dito, para termos um modelo completamente funcional, suportando reconfigurações dos PRRs de maneira dinâmica, será necessário implementar um circuito de matching, um protocolo de comunicação entre os operadores, onde cada novo token que circule pelo grafo contenha informações (tags) que o identifiquem, além do próprio Gerenciador de Reconfigurações (GR). Quando o modelo funcionar de maneira plena, será possível analisá-lo por meio benchmarks, comparando-o com um modelo puramente estático. A arquitetura-alvo de hardware endereçada pelo GR ainda não está completamente definida, podendo ser implementada completamente em FPGA, o que compromete a flexibilidade do sistema; ou em um processador incorporado à Virtex, como um Power PC ou Microblaze, para o qual se deve considerar a questão crítica da comunicação hardware-software. Da maneira como foram desenvolvidos, os operadores dinâmicos sempre demandam a configuração do dispositivo quando eles precisam de uma PRR ou quando eles deixam de utilizá-la. Isso pode sobrecarregar a comunicação entre o hardware e software, no GR, de maneira inaceitável. Uma melhoria, portanto, seria um mecanismo para solicitação esporádica de configuração.

Outra melhoria que poderá ser feita futuramente é a inclusão de lógica que permitiria a atribuição de mais de uma PRR a um operador ou subgrafo, o que atenuaria a principal limitação do modelo, que é a impossibilidade de se atribuir mais de uma PRR a um operador dinâmico. Esse melhor compartilhamento de PRRs poderia ser feito utilizando-se multiplexadores e demultiplexadores adicionais, todos controlados pelo GR. 


\section{Referências Bibliográficas}

Arul, J.; Yeh, T.; et al. (2005), “An Efficient Way of Passing of Data in a Multithreaded Scheduled Dataflow Architecture". In Eighth International Conference on High-Performance Computing in Asia-Pacific Region, pp. 492-497, 2005.

Arvind; Nikhil, R.S. (1990), "Executing a Program on the MIT Tagged-Token Dataflow Architecture". IEEE Transactions on Computers, vol. 39, pp. 300-318, 1990.

Arvind (2005), "Dataflow: Passing the token". ISCA Keynote, 2005.

Astolfi, V.F.; Silva, J.L. (2007), "Execution of algorithms using a Dynamic Dataflow Model for Reconfigurable Hardware - Commands in Dataflow Graph". In The III IEEE Southern Conference on Programmable Logic, pp. 225-230, 2007.

Atmel Corp., URL: http://www.atmel.com/products/FPGA/ <Acesso em outubro de 2009>.

Bolsens, I. (2006), "Programming Modern FPGAs". Technical Talks and Presentations MPSOC'06, Xilinx University Program, 2006.

Borkar, S. (2007), “Thousand core chips: a technology perspective". In Proceedings of the $44^{\text {th }}$ annual Design Automation Conference, San Diego, California, pp. 746-749, 2007.

Boyd, S.; Dornfeld, D.; et al. (2007), "Environmental Challenges for 45-nm and 32-nm node CMOS Logic”. 2007 IEEE International Symposium on Electronics \& the Environment, pp. 102105, maio de 2007.

Brown, S.; Vranesic, Z. (2005), "Fundamentals of Digital Logic with VHDL Design", 2a edição, McGraw-Hill. 2005. 
Burger, D.; Keckler, S.W.; et al. (2004), "Scaling to the End of Silicon with EDGE Architectures". IEEE Computer, vol. 37, ed. 7, pp. 44-55, julho de 2004.

Callahan, T.J.; Hauser, J.R.; Wawrzynek, J. (2000), "The Garp architecture and C compiler". IEEE Computer, vol. 33, ed. 4, pp. 62-69, abril de 2000.

Cardoso, J.M.P. (2000), “Compilação de Algoritmos em Java para Sistemas Computacionais Reconfiguráveis com Exploração do Paralelismo ao Nível das Operações”. Tese para obtenção de grau de Doutor, Universidade Técnica de Lisboa, 2000.

Cardoso, J.M.P.; Neto, H.C. (2003), “Compilation for FPGA-Based Reconfigurable Hardware”. IEEE Design Test of Computers Magazine, vol. 20, ed. 2, pp. 65-75, março-abril de 2003.

Celoxica Inc., URL: http://www.celoxica.com/<Acesso em outubro de 2009>.

Collins, L. (2003), “Chip Makers Hit Heat Barrier”. In IEEE Review, vol. 49, ed. 1, pp. 22-23, janeiro de 2003 .

Compton, K.; Hauck, S. (2002), "Reconfigurable Computing: A Survey of Systems and Software”. In ACM Computing Surveys, vol. 34, ed. 2, pp. 171-210, junho de 2002.

Costa, K.A.P. (2007), "Execução de Algoritmos Utilizando o Modelo a Fluxo de Dados Dinâmico para Hardware Reconfigurável - Ferramenta de Conversão C em Grafo a Fluxo de Dados". Monografia de qualificação de tese para obtenção de grau de doutor, Universidade de São Paulo, fevereiro de 2007.

Costa, K.A.P. (2009), "Uma ferramenta para execução de algoritmos utilizando o modelo a fluxo de dados dinâmico em hardware reconfigurável para a arquitetura ChipCflow: módulo de conversão C em grafo a fluxo de dados". Tese para obtenção de grau de Doutor, Universidade de São Paulo, julho de 2009. 
Dennis, J.B.; Misunas, D.P. (1975), “Preliminary Architecture for a Basic Data-Flow Processor”. In Proceedings of the 2nd Annual Symposium on Computer Architecture, vol. 3, ed. 4, pp. 126132, janeiro de 1975.

Dennis, J.B. (1980), “Dataflow Supercomputers”. In IEEE Computer, pp. 48-56, novembro de 1980.

Dorairaj, N.; Shiflet, E.; Goosman, M. (2005), "PlanAhead Software as a Platform for Partial Reconfiguration". Xilinx Inc., Xcell Journal, 2005.

Ebeling, C.; Cronquist, D. C.; Franklin, P. (1996), "RaPiD - Reconfigurable Pipelined Datapath". In International Workshop on Field-Programmable Logic and Applications, pp. 126$135,1996$.

El-Araby, E.; Gonzalez, I.; El-Ghazawi, T. (2007), "Performance bounds of partial run-time reconfiguration in high-performance reconfigurable computing". Proceedings of the 1 st international workshop on High-performance reconfigurable computing technology and applications, Reno, Nevada, pp. 11-20, 2007.

Flynn, M.; Hung, P. (2005), "Microprocessor Design Issues: Thoughts on the Road Ahead". In IEEE Micro, vol. 25, ed. 3, pp. 16-31, 2005.

Gajski, D. D.; Padua, D. A.; et al. (1982), "A Second Opinion on Dataflow Machines and Languages". In IEEE Computer, vol. 15, ed. 2, pp. 58-69, 1982.

Gelsinger, P.P. (2001), "Microprocessors for the New Millennium: Challenges, Opportunities, and New Frontiers".In IEEE Solid-State Circuits Conference, pp 22-25, 2001.

Gokhale, M.; Stone, J.; et al. (2000), "Stream-oriented FPGA computing in the Streams-C high level language”. 2000 IEEE Symposium on Field-Programmable Custom Computing Machines (FCCM), pp. 49, 2000. 
Goldstein, S.C.; Schmit, H.; Budiu, M.; et al. (2000), "PipeRench: A Reconfigurable Architecture and Compiler”, In IEEE Computer, vol. 33, ed. 4, pp. 70-77, 2000.

Gupta, S.; Dutt, N.; et al. (2003), "SPARK: A High-Level Synthesis Framework For Applying Parallelizing Compiler Transformations". Proceedings of the 16th International Conference on VLSI Design, pp. 461-466, 2003.

Gurd, J.R.; Kirkham C.C.; Watson, I. (1985), “The Manchester prototype dataflow computer”. Communications of the ACM, vol. 28, ed. 1, pp. 34-52, janeiro de 1985.

Hauck, S.; Fry, T.W.; et al. (2004), "The Chimaera reconfigurable functional unit". IEEE Transactions on Very Large Scale Integration (VLSI) Systems, vol. 12, ed. 2, pp. 206-217, fevereiro de 2004.

Hwang, K.; Briggs, F. (1984), “Computer Architecture and Parallel Processing”. McGraw-Hill Book Company, 1984.

Hwang, K. (1993), “Advanced Computer Architecture: Parallelism, Scalability, Programmability". McGraw-Hill Inc., 1993.

Intel (2005), Documentação Técnica. Intel Developer Forum Spring 2005, março de 2005.

Kao, C. (2005), “Benefits of Partial Reconfiguration”. Xilinx, Inc, 2005.

Kavi, K.M.; Giorgi, R.; Arul, J. (2001), "Scheduled dataflow: execution paradigm, architecture, and performance evaluation”. In Transactions on Computers, vol. 50, ed. 8, pp. 834-846, agosto de 2001 .

Köhler, S.; Schirok, J.; et al. (2008), "Efficiency of Dynamic Reconfigurable Datapath Extensions - A Case Study". In Proceedings of the 4th international workshop on Reconfigurable Computing, pp. 300-305, 2008. 
Laskowski, E.; Olejnik, R.; et al. (2004), "Scheduling byte code-defined data dependence graphs of object oriented programs". In International Conference on Parallel Computing in Electrical Engineering, pp. 398-401, 2004.

Lee, B.; Hurson, A.R. (1993), "Issues in Dataflow Computing”. Academic Press, New York, NY, Estados Unidos, 1993.

Lee, W.; Barua, R.; et al. (1998), "Space-time scheduling of instruction-level parallelism on a raw machine". ACM SIGOPS Operating Systems Review, vol. 32, pp. 46-57, dezembro de 1998.

Lopes, J.J.; Silva, J.L.; et al. (2006), “A Benchmark Approach for Compilers in Reconfigurable Hardware". In the 6th International Workshop on System on Chip for Real Time Applications, pp. 120-124, 2006.

Lopes, J.J. (2007), “Estudos e Avaliações de Compiladores para Arquiteturas Reconfiguráveis”. Dissertação para obtenção de grau de Mestre, Universidade de São Paulo, maio de 2007.

Ling, X.P.; Amano, H. (1993), "WASMII: a data driven computer on a virtual hardware”. In EEEE FPGAs for Custom Computing Machines, pp. 33-42, 1993.

Lysaght, P.; Blodget, B.; et al. (2006), "Invited Paper: Enhanced Architectures, Design Methodologies and CAD Tools for Dynamic Reconfiguration of Xilinx FPGAs”. In International Conference on Field Programmable Logic and Applications, 2006. FPL '06, pp. 1-6, agosto de 2006.

Mai, K.; Paaske, N.; et al. (2000), "Smart Memories: A Modular Reconfigurable Architecture”. In The 27th International Symposium on Computer Architecture, pp. 161-171, junho de 2000.

Mei, B.; Vernalde, S.; Verkest, D.; et al. (2003), “ADRES: An Architecture with Tightly Coupled VLIW Processor and Coarse-Grained Reconfigurable Matrix". In International Conference on Field-Programmable Logic and Applications, vol. 2778, pp. 61-70, 2003. 
Mercaldi, M. (2005), "Development of a WaveScalar Performance Model”. Dissertação de Mestrado, Universidade de Washington, 2005.

Mermoud, G. (2004), "A Module-Based Dynamic Partial Reconfiguration Tutorial”. École Polytechnique Fédérale de Lausanne, Suiça, novembro de 2004.

Mirsky, E.; DeHon, A (1996), "MATRIX: a reconfigurable computing architecture with configurable instruction distribution and deployable resources". In IEEE Symposium on FieldProgrammable Custom Computing Machines, pp. 157-166, 1996.

Mishra, M.; Goldstein, S. C. (2007), "Virtualization on the Tartan Reconfigurable Architecture". In International Conference on Field-Programmable Logic and Applications, pp. 323-330, 2007.

Moore, G.E. (1965), "Cramming more components onto integrated circuits". Electronics Magazine, pp. 56-59, 1965.

Moore, G.E. (1975), "Progress in digital integrated electronics". Electron Devices Meeting, pp. $1-13,1975$.

Moseley R. (2002), “Transcending Static Deployment of Circuits: Dynamic Run-Time Systems and Mobile Hardware Processes for FPGAs". Tese para obtenção de grau de Doutor, Universidade de Kent, Canterbury, setembro de 2002.

Nascimento, P.S.B, Lima, M.E., et al. (2006), "Sistemas Reconfiguráveis: Novo paradigma para o desenvolvimento de aplicações de computação massiva de dados". I Jornada Científica da UNIBRATEC, novembro de 2006.

O'Reilly, T. (2007), “Google's Folding@Home on the 'Multi-Core Crisis"'. O’Reilly' Radar, junho de 2007. URL: http://radar.oreilly.com/archives/2007/06/googles_folding.html <Acesso em outubro de 2009>. 
Papadopoulos, G.M.; Culler, D.E. (1990), "Monsoon: an explicit token-store architecture". In Proceedings of the 17th Annual International Symposium on Computer Architecture, pp. 82-91, junho de 1990.

Parkhurst, J.; Darringer, J.; et al. (2006), "From Single Core to Multi-Core: Preparing for a new exponential". In International Conference on Computer Aided Design, pp. 67-72, novembro de 2006.

Pedroni, V. (2004), “Circuit Design with VHDL”, The MIT Press, 2004.

Pellerin, D.; Thibault, S. (2005), "Practical FPGA Programming in C", Editora Prentice Hall PTR, 2005.

Pollack, F. (1999), "New Microarchitecture Challenges in the Coming Generations of CMOS Process Technologies". In the 32nd annual ACM/IEEE international symposium on Microarchitecture, 1999.

Razdan, R.; Smith, M.D. (1994), "A High-Performance Microarchitecture with HardwareProgrammable Functional Units". In Proceedings of the 27th Annual International Symposium on Microarchitecture, pp. 172-180, novembro de 1994.

Ribeiro, A.A.L. (2002), "Reconfigurabilidade dinâmica e remota de FPGAs". Dissertação para obtenção de grau de Mestre, Universidade de São Paulo, julho de 2002.

Ronen, R.; Mendelson, A.; et al. (2001), "Coming Challenges in Microarchitecture and Architecture". In Proceedings of the IEEE, vol. 89, ed. 3, pp. 325-340, março de 2001.

Sankaralingam, K.; Nagarajan, R.; et al. (2003), "Exploiting ILP, TLP, and DLP Using Polymorphism in the TRIPS Architecture". In the 30th Annual International Symposium on Computer Architecture (ISCA), vol. 23, ed. 6, pp. 422-433, junho de 2003. 
Scalera, S.M.; Vazquez, J.R. (1998), "The design and implementation of a context switching FPGA”. In the IEEE Symposium on FPGAs for Custom Computing Machines, Napa Valley, EUA, pp. 78-85, 1998.

Sedcole, N. P.; Blodget, B.; et al. (2006), "Modular dynamic reconfiguration in Virtex FPGAs". In IEE Proceedings-Computers and Digital Techniques, vol. 153, ed. 3, maio de 2006.

Sedcole, N. P. (2006), "Reconfigurable Platform-Based Design in FPGAs for Video Image Processing". Tese para obtenção de grau de Doutor, Universidade de Londres, janeiro de 2006.

Silva, J. L. (1992), "Processamento a Fluxo de Dados Tolerante a Falhas em um Computador Paralelo". Tese para obtenção de grau de Doutor, Universidade de Campinas, 1992.

Silva, J.L. (2006), "Execution of algorithms using a Dynamic Dataflow Model for Reconfigurable Hardware - A purpose for Matching Data". In the 6th International Workshop on System on Chip for Real Time Applications, pp. 115-119, 2006.

Silva, J.L.; Marques, E. (2006), "Executing algorithms for dynamic dataflow reconfigurable hardware - The operators protocol". In the 3rd International Conference on Reconfigurable Computing and FPGAs, pp. 1-7, 2006.

Silva, J.L.; Costa, K.A.P.; et al. (2009), "The C Compiler generating a Source file in VHDL for a Dynamic Dataflow Machine”. WSEAS Transactions on Computers, 2009.

Singh, H.; Ming-Hau Lee; Guangming Lu (2000), "MorphoSys: an integrated reconfigurable system for data-paralleland computation-intensive applications". In the IEEE Transactions on Computers, vol. 49, ed. 5, pp. 465-481, 2000.

Sirini, V. P. (1986), “An Architectural Comparison of Dataflow Systems”. In IEEE Computer, vol. 19 , n. 3, pp. 68-86, março de 1986.

Skliarova, I; Ferrari, A.B. (2003), "Introdução à Computação Reconfigurável”. Revista do DETUA, vol. 2, num. 6, setembro de 2003. 
Souza Jr., F.S. (2008), "ChipCflow - Validação e Implementação do Modelo de Partição e Protocolo de Comunicação no Grafo a Fluxo de Dados Dinâmico". Monografia apresentada para o Exame de Qualificação de Mestrado, Universidade de São Paulo, dezembro de 2008.

Spracklen, L.; Abraham, S.G. (2005), “Chip Multithreading: Opportunities and Challenges". In the 11th International Symposium on High-Performance Computer Architecture, pp. 248-252, 2005.

Swanson, S.; Michelson, K.; et al. (2003), "Wavescalar". In the 36th Annual International Symposium on Microarchitecture, pp. 291-302, 2003.

Swanson, S.; Putnam, A.; et al. (2006), "Area-performance trade-off in tiled dataflow architectures". In the International Symposium on Computer Architecture (ISCA), vol. 34, ed. 2, pp. 314-326, 2006.

Thoreson, S.A.; Long, A.N. (1987), “A Feasibility Study of Memory Hierarchy in Dataflow Environment". In the Proceedings of the International Conference on Parallel Processing, pp. 356-360, junho de 1987.

Tsu, W.; Macy, K.; Joshi, A.; et al. (1999), "HSRA: High-speed, Hierarchical Synchronous Reconfigurable Array". In ACM/SIGDA International Symposium on Field-Programmable Gate Arrays, pp. 125-134, 1999.

Tuan, T.; Lai, B. (2003), "Leakage Power Analysis of a 90nm FPGA". In IEEE 2003 Custom Integrated Circuits Conference, pp. 57-60, 2003.

Veen A.H. (1980), "The Misconstrued Semicolon: Reconciling Imperative Languages and Dataflow Machines”. Mathematisch Centrum, ISBN 90-6196-302-8, 1980.

Veen, A. H. (1986), "Dataflow machine architecture". In the ACM Computing Surveys, vol. 18, ed. 4, pp. 365-396, dezembro de 1986. 
Vuillemin, J.E.; Bertin, D.; et al. (1996), "Programmable Active Memories: Reconfigurable Systems Come of Age". In the IEEE Transactions on Very Large Scale Integration (VLSI) Systems, vol. 4, ed. 1, pp. 56-69, março de 1996.

Wang, H.; Delahaye, J.P.; et al. (2007), "Managing dynamic reconfiguration on MIMO Decoder”. Parallel and Distributed Processing Symposium, pp. 1-8, março de 2007.

Wilson, R. (2008), "The FPGA industry strives to keep on scaling”. Eletronics Design, Strategy, News website: http://www.edn.com/article/CA6569196.html <Acesso em setembro de 2009>.

Wirthlin, M.J.; Hutchings, B.L (1995), “A dynamic instruction set computer”. In the IEEE Symposium on FPGA's for Custom Computing Machines (FCCM '95), pp. 99-107, abril de 1995.

Wolfe, A. (2008), “Intel Blog Warns Of Multicore Crisis”. The InformationWeek, 2008.

Xilinx, Inc, URL a: http://www.xilinx.com <Acesso em setembro de 2009>.

Xilinx, Inc, URL b. Partial Reconfiguration Early Access Tools Lounge: http://www.xilinx.com/support/prealounge/protected/index.htm <Acesso em setembro de 2009>.

Xilinx (2005), “Development System Reference Guide”. Xilinx Inc., 2005.

Xilinx (2006a), "Early Access Partial Reconfiguration User Guide For ISE 8.1.01i”. Xilinx Inc, 2006.

Xilinx (2007a), "Virtex-II Platform FPGAs: Complete Data Sheet". Xilinx Inc., Xilinx Data Sheet 031, v3.5, novembro de 2007.

Xilinx (2007b), “Virtex-4 User Guide”. Xilinx Inc., Xilinx User Guide 070, v2.3, agosto de 2007.

Xilinx (2007c), "Virtex-5 Family Overview LX, LXT, and SXT Platforms". Xilinx Inc., Xilinx Data Sheet 100, v3.4, dezembro de 2007. 
Xilinx (2007d), “Xilinx ISE 9.2i Software Manuals and Help". Xilinx Inc., Xilinx PDF Collection, 2007.

Xilinx (2007e), "Difference-Based Partial Reconfiguration". Xilinx, Inc, Xilinx Application Note 290, v2.0, dezembro de 2007.

Xilinx (2007f), "Partial Reconfiguration Design with PlanAhead 9.2". Xilinx, Inc, dezembro de 2007.

Xilinx (2007g), "Partial Reconfiguration Flow Design Examples Using ISE 9.1.2 with PR10 Overlay Targeting ML403 Board”. Xilinx, Inc, agosto de 2007.

Xilinx (2007h), "Partial Reconfiguration Flow Design Examples Using ISE 9.1.2 with PR10 Overlay Targeting XUPV2P Board". Xilinx, Inc, setembro de 2007.

Xilinx (2008a), "PlanAhead Tutorial". Xilinx, Inc, 2008.

Xilinx (2008b), "Partial Reconfiguration Design with PlanAhead, Version 2.1". Xilinx, Inc, março de 2008.

Xilinx (2008c), "Early Access Partial Reconfiguration User Guide For ISE 9.2.04i". Xilinx, Inc, setembro de 2008. 


\section{Apêndice A}

\section{Código-fonte do operador OPADD Dinâmico, OPADD_D.}

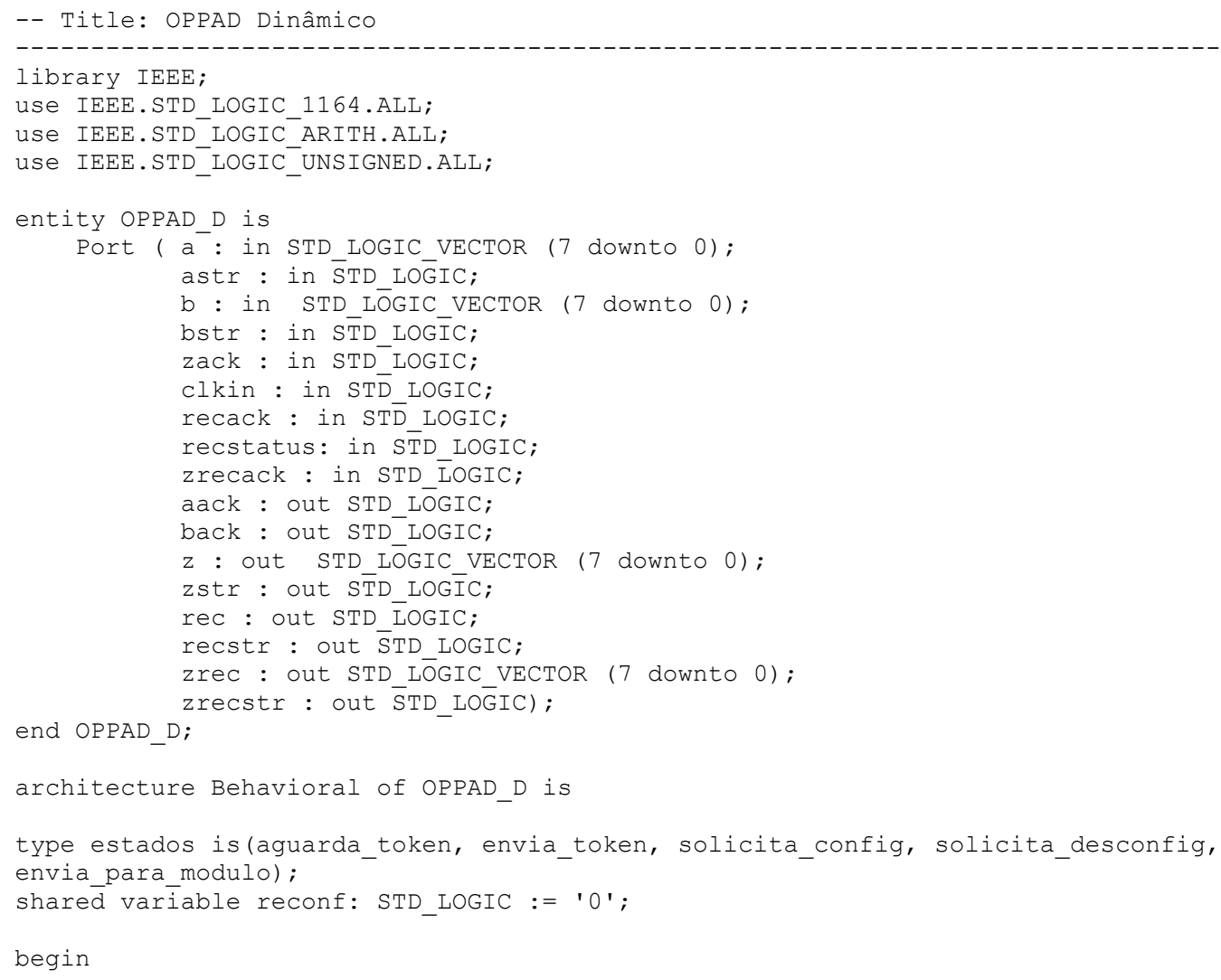




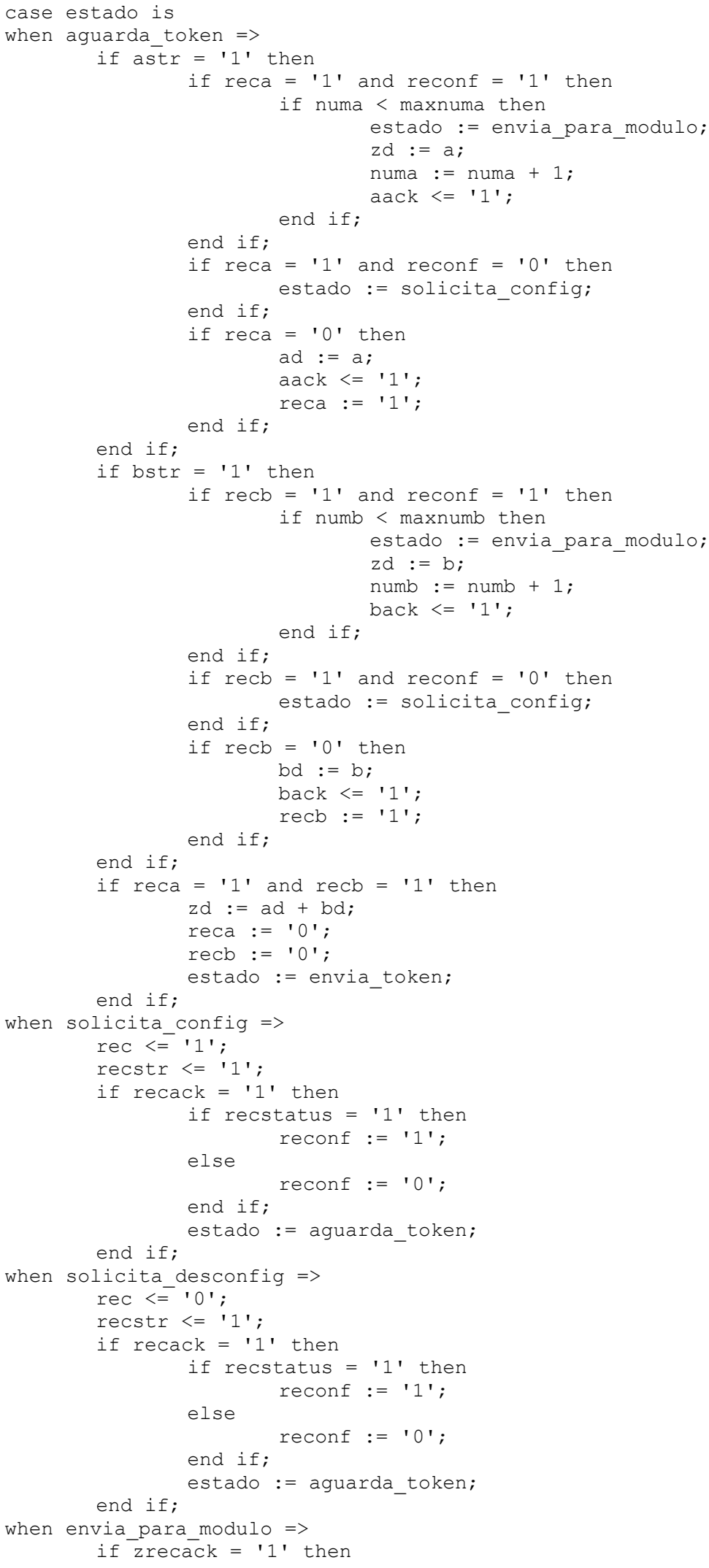




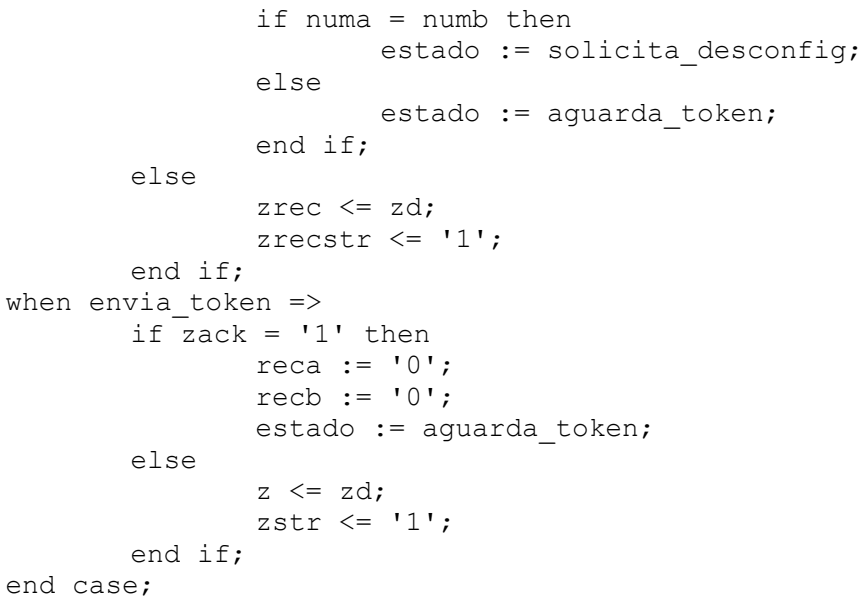




\section{Apêndice B}

\section{Código-fonte do módulo OPADD_R.}

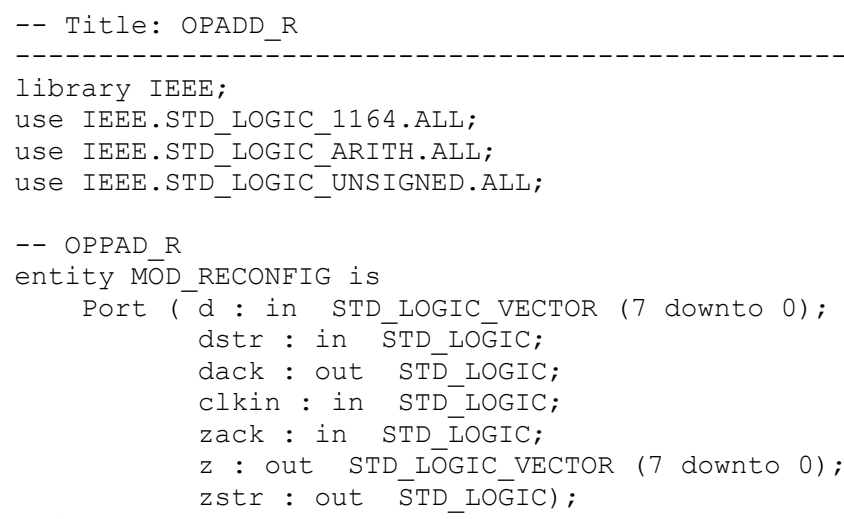




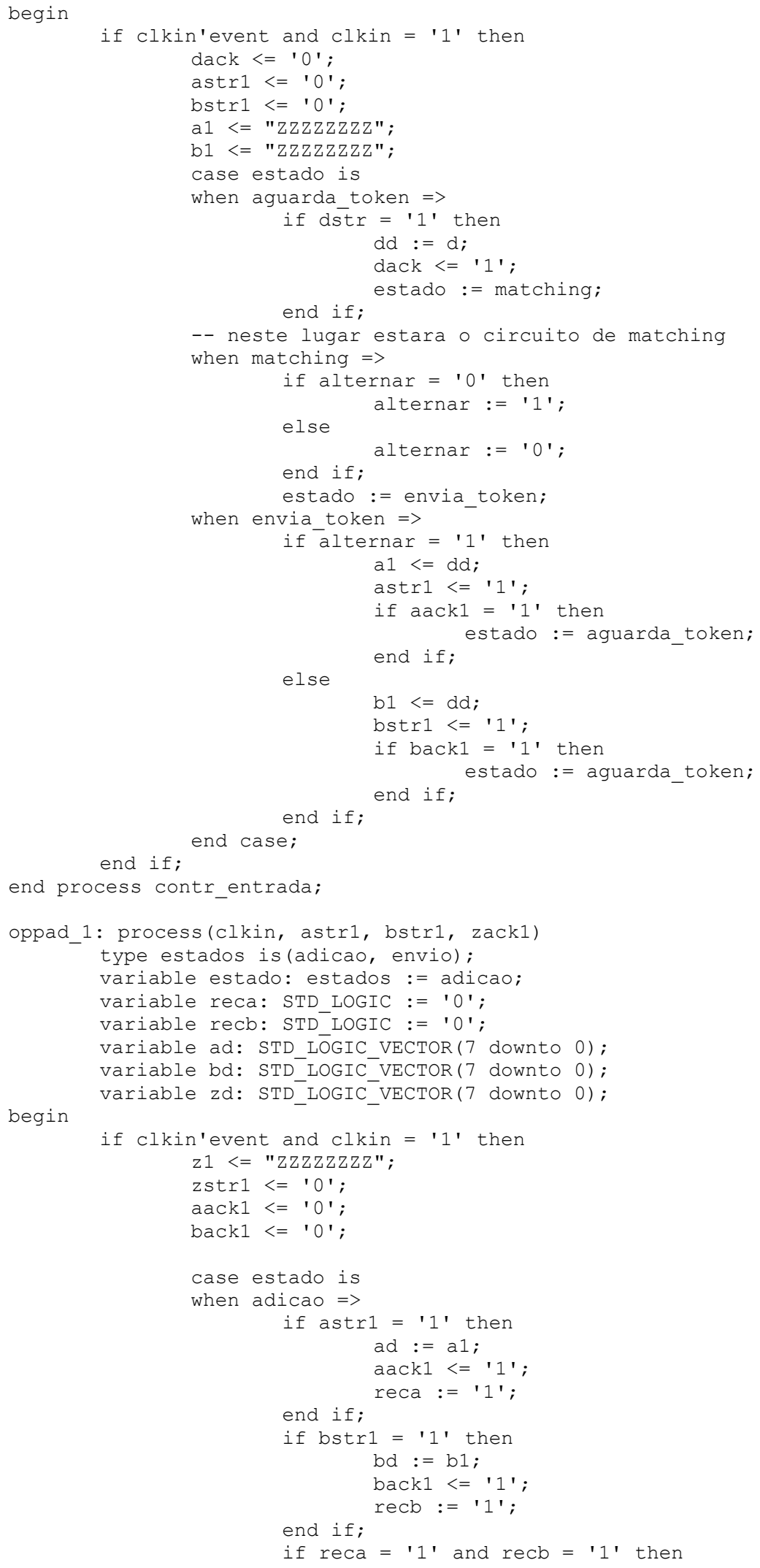




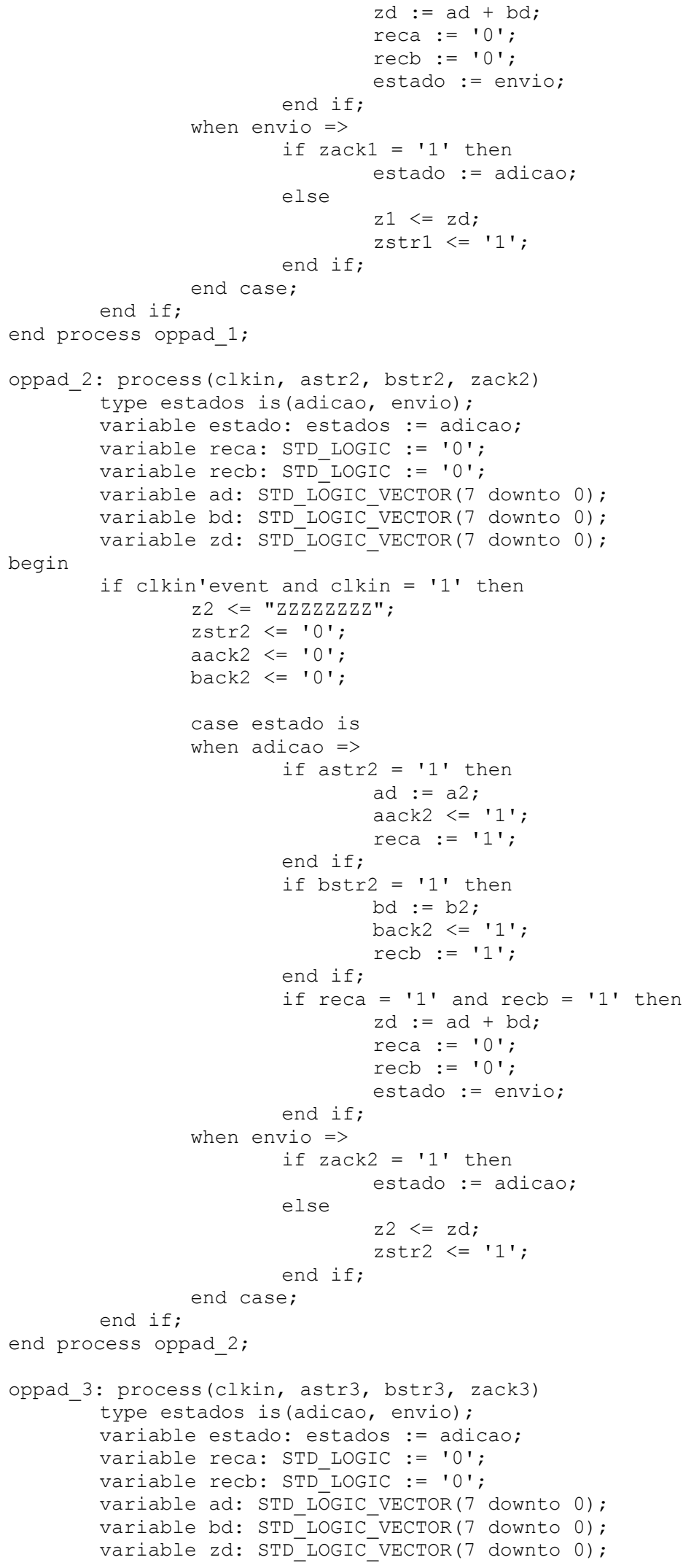




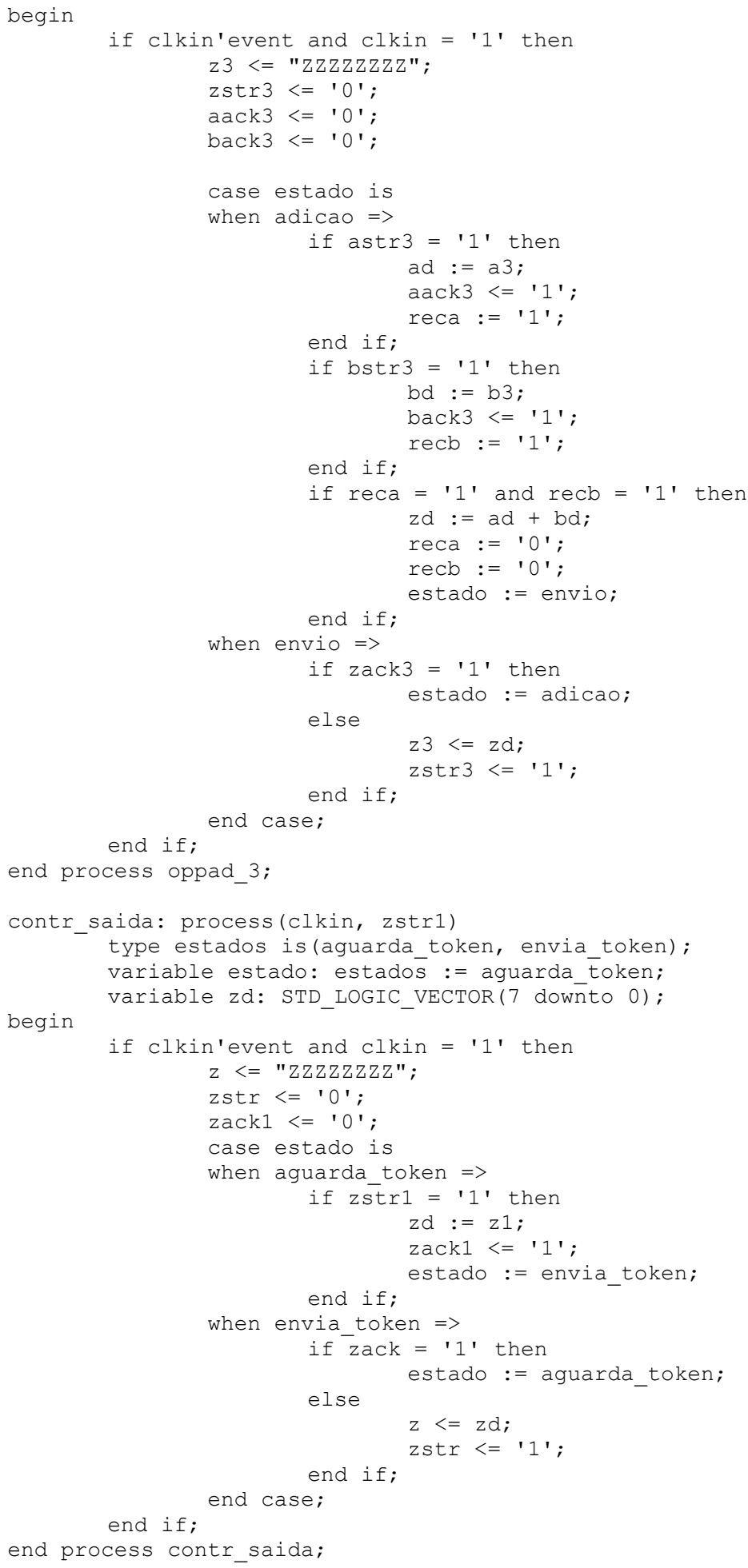




\section{Apêndice C}

Código-fonte do arquivo de topo do grafo implementado como prova de conceito para o modelo proposto.

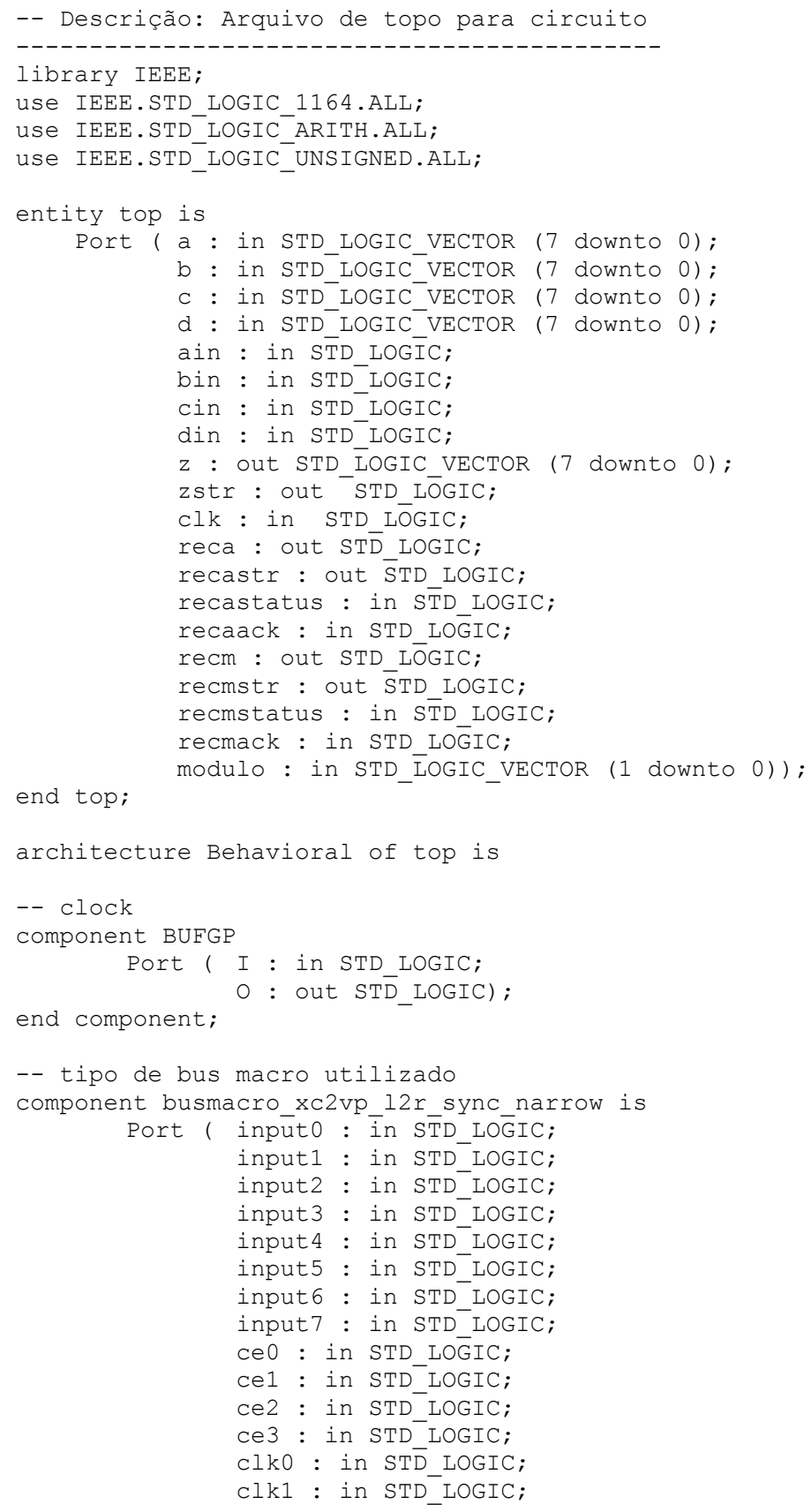




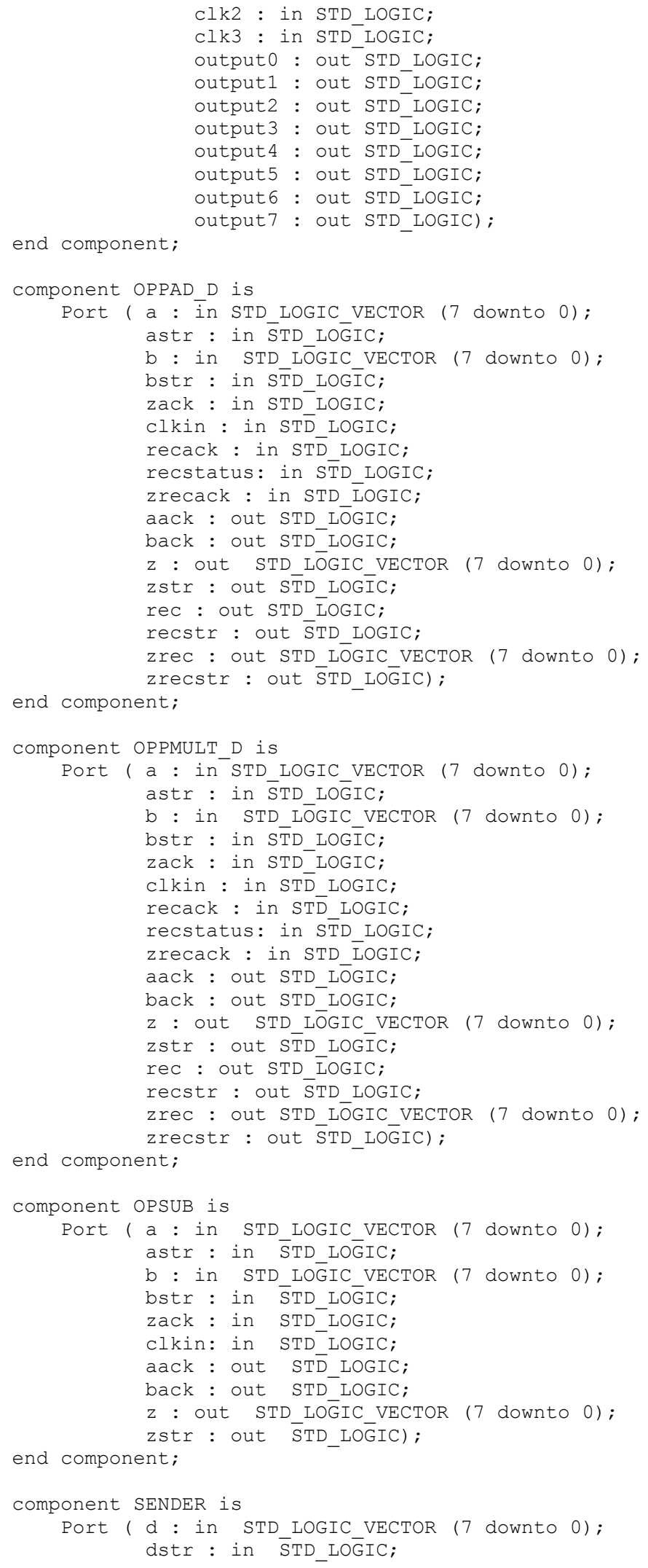




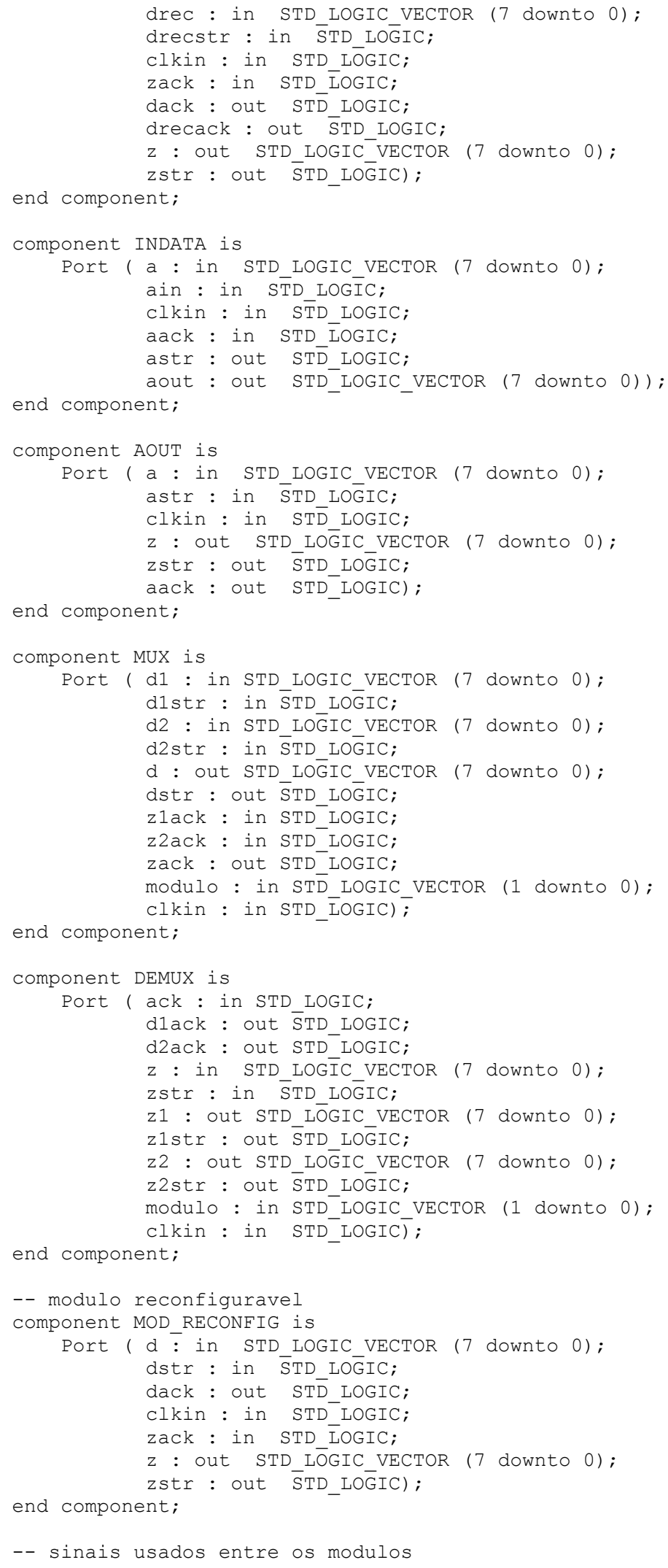




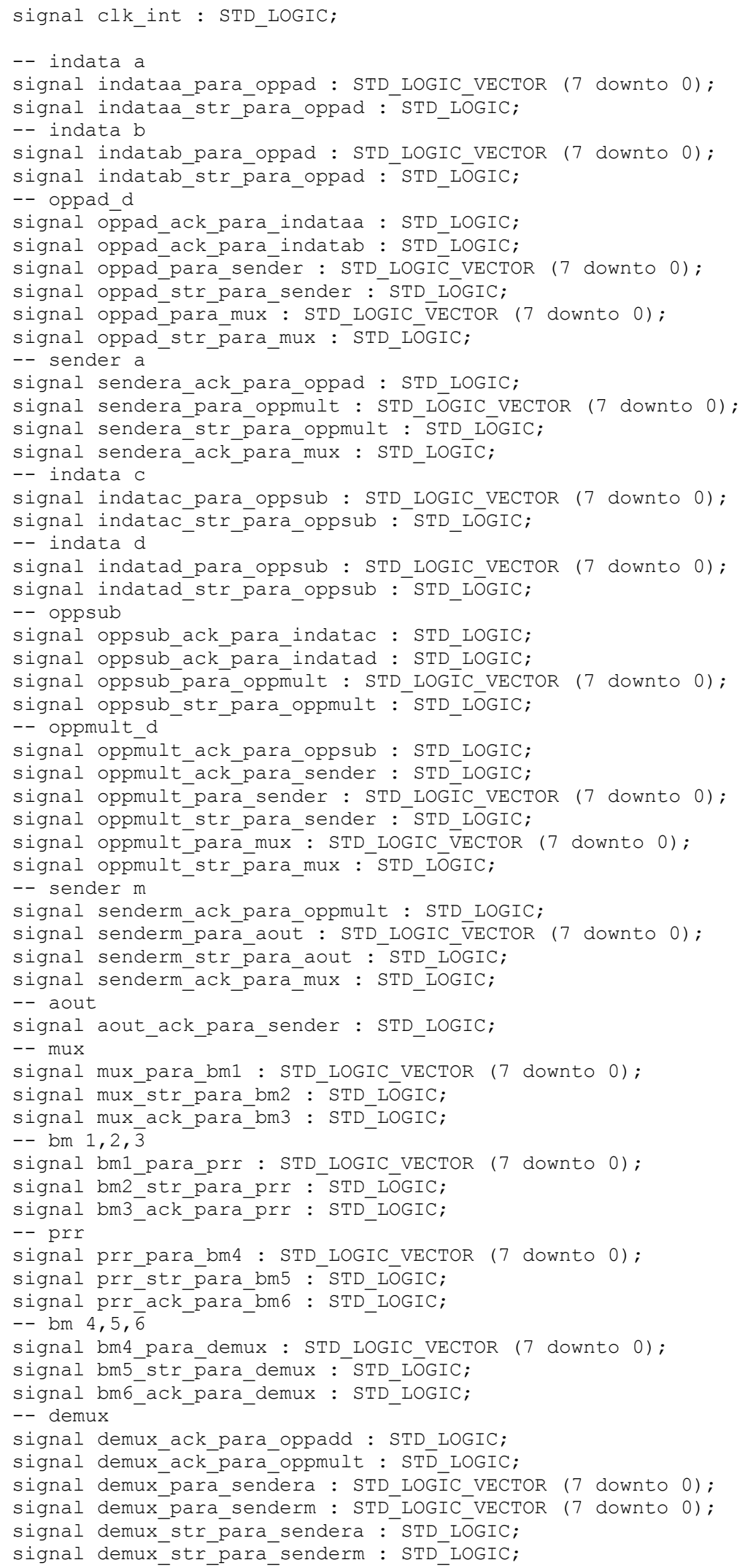




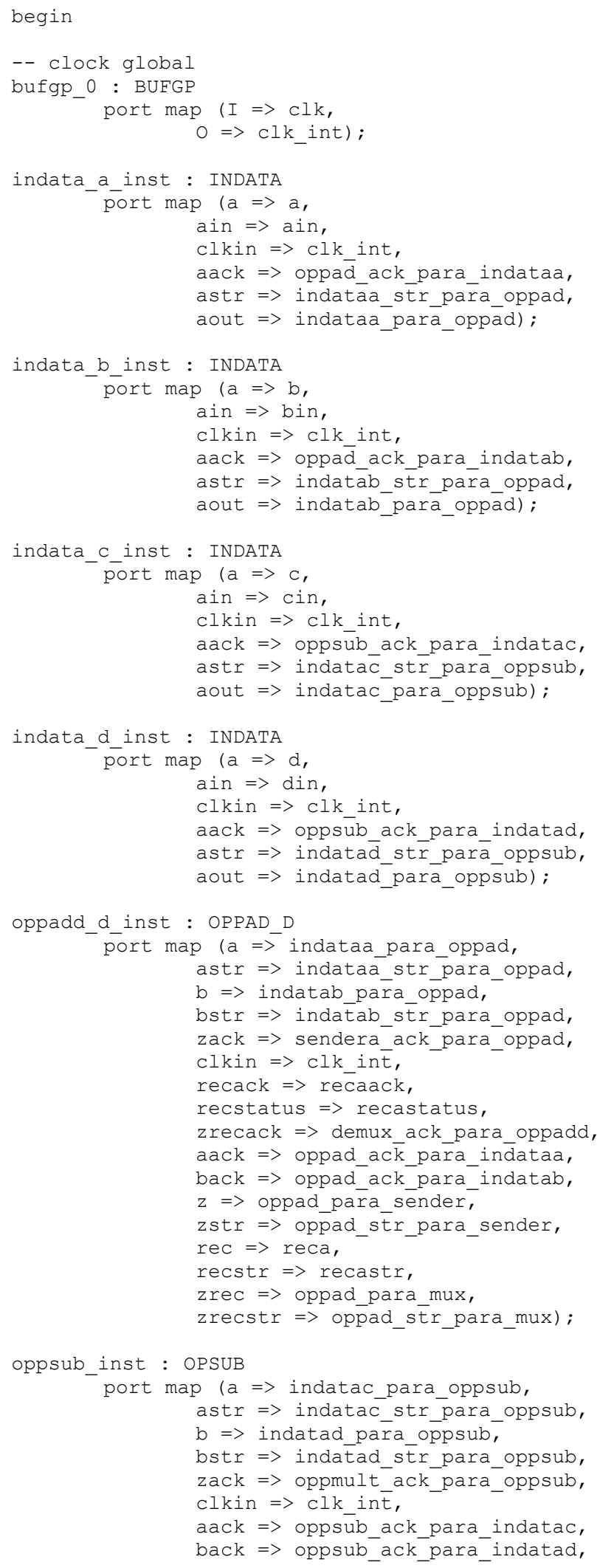




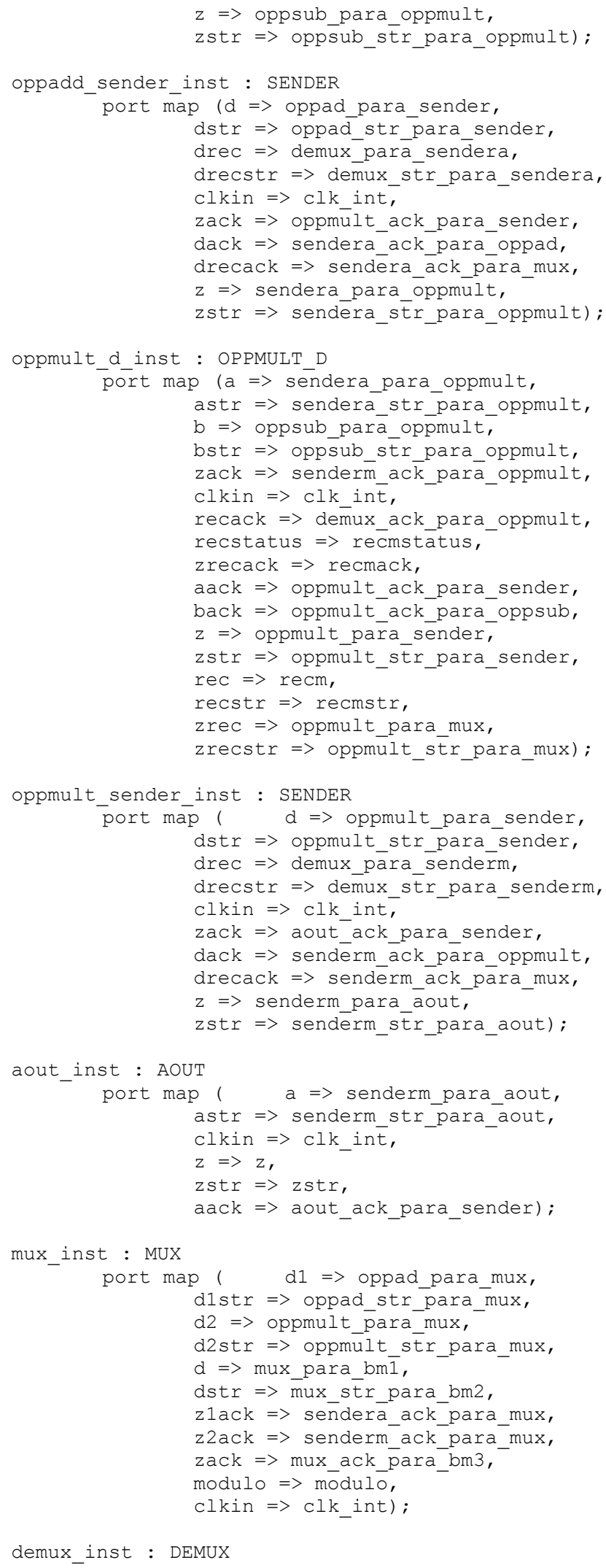




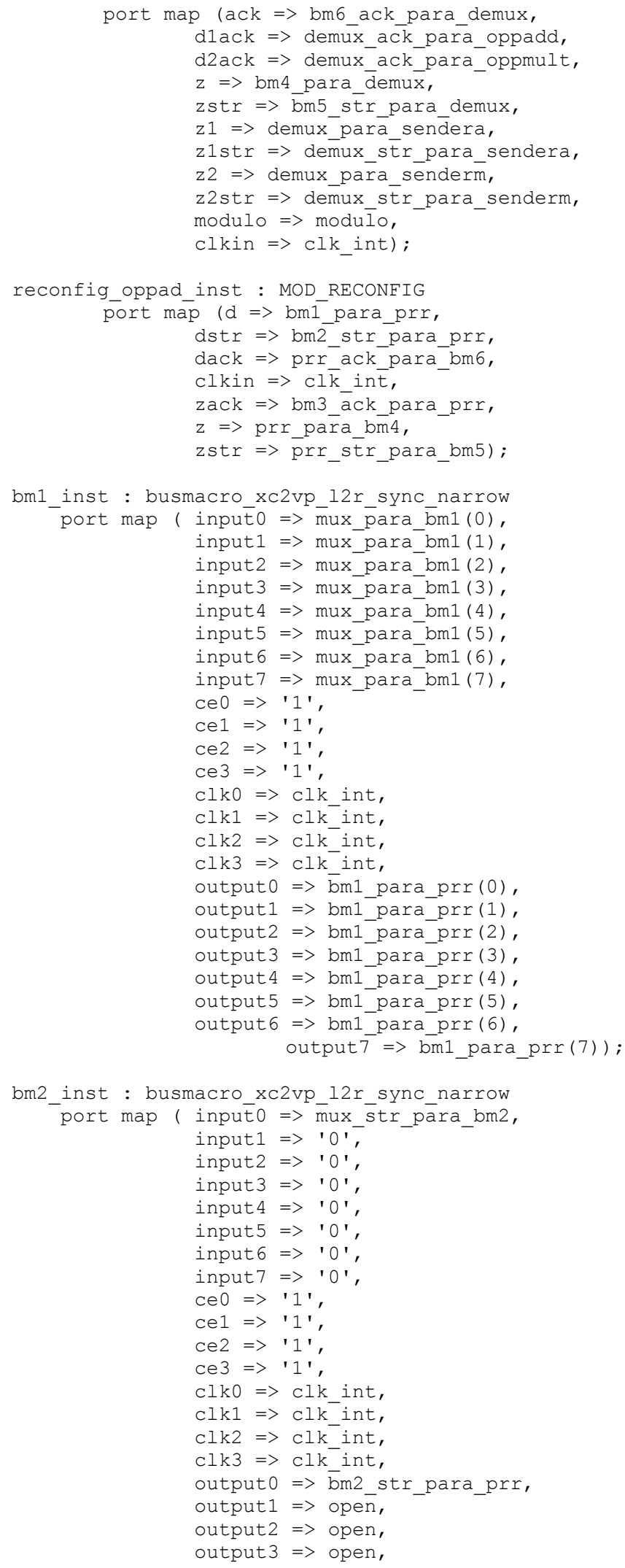




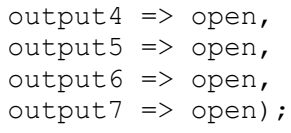

bm3_inst : busmacro_xc2vp_12r_sync_narrow port map ( input0 $\Rightarrow$ mux ack para bm3,

input1 $=>$ '0',

input2 $=>$ '0',

input3 $=>$ '0',

input $4 \Rightarrow>$ ',

input5 $=>$ '0',

input $6=>$ '0',

input $7=>$ '0',

ce0 $=>$ ' 1 ',

cel $=>$ ' 1 ',

ce2 $=>$ ' 1 ',

ce3 $=>1$ ' ',

clk0 $=>$ clk_int,

clk1 $\Rightarrow$ clkint,

clk2 =>clkint,

clk3 $=>$ clkint,

output0 => bm3_ack_para_prr,

output $1=>$ open,

output $2=>$ open,

output $3=>$ open,

output $4=>$ open,

output $5=>$ open,

output $6=>$ open,

output $7=>$ open);

bm4_inst : busmacro_xc2vp_12r_sync narrow

port map ( inputo $\Rightarrow$ prr_para bm4 (0),

input1 $=>$ prr para bm4 (1),

input2 $=>$ prr_para bm4 (2),

input $3=>$ prr para bm4 (3)

input4 4 prr_para_bm4 (4),

input $5=>$ prr para bm4 (5),

input6 $=>$ prr_para_bm4 (6),

input $7 \Rightarrow$ pre para bm4 (7),

ce0 $=>$ ' 1 ',

cel $1=>$ ' 1 '

ce2 $=>1^{\prime}$,

ce3 $=>$ ' 1 ',

clk0 $=>$ clk int,

clkl $=>$ clkint,

clk2 $\Rightarrow$ clkint,

clk3 $=>$ clk-int,

output $0=>$ bm 4 para demux $(0)$

output1 => bm4_para_demux(1),

output2 => bm4 para demux(2),

output3 3 bm4_para_demux (3),

output4 $4>$ bm4 para demux (4),

output $5=>$ bm4_para_demux (5),

output $6=>$ bm 4 para demux (6),

output 7 = bm4 para_demux(7));

bm5 inst : busmacro xc2vp 12 r sync narrow

port map ( inputo $=>$ prr_ster_para_bm5, input $1 \Rightarrow$ ' 0 ',

input2 $=>$ '0', input $3=>$ ' 0 ', input $4=>$ '0', input $5=>$ '0', input $6 \Rightarrow$ '0', input $7=>$ '0', ce0 $=>$ ' 1 ',

cel $1=>1^{\prime}$, 


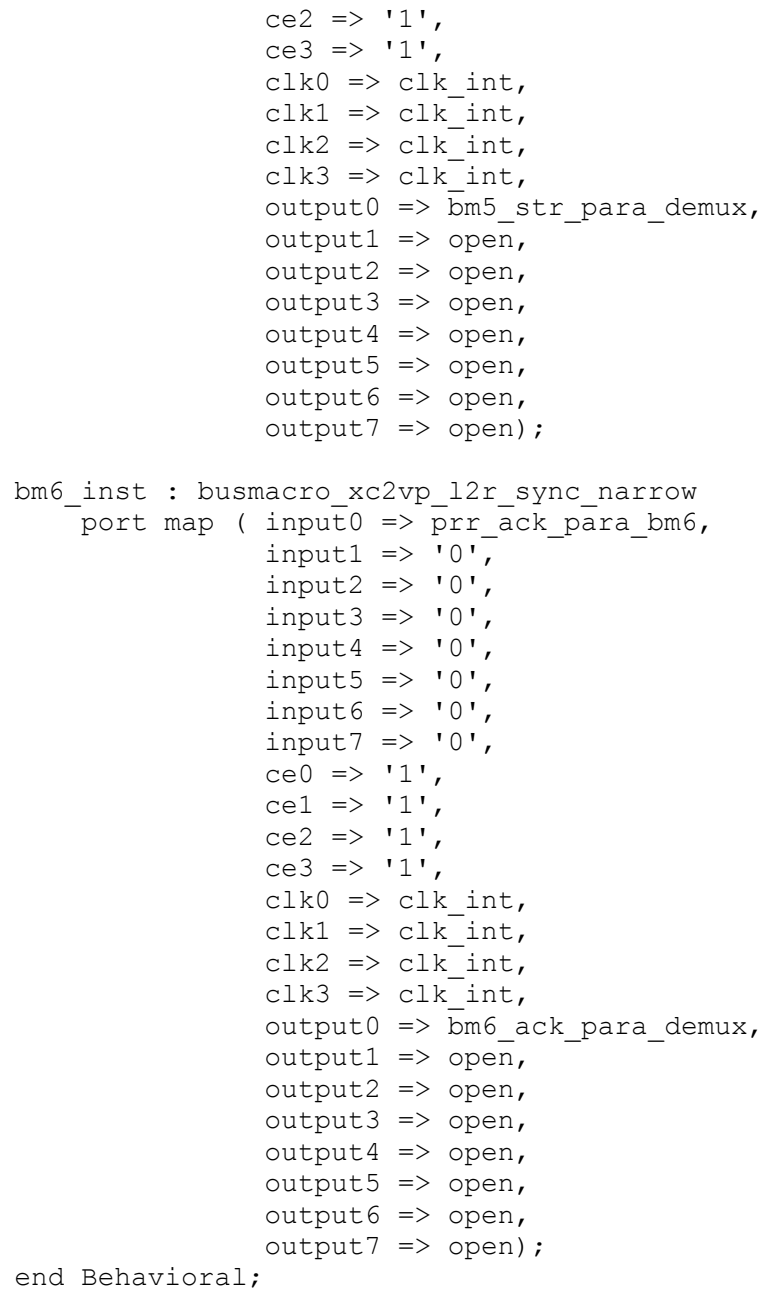

